\title{
Constraints on the production and escape of ionizing radiation from the emission-line spectra of metal-poor star-forming galaxies
}

\author{
A. Plat,${ }^{1} *$ S. Charlot,${ }^{1}$ G. Bruzual, ${ }^{2}$ A. Feltre,${ }^{1,3,4}$ A. Vidal-García, ${ }^{1,5}$ C. Morisset,${ }^{6}$ \\ J. Chevallard ${ }^{1}$ and H. Todt ${ }^{7}$ \\ ${ }^{1}$ Sorbonne Université, CNRS, UMR7095, Institut d'Astrophysique de Paris, F-75014, Paris, France \\ ${ }^{2}$ Instituto de Radioastronomía y Astrofísica, UNAM, Campus Morelia, Michoacan, México, C.P. 58089, México \\ ${ }^{3}$ SISSA, via Bonomea 265, I-34136 Trieste, Italy \\ ${ }^{4}$ Univ. Lyon, Univ. Lyon1, ENS de Lyon, CNRS, Centre de Recherche Astrophysique de Lyon, UMR5574, 69230 Saint-Genis-Laval, France \\ ${ }^{5}$ LERMA, Observatoire de Paris, Ecole Normale Supérieure, PSL Research University, CNRS, UMR8112, F-75014 Paris, France \\ ${ }^{6}$ Instituto de Astronomía, UNAM, Apdo. postal 106, C.P. 22800 Ensenada, Baja California, México \\ ${ }^{7}$ Institute of Physics and Astronomy, University of Potsdam, Karl-Liebknecht-Str. 24/25, 14476 Potsdam, Germany
}

Accepted 2019 September 13. Received 2019 September 9; in original form 2019 July 12

\begin{abstract}
We explore the production and escape of ionizing photons in young galaxies by investigating the ultraviolet and optical emission-line properties of models of ionizationbounded and density-bounded HII regions, active-galactic-nucleus (AGN) narrow-line regions and radiative shocks computed all using the same physically-consistent description of element abundances and depletion on to dust grains down to very low metallicities. We compare these models with a reference sample of metal-poor star-forming galaxies and Lyman-continuum (LyC) leakers at various redshifts, which allows the simultaneous exploration of more spectral diagnostics than typically available at once for individual subsamples. We confirm that current single- and binary-star population synthesis models do not produce hard-enough radiation to account for the high-ionization emission of the most metal-poor galaxies. Introducing either an AGN or radiative-shock component brings models into agreement with observations. A published model including X-ray binaries is an attractive alternative to reproduce the observed rise in $\mathrm{He}$ II $\lambda 4686 / \mathrm{H} \beta$ ratio with decreasing oxygen abundance in metal-poor star-forming galaxies, but not the high observed $\mathrm{He}$ II $\lambda 4686 / \mathrm{H} \beta$ ratios of galaxies with large $\mathrm{EW}(\mathrm{H} \beta)$. A source of harder ionizing radiation appears to be required in these extreme objects, such as an AGN or radiative-shock component, perhaps linked to an initial-mass-function bias toward massive stars at low metallicity. This would also account for the surprisingly high [O I]/[O III] ratios of confirmed LyC leakers relative to ionization-bounded models. We find no simple by-eye diagnostic of the nature of ionizing sources and the escape of LyC photon, which require proper simultaneous fits of several lines to be discriminated against.
\end{abstract}

Key words: galaxies: general - galaxies: high-redshift - galaxies: ISM.

\section{INTRODUCTION}

The nebular emission from primeval galaxies represents one of our best hopes to constrain the physical processes that dominated reionization of our Universe. Beyond the physical conditions of pristine gas, emission lines are sensitive to different components expected to characterise primeval galaxies: hot massive stars, often considered as the main source of ionizing radiation; active

*E-mail: plat@iap.fr galactic nuclei (AGN), arising from gas accretion onto primordial black holes; radiative shocks, induced by large-scale gas flows; and the leakage of Lyman-continuum (LyC) photons through a porous interstellar medium (ISM), contributing to reionization (see, e.g., the review by Stark 2016). In waiting for advent of the James Webb Space Telescope (JWST), which will enable deep rest-frame ultraviolet and optical emission-line spectroscopy of galaxies into the reionization era at redshifts $z \sim 10$ 15 , more nearby metal-poor galaxies approaching the properties of primeval galaxies offer a useful laboratory in which to test our ability to interpret emission-line spectra. 


\section{A.Plat et al.}

Observationally, a fast-growing number of studies have progressively uncovered the spectroscopic properties of distant, metal-poor star-forming galaxies at redshifts out to $z \gtrsim 7$ (e.g., Erb et al. 2010; Stark et al. 2014, 2015; Rigby et al. 2015; Amorín et al. 2017; Laporte et al. 2017; Schmidt et al. 2017; Mainali et al. 2017; Vanzella et al. 2017; Berg et al. 2018; Nakajima et al. 2018; Nanayakkara et al. 2019; Tang et al. 2019) and in parallel those of nearby analogues of these pristine galaxies (e.g., Berg et al. 2016, 2019; Senchyna et al. 2017, 2019). Most of these studies focused on identifying promising tracers and diagnostics of the early chemical enrichment and gas conditions in primeval galaxies, such as for example the He II $\lambda 1640, \mathrm{C}$ III] $\lambda 1908$ and C IV $\lambda 1549$ lines and C/O abundance ratio. Other studies were more specifically aimed at probing LyC-photon leakage from young star-forming galaxies, using clues such as a small velocity spread of the Ly $\alpha$ double-peaked emission or large ratios of highto low-ionization lines (e.g., Jaskot \& Oey 2013; Izotov et al. 2016a, 2017, 2018b; de Barros et al. 2016; Vanzella et al. 2016). Meanwhile, on the theoretical front, much effort was invested into characterising the ionizing spectra and ultraviolet emissionline signatures of young stellar populations (e.g., Gutkin et al. 2016; Vidal-García et al. 2017; Byler et al. 2018), along with the dependence of these on stellar rotation and binary interactions (e.g., Levesque \& Leitherer 2013; Stanway \& Eldridge 2019), as well as the signatures of active galactic nuclei (AGN; e.g., Feltre et al. 2016; Hirschmann et al. 2017, 2019; Nakajima et al. 2018) and shock-ionized gas (e.g., Allen et al. 2008; Izotov et al. 2012; Alarie \& Morisset 2019).

In practice, the observational studies mentioned above provide a valuable set of, in some respects, independent investigations focusing individually on the analysis of a specific set of emission lines with specific models (for the production of radiation and the photoionization calculations), parametrized in a specific way (e.g., element abundances and depletions; inclusion or not of dust physics in the photoionization calculations), depending on the nature and redshift of the sample and the spectrograph employed. These analyses have brought important lessons, such as the usefulness of the C III] $\lambda 1908$ and even C IV $\lambda 1549$ lines as signposts of galaxies in the reionization era given the expected strong attenuation of $\operatorname{Ly} \alpha$ (e.g., Stark et al. 2014; Senchyna et al. 2019 ) and the potentiality of the CIV $\lambda 1549 / \mathrm{He}$ II $\lambda 1640$ luminosity ratio for identifying AGN (e.g., Feltre et al. 2016; Nakajima et al. 2018), the C III] $\lambda 1908 / \mathrm{He}$ II $\lambda 1640$ ratio for identifying shock-ionized gas (Jaskot \& Ravindranath 2016) and the [O III] $\lambda 5007 /[\mathrm{O}$ II] $] \lambda 3727$ ratio for identifying LyC-photon leakage (e.g., Nakajima \& Ouchi 2014; Izotov et al. 2016a).

A current difficulty in reaching a robust picture from this progress on several fronts in parallel is that the conclusions drawn from fitting a restricted set of emission lines using specific models may not be consistent with findings based on other lines and different models. This may be particularly important, for example, in the context of interpreting the exceedingly large strengths of He II recombination lines (requiring photon energies $E_{\mathrm{ion}}>54.4 \mathrm{eV}$ ) found in very metal-poor, actively star-forming galaxies, which seem to elude standard model predictions (e.g., Shirazi \& Brinchmann 2012; Senchyna et al. 2017; Berg et al. 2018; Nanayakkara et al. 2019; Stanway \& Eldridge 2019). Based on various arguments, contributions from very massive stars (e.g., Gräfener \& Vink 2015), stripped stars produced by close-binary evolution or X-ray binaries (e.g., Senchyna \& Stark 2019; Schaerer et al.
2019), AGN (e.g., Nakajima et al. 2018) and radiative shocks (e.g., Izotov et al. 2012) have been proposed to account for the required hard radiation. Also, while the [O III] $\lambda 5007 /[\mathrm{O}$ II $] \lambda 3727$ diagnostic to characterise galaxies leaking LyC photons may not be as reliable as expected and alternative diagnostics based, e.g., on He I lines have been suggested, different types of investigations of the ultraviolet and optical signatures of LyC leakage have so far focused on rather limited sets of emission lines (e.g., Jaskot \& Oey 2013; Zackrisson et al. 2013, 2017; Stasińska et al. 2015; Jaskot \& Ravindranath 2016; Izotov et al. 2017). These examples illustrate the need for a homogeneous investigation of emission-line diagnostics of metal-poor star-forming galaxies with a wide collection of intercomparable models (of the type of that proposed by Stasińska et al. 2015 for a few optical lines).

In this paper, we examine a full set of ultraviolet/optical observables of metal-poor star-forming galaxies with a library of nebular-emission models enabling the exploration of a wide range of physical parameters. To conduct this analysis, we build a reference sample of ultraviolet and optical observations of metal-poor star-forming galaxies and confirmed and candidate LyC leakers (and other star-forming galaxies and AGN) in a wide redshift range. This sample allows us to simultaneously explore diagnostic diagrams involving more emission lines than typically available at once for individual subsamples. We use this sample to investigate potentially discriminating signatures of singleand binary-star populations (using the most recent versions of the Bruzual \& Charlot 2003 and Eldridge et al. 2017 models), narrow-line regions of AGN (Feltre et al. 2016) and radiative shocks (Alarie \& Morisset 2019) on the emission-line properties of metal-poor star-forming galaxies, adopting throughout the same parametrization of nebular-gas abundances (Gutkin et al. 2016).

Our analysis confirms that current single- and binary-star population synthesis models do not produce hard-enough ionizing radiation to account for the strong He II emission seen in some of the most metal-poor galaxies, although with slightly better agreement than concluded recently by Stanway \& Eldridge (2019). We show that an AGN or radiative-shock component allows models to reproduce observations in nearly all the ultraviolet and optical line-ratio diagrams we investigate. We also consider X-ray binaries as a potential source of ionizing radiation, using the model recently proposed by Schaerer et al. (2019). This can reproduce the observed rise in $\mathrm{He}$ II $\lambda 4686 / \mathrm{H} \beta$ ratio toward low metallicities in star-forming galaxies, but not the high observed $\mathrm{He}$ II $\lambda 4686 / \mathrm{H} \beta$ ratios of galaxies with large $\mathrm{EW}(\mathrm{H} \beta)$. A source of harder ionizing radiation appears to be required in these extreme objects. In the end, we find that while none of the ultraviolet and optical emission-line diagrams we consider allows simple by-eye diagnostics of the nature of ionizing sources and the escape of $\mathrm{LyC}$ photons in metal-poor star-forming galaxies, differences exist in the spectral signatures of these physical quantities, which should enable more stringent constraints from simultaneous fits of several lines using tools such as BEAGLE (Chevallard \& Charlot 2016).

We present our models of ionization-bounded and densitybounded galaxies, AGN narrow-line regions and radiative shocks in Section 2. In Section 3, we assemble the reference sample of metal-poor star-forming galaxies, LyC leakers and other starforming galaxies and AGN, which we use in Section 4 to explore 
the influence of the different adjustable parameters of the models on emission-line spectra. In Section 5, we investigate potentially discriminating emission-line diagnostics of the production and escape of ionizing radiation in metal-poor star-forming galaxies. Our conclusions are summarized in Section 6.

\section{MODELLING APPROACH}

In this section, we present the set of versatile models that will be used in Section 4 to explore, in a physically consistent way, the influence of a wide range of parameters on the observed ultraviolet and optical nebular emission from young star-forming galaxies. We start by describing the models we adopt to compute properties of ionization-bounded galaxies. Then, we describe our approach to model density-bounded galaxies. We also appeal to existing prescriptions to include the contributions by AGN and shock-ionized gas to the nebular emission from galaxies.

\subsection{Ionisation-bounded models}

We adopt the approach introduced by Charlot \& Longhetti (2001, see also Gutkin et al. 2016) to compute the nebular emission from ionization-bounded galaxies. This is based on the combination of a stellar population synthesis model with a photoionization code to compute the luminosity per unit wavelength $\lambda$ emitted at time $t$ by a star-forming galaxy as

$L_{\lambda}(t)=\int_{0}^{t} \mathrm{~d} t^{\prime} \psi\left(t-t^{\prime}\right) S_{\lambda}\left[t^{\prime}, Z\left(t-t^{\prime}\right)\right] T_{\lambda}\left(t, t^{\prime}\right)$,

where $\psi\left(t-t^{\prime}\right)$ is the star formation rate at time $t-t^{\prime}, S_{\lambda}\left[t^{\prime}, Z(t-\right.$ $\left.\left.t^{\prime}\right)\right]$ the luminosity produced per unit wavelength per unit mass by a single stellar generation of age $t^{\prime}$ and metallicity $Z\left(t-t^{\prime}\right)$ and $T_{\lambda}\left(t, t^{\prime}\right)$ the transmission function of the ISM. Following Charlot \& Fall (2000, see also Vidal-García et al. 2017), we write

$T_{\lambda}\left(t, t^{\prime}\right)=T_{\lambda}^{\mathrm{BC}}\left(t^{\prime}\right) T_{\lambda}^{\mathrm{ICM}}(t)$.

where $T_{\lambda}^{\mathrm{BC}}\left(t^{\prime}\right)$ is the transmission function of stellar birth clouds (i.e. giant molecular clouds) and $T_{\lambda}^{\mathrm{ICM}}(t)$ that of the intercloud medium (i.e. diffuse ambient ISM). In the present study, we focus on young galaxies with ages close to the typical timescale for the dissipation of giant molecular clouds in star-forming galaxies ( 10 Myr, ; e.g., Murray et al. 2010; Murray 2011) and do not include any intercloud medium. The birth clouds, assumed all identical, are described as an inner HıI region ionized by young stars and bounded by an outer HI region (Charlot \& Fall 2000). We thus write

$T_{\lambda}\left(t, t^{\prime}\right)=T_{\lambda}^{\mathrm{BC}}\left(t^{\prime}\right)=T_{\lambda}^{\mathrm{HII}}\left(t^{\prime}\right) T_{\lambda}^{\mathrm{HI}}\left(t^{\prime}\right)$.

By analogy with Charlot \& Longhetti (2001, see also Gutkin et al. 2016), we compute the transmission function $T_{\lambda}^{\mathrm{HII}}\left(t^{\prime}\right)$ of the ionized gas $\left[T_{\lambda}^{+}\left(t^{\prime}\right)\right.$ in their notation] using the photoionization code CLOUDY (we adopt here version c17.00; Ferland et al. 2017). In this approach, the galaxy-wide transfer of stellar radiation through ionized gas is described via a set of 'effective' parameters. The main adjustable parameters are (see Gutkin et al 2016 for details): (i) The (hydrogen) gas density, $n_{\mathrm{H}}$.

(ii) The total gas metallicity, assumed to be equal to that of the ionizing stars, $Z$. We adopt the chemical-element abundances listed in table 1 of Gutkin et al. (2016), ${ }^{1}$ corresponding to a present-day solar (photospheric) metallicity $Z_{\odot}=0.01524$ and a protosolar metallicity (i.e. before the effects of diffusion) $Z_{\odot}^{0}=0.01774$. Nitrogen and carbon are assumed to both have primary and secondary nucleosynthetic components. The total (primary+secondary) nitrogen abundance is related to that of oxygen via equation (11) of Gutkin et al. (2016).

(iii) The carbon-to-oxygen abundance ratio, C/O. This adjustable parameter allows secondary $\mathrm{C}$ production to be kept flexible [for reference, $\left.(\mathrm{C} / \mathrm{O})_{\odot}=0.44\right]$.

(iv) The dust-to-metal mass ratio, $\xi_{\mathrm{d}}$, which reflects the depletion of heavy elements on to dust grains $\left(\xi_{\mathrm{d} \odot}=0.36\right.$; see table 1 of Gutkin et al. 2016).

(v) The volume-averaged ionisation parameter at age $t^{\prime}=0$, noted simply $\langle U\rangle \equiv\langle U\rangle\left(t^{\prime}=0\right)$. The volume-averaged ionisation parameter of a spherical Hi region can be expressed as (e.g., equation 3 of Panuzzo et al. 2003)

$\langle U\rangle\left(t^{\prime}\right)=\frac{3 \alpha_{B}^{2 / 3}}{4 c}\left[\frac{3 Q\left(t^{\prime}\right) \epsilon^{2} n_{\mathrm{H}}}{4 \pi}\right]^{1 / 3}$,

where $Q\left(t^{\prime}\right)$ is the time-dependent rate of ionizing photons produced by a single stellar generation of age $t^{\prime}, \epsilon$ the volume-filling factor of the gas (i.e., the ratio of the volume-averaged hydrogen density to $\left.n_{\mathrm{H}}\right)$ and $\alpha_{\mathrm{B}}$ the case-B hydrogen recombination coefficient. We note that the volume-averaged ionization parameter in expression (4) is a factor of 9/4 larger than the zero-age ionization parameter at the Strömgren radius used by Gutkin et al. (2016) and a factor of $3 / 4$ smaller than the quantity defined by equation (7) of Charlot \& Longhetti (2001). These different model-labelling choices are transparent to the CLOUDY calculations. Also, since $\langle U\rangle$ is proportional to $\left[Q(0) \epsilon^{2} n_{\mathrm{H}}\right]^{1 / 3}$, at fixed $\langle U\rangle$ and $n_{\mathrm{H}}$, there is a degeneracy in the calculations between the adopted normalisation of $Q(0)$ (via an effective mass of ionizing star cluster) and $\epsilon$.

The CLOUDY calculations to compute $T_{\lambda}^{\mathrm{HII}}\left(t^{\prime}\right)$ are performed in closed geometry, adopting a small inner radius of the gaseous nebula, $r_{\text {in }}=0.01 \mathrm{pc}$, to ensure spherical geometry. The photoionization calculations are stopped at the edge of the Hir region, when the electron density falls below 1 per cent of $n_{\mathrm{H}}$.

As noted by Vidal-García et al. (2017), the above standard CLOUDY calculations do not account for interstellar-line absorption in the ionized gas. In the following, we also wish to investigate the effects on nebular emission of interstellar-line absorption in the HII interiors and Hi envelopes of stellar birth clouds. To compute $T_{\lambda}^{\mathrm{HII}}\left(t^{\prime}\right) T_{\lambda}^{\mathrm{HI}}\left(t^{\prime}\right)$ in equation (3) in this case, we appeal to the prescription of Vidal-García et al. (2017, see their section 4), which extends the computations of Gutkin et al. (2016) to account for interstellar-line absorption in stellar birth clouds. This

1 These are based on the solar chemical abundances compiled by Bressan et al. (2012) from the work of Grevesse \& Sauval (1998), with updates from Caffau et al. (2011, see table 1 of Bressan et al. 2012), and small adjustments of the solar nitrogen $(-0.15 \mathrm{dex})$ and oxygen $(+0.10 \mathrm{dex})$ abundances relative to the mean values quoted in table 5 of Caffau et al. (2011, see Gutkin et al. 2016 for details). 
is achieved through the combination of CLOUDY with the general spectrum synthesis program SYNSPEC (e.g., Hubeny \& Lanz $2011)^{2}$ via an interactive program called CLOUDSPEC (Hubeny et al. 2000, see also Heap et al. 2001). For this purpose, the CLOUDY calculations are stopped when the kinetic temperature of the gas falls below $50 \mathrm{~K}$, assumed to define the Hi envelope of a typical stellar birth cloud (see Vidal-García et al. 2017 for more details).

We require a stellar population synthesis model to compute the spectral evolution of a single stellar generation, $S_{\lambda}\left[t^{\prime}, Z(t-\right.$ $\left.t^{\prime}\right)$ ], in equation (1). In most applications in this paper, we use the latest version of the Bruzual \& Charlot (2003) stellar population synthesis model (Charlot \& Bruzual, in preparation, hereafter $\mathrm{C} \& \mathrm{~B})$. This differs from the version used by Gutkin et al. (2016) in the inclusion of updated spectra of Wolf-Rayet (hereafter WR) stars from the Potsdam Wolf-Rayet (PoWR) model library (see Appendix A) and of main-sequence massive stars from Chen et al. (2015). When indicated, we also use the Binary Population and Spectral Synthesis (BPASS v2.2.1) models of Stanway \& Eldridge (2018) to explore the effects of binary interactions on the spectral evolution of young stellar populations, in particular the enhancement of extreme ultraviolet radiation by envelope stripping (of primary stars) and chemical homogeneisation (of rapidly rotating secondaries). We adopt throughout a Chabrier (2003) initial mass function (IMF), with lower mass cutoff $0.01 \mathrm{M}_{\odot}$ and upper mass cutoff in the range $100 \leq m_{\text {up }} \leq 600 \mathrm{M}_{\odot}$. IMF upper mass cutoffs well in excess of $100 \mathrm{M}_{\odot}$ have been suggested by models and observations of massive, low-metallicity star clusters (e.g., Crowther et al. 2016; Smith et al. 2016, see also Vink et al. 2011). For the star formation history, $\psi\left(t-t^{\prime}\right)$ in equation (1), we adopt either a delta function (Simple Stellar Population, hereafter SSP) or constant star formation rate. We consider ages of up to $50 \mathrm{Myr}$, as, even though 99.9 per cent of $\mathrm{H}$-ionizing photons are produced at ages less than $10 \mathrm{Myr}$ in single-star models (e.g., Charlot \& Fall 1993; Binette et al. 1994), binary interactions can extend the production over longer timescales (e.g. Stanway et al. 2016).

\subsection{Density-bounded models}

We are also interested in the influence of LyC-photon leakage on the nebular emission from young star-forming galaxies. This leakage is generally thought to occur in two main possible ways: through holes carved into the neutral ISM by extreme galactic outflows ('picket-fenced' model), which can be traced by the presence of residual flux in the cores of saturated interstellar low-ionization absorption lines (e.g., C II $\lambda \lambda 1036,1037$; Heckman et al. 2011; Alexandroff et al. 2015) and reduced nebular emission-line equivalent widths (Zackrisson et al. 2013), but with no effect on line ratios (Zackrisson et al. 2017); or through density-bounded (i.e. optically thin to LyC photons) His regions, which are expected to lead to weak low-ionization emission lines, a small velocity spread of the Ly $\alpha$ double-peaked emission and large ratios of high- to low-ionization lines (Giammanco et al. 2005; Pellegrini et al. 2012; Jaskot \& Oey 2013; Zackrisson et al. 2013; Nakajima \& Ouchi 2014; Nicholls et al. 2014; Stasińska

2 See http://nova.astro.umd.edu/Synspec49/synspec.html et al. 2015; Jaskot \& Ravindranath 2016; Alexandroff et al. 2015; Izotov et al. 2018b; D’Agostino et al. 2019). We note that, in addition to these two commonly cited scenarios, direct ionizing radiation from runaway massive stars could also contribute significantly to LyC leakage (Conroy \& Kratter 2012). We focus here on the modelling of density-bounded HII regions, which is the only LyC-leakage scenario affecting ratios of nebular emission lines and also seems to be favoured by current observations (see Section 3.2 below).

\subsubsection{Modelling approach}

In the framework of photoionization modelling described in Section 2.1, we can write the (effective) time-dependent fraction of LyC photons escaping from a density-bounded His region ionized by a single stellar generation as

$f_{\mathrm{esc}}\left(t^{\prime}\right)=\frac{Q^{\mathrm{out}}\left(t^{\prime}\right)}{Q\left(t^{\prime}\right)}$,

where $Q\left(t^{\prime}\right)$ is the rate of LyC photons produced by the stellar population at age $t^{\prime}$, and $Q^{\text {out }}\left(t^{\prime}\right)$ the rate emerging from the nebula at that age. The quantity $Q^{\text {out }}\left(t^{\prime}\right)$ encompasses both the fraction of LyC photon initially produced by stars that escape from the nebula, and the LyC photons created within the nebula (via free-bound emission) that also escape from it. This latter contribution is negligible, as the ionizing recombination continuum amounts to less than 0.001 per cent of $Q\left(t^{\prime}\right)$ for an ionization-bounded nebula.

It is convenient to parametrize density-bounded models in terms of the zero-age optical depth to LyC photons, rather than the H-column density of the Hir region. This is because at fixed $\mathrm{H}$-column density, the optical depth, which controls the quantity $f_{\text {esc }}$ we are interested in, can vary greatly depending on gas composition and ionization state. While CLOUDY computes the optical depth to LyC photons in a self-consistent way, it is useful, for the purpose of describing the sensitivity of observed line ratios on model parameters (Section 4), to express the optical depth at wavelength $\lambda$ and radius $r$ at age $t^{\prime}=0$ as the sum of the optical depths arising from the gas and dust phases,

$\tau_{\lambda}(r)=\tau_{\lambda, \text { gas }}(r)+\tau_{\lambda, \text { dust }}(r)$.

The optical depth from neutral hydrogen and other gaseous species is (e.g. Osterbrock \& Ferland 2006)

$\tau_{\lambda, \text { gas }}(r)=\sigma_{\lambda}\left(\mathrm{H}^{0}\right) N\left(\mathrm{H}^{0}, r\right)+\sum_{\mathrm{X}, i} \sigma_{\lambda}\left(\mathrm{X}^{+i}\right) N\left(\mathrm{X}^{+i}, r\right)$,

where $\sigma_{\lambda}\left(\mathrm{H}^{0}\right)$ and $\sigma_{\lambda}\left(\mathrm{X}^{+i}\right)$ are the monochromatic absorption cross-sections of neutral hydrogen and element $\mathrm{X}$ (with atomic number $\geq 2)$ in ionization state $+i$, and $N\left(\mathrm{H}^{0}, r\right)$ and $N\left(\mathrm{X}^{+i}, r\right)$ the column densities of $\mathrm{H}^{0}$ and $\mathrm{X}^{+i}$ out to radius $r$. Both $N\left(\mathrm{H}^{0}, r\right)$ and $N\left(\mathrm{X}^{+i}, r\right)$ are proportional to the gas filling factor $\epsilon$ (Section 2.1). The optical depth arising from dust can be expressed as

$\tau_{\lambda, \text { dust }}(r)=\sigma_{\lambda \text {,dust }} \xi_{\mathrm{d}} Z N_{\mathrm{H}}(r)$,

where $\sigma_{\lambda, \mathrm{d}}$ is the dust absorption cross-section at wavelength $\lambda$, and $N_{\mathrm{H}}(r)=\epsilon n_{\mathrm{H}} r$ the H-column density at radius $r$.

In practice, we parametrise density-bounded models in 

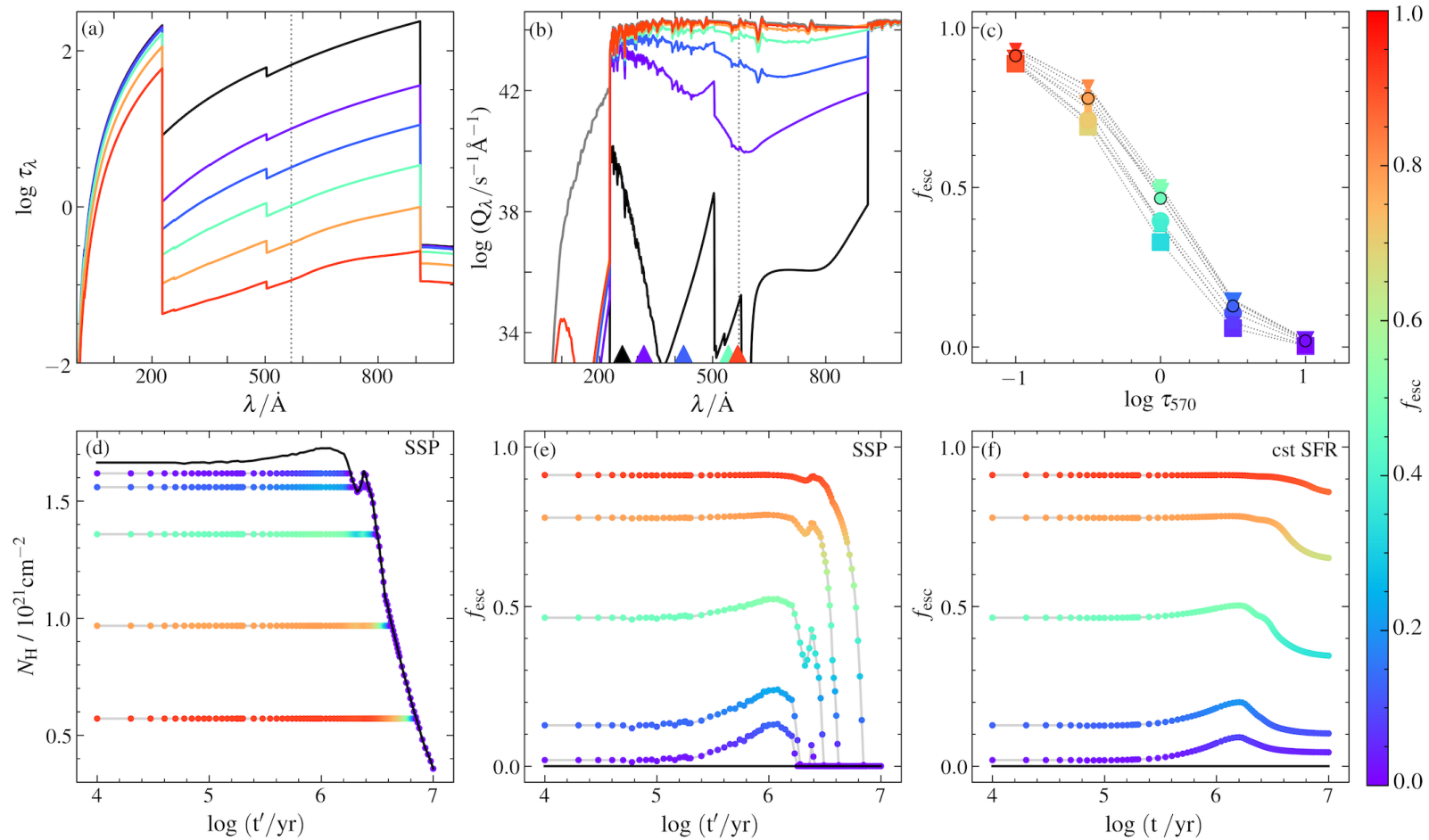

Figure 1. Relationship between zero-age optical depth to LyC photons, $\tau_{\lambda}$, fraction of escaping LyC photons, $f_{\text {esc }}$, and $\mathrm{H}$-column density, $N_{\mathrm{H}}$, in the density-bounded models of Section 2.2. (a) $\tau_{\lambda}$ plotted against $\lambda$ for models with $Z=0.002, \log \langle U\rangle=-2.0, \xi_{\mathrm{d}}=0.3, m_{\mathrm{up}}=300 \mathrm{M}_{\odot}$ and for 5 choices of $\tau_{570}$ (from -1.0 to +1.0 in steps of 0.5 , as identifiable from the dotted vertical line). Different colours reflect the $f_{\text {esc }}$ values of these models (see scale on the right), while the black line corresponds to the ionization-bounded model. (b) Monochromatic photon rate $Q_{\lambda}$ emerging at age zero from the models of panel (a), plotted against $\lambda$. Triangles indicate the photon-weighted mean wavelength of each spectrum. The grey curve shows the input stellar population spectrum, with photon-weighted mean wavelength marked by the dotted vertical line. (c) $f_{\text {esc }}$ plotted against $\tau_{570}$ for the same models as in (a) (black-contoured circles) and for models with different metallicities (upside-down triangles: $Z=0.0005$; squares: $Z=0.008$ ) and $\log \langle U\rangle=-3.0,-2.0$ and -1.0 (in order of increasing symbol size). (d) H-column density plotted against HII-region age for the same models as in (a). (e) fesc plotted against HII-region age for the same models as in (a). (f) Same as (e), but for a galaxy with constant star formation rate (i.e., adopting $\psi=$ cst in equation 1). In panels (d)-(f), different colours reflect the $f_{\text {esc }}$ values of the density-bounded models computed at discrete ages (see scale on the right),

terms of the zero-age optical depth of the Hit region to LyC photons with wavelength $\lambda=570 \AA$, noted $\tau_{570}$. This corresponds to the photon-rate-weighted mean wavelength of $\mathrm{H}$-ionizing radiation produced by a zero-age stellar population with metallicity $Z=0.002$ and IMF upper-mass cutoff $m_{\text {up }}=300 \mathrm{M}_{\odot}$ in the $\mathrm{C} \& \mathrm{~B}$ models. For chosen input parameters, including $\tau_{570}$, we run CLOUDY at age $t^{\prime}=0$ in the same way as described in the previous section for ionization-bounded HII regions, but stopping this time the calculation when the optical depth at $\lambda=570 \AA$ reaches $\tau_{570}$. At the end of the calculation, we record the $\mathrm{H}$ column density corresponding to this model of density-bounded nebula. Then, for all ages $t^{\prime}>0$, we compute the nebular emission with CLOUDY, stopping the calculation when the H-column density reaches that determined at $t^{\prime}=0$, or when the electron density falls below 1 per cent of $n_{\mathrm{H}}$.

\subsubsection{Properties of density-bounded models}

Fig. 1 illustrates the relationship between $\tau_{570}, f_{\text {esc }}$ and H-column density, $N_{\mathrm{H}}$, in these density-bounded models. Fig. 1a shows the wavelength dependence of the zero-age optical depth, $\tau_{\lambda}$ (equation 6), for models with fixed metallicity $Z=0.002$, ionization parameter $\log \langle U\rangle=-2.0$, dust-to-metal mass ratio $\xi_{\mathrm{d}}=0.3$, IMF upper mass cutoff $m_{\text {up }}=300 \mathrm{M}_{\odot}$, and for 5 choices of $\tau_{570}$, from -1.0 to +1.0 in steps of 0.5 . The curves are colour-coded to reflect the $f_{\text {esc }}$ values of these models. Also shown for comparison is the ionization-bounded model with same parameters (in black). The breaks in the curves correspond to the ionization potentials of helium (at 228 and $504 \AA$ ) and hydrogen (at $912 \AA$ ), which give rise to sharp features in the ionizing spectra emerging from these HII regions (Fig. 1b). Also, the increase in $\tau_{\lambda}$ at wavelengths from 228 to $912 \AA$ implies that the photon-weighted mean wavelength of ionizing photons emerging from the HıI region increases from high to low $\tau_{570}$ (as indicated by the triangles at the bottom of Fig. 1b). This also implies that ionizing photons with wavelengths less than $912 \AA$ can escape the nebula when the optical depth at the Lyman edge is unity.

In Fig. 1c, we show $f_{\text {esc }}$ as a function of $\tau_{570}$ for models with different metallicities, $Z=0.0005$ (upside-down triangles), 0.002 (circles) and 0.008 (squares), and different ionization parameters, $\log \langle U\rangle=-3.0,-2.0$ and -1.0 (in order of increasing 
symbol size). At fixed $\tau_{570}$, differences in $f_{\text {esc }}$ between these models arise from differences in the wavelength dependence of $\tau_{\lambda}$. Increasing $Z$ at fixed $\log \langle U\rangle$ implies a larger contribution to the optical depth by metals and dust, and hence, at fixed $\tau_{570}$, a smaller one by $\mathrm{H}^{0}$ (equations 6-8). This turns out to produce a flatter dependence of $\tau_{\lambda}$ on wavelength relative to that shown in Fig. 1a, which makes $f_{\text {esc }}$ drop at fixed $\tau_{570}$ in Fig. 1c. Also, increasing $\log \langle U\rangle$ at fixed $Z$ (which can be achieved by raising $\epsilon$ at fixed $Q$ in equation 4) makes $N_{\mathrm{H}}$, and hence, the optical depths from metals and dust, increase, implying a smaller $\mathrm{H}^{0} \mathrm{op}-$ tical depth at fixed $\tau_{570}$. This (and the higher ionization state of metals) again contributes to making $f_{\text {esc }}$ drop when $\log \langle U\rangle$ increases in Fig. 1c. The effect is largest around the critical regime $\tau_{570} \sim 1$, where $f_{\text {esc }}$ can change from 0.35 to 0.55 depending on the adopted metallicity and ionization parameter.

In Fig. 1d, we plot $N_{\mathrm{H}}$ as a function of HiI-region age for the same models with $Z=0.002$ and (zero-age) $\log \langle U\rangle=-2.0$ as in Fig. 1a. For the reference ionization-bounded model (black curve), $N_{\mathrm{H}}$ rises until ages around $1 \mathrm{Myr}$, as massive stars evolve on the main sequence, and then drops and exhibits a secondary peak around $2.5 \mathrm{Myr}$, when the hard ionizing radiation from hot WR stars induces a peak in $Q\left(t^{\prime}\right)$ (and , $\log \langle U\rangle$; equation 4). Then, at later ages, $N_{\mathrm{H}}$ drops as the supply of ionizing photons dries up. For density-bounded models, by design, $N_{\mathrm{H}}$ remains constant at all ages until $Q\left(t^{\prime}\right)$ drops enough for the region to become ionization-bounded, reducing $f_{\text {esc }}$ to zero (Fig. 1e). In the case of a galaxy with constant star formation rate (i.e., adopting $\psi=$ cst in equation 1$), f_{\text {esc }}$ does not reach zero at ages $t^{\prime} \gtrsim$ $10 \mathrm{Myr}$, as newly formed $\mathrm{H}$ I regions continue to maintain leakage of LyC photons (Fig. 1f).

It is also interesting to examine the dependence of the neutral-H column density, $N\left(\mathrm{H}^{0}\right)$, on $\tau_{570}$ and $N_{\mathrm{H}}$ in the densitybounded models of Fig. 1. Fig. 2a shows $N\left(\mathrm{H}^{0}\right)$ against $\tau_{570}$ for the same zero-age models with various metallicities and ionization parameters as in Fig. 1c. At fixed $\tau_{570}$, the drop in $N\left(\mathrm{H}^{0}\right)$ mentioned above to compensate the enhanced opacity from metals and dust when increasing $Z$ and $\langle U\rangle$ is clearly apparent in this diagram, especially at low $\tau_{570}$, when the outer $\mathrm{H}^{0}$ layer of the density-bounded $\mathrm{H}_{\mathrm{II}}$ regions is very thin [i.e., $N\left(\mathrm{H}^{0}\right) \lesssim 1.6 \times 10^{17} \mathrm{~cm}^{-2}$, the column density required to produce unit optical depth at the Lyman edge]. In Figs $2 \mathrm{~b}$ and $2 \mathrm{c}$, we plot $N\left(\mathrm{H}^{0}\right)$ against total $\mathrm{H}$-column density $N_{\mathrm{H}}$, for $Z=0.0005$ and 0.008 , respectively, and in each case for the same models as in Fig. 2a with $\log \langle U\rangle=-3.0,-2.0$ and -1.0 (the lines join models of fixed $\langle U\rangle$ ). As noted previously, $N_{\mathrm{H}}$ increases together with $\langle U\rangle$. Also, at fixed $\langle U\rangle$ and $\tau_{570}, N_{\mathrm{H}}$ is smaller for $Z=0.008$ than for $Z=0.0005$, because more ionizing photons are absorbed by metals and dust relative to hydrogen at higher $Z$. At fixed ionization parameter, decreasing $\tau_{570}$ relative to the ionization-bounded model firstly amounts to making $N\left(\mathrm{H}^{0}\right)$ decrease at nearly fixed $N_{\mathrm{H}}$, until the outer $\mathrm{H}^{0}$ layer of the $\mathrm{H}_{\text {II region }}$ is nearly peeled off [i.e., around $N\left(\mathrm{H}^{0}\right) \sim 1.6 \times 10^{17} \mathrm{~cm}^{-2}$ ]. Further reducing $\tau_{570}$ requires a drop in the optical depth to $\mathrm{LyC}$ photons arising from metals and dust, and hence smaller $N_{\mathrm{H}}$. The transition between the two regimes occurs at smaller $f_{\text {esc }}$ for $Z=0.008$ (Fig. 2c) than for $Z=0.0005$ (Fig. 2b), because of the larger metal and dust optical depths at higher $Z$.

\subsubsection{Implications for emission-line properties}

We now turn to the emission-line properties of these densitybounded models. Figs $3 \mathrm{a}$ and $3 \mathrm{~b}$ show the fractions of total $\mathrm{C}$ abundance in the form of $\mathrm{C}^{2+}$ (dashed lines) and $\mathrm{C}^{3+}$ (solid lines), as a function of radius, in three reference ionization-bounded models with $\log \langle U\rangle=-3.0,-2.0$ and -1.0 (in order of increasing line thickness), for $Z=0.0005$ and 0.008 , respectively, at age $t^{\prime}=0$. The cutoff radii of the density-bounded models with different $\tau_{570}$ and $\log \langle U\rangle$ from Fig. $2 \mathrm{~b}$ are indicated by triangles at the bottom of each panel. At fixed $\langle U\rangle$, the fractional abundance of $\mathrm{C}^{3+}$ is largest in the inner, highly-ionized parts of the nebula, while $\mathrm{C}^{2+}$ dominates on the outer, lower-ionization parts. Increasing $\langle U\rangle$ (which can be achieved by raising $\epsilon$ at fixed $Q$ in equation 4) increases the probability for carbon to be multiply ionized in the inner parts of the nebula, causing an inner $\mathrm{C}^{4+}$ zone (not shown) to develop, while the $\mathrm{C}^{3+}$ zone thickens to the detriment of the $\mathrm{C}^{2+}$ zone. At fixed $\tau_{570}$, the cutoff radii corresponding to density-bounded models with different $\langle U\rangle$ and $Z$ sample different global abundances of $\mathrm{C}^{2+}$ and $\mathrm{C}^{3+}$ in Figs $3 \mathrm{a}$ and $3 b$.

The implications for the C III] $\lambda 1908$ and C IV $\lambda 1549$ emission-line properties of models with different $f_{\text {esc }}$ are shown in the bottom panels of Fig. 3. Figs 3c and 3d show the equiv-

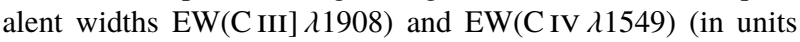
of the equivalent widths in the ionization-bounded case), respectively, as a function of $f_{\text {esc }}$, for the same zero-age models with different $\langle U\rangle$ and $Z$ as in Fig. 1c above. Fig. 3e shows the C III] $\lambda 1908 / C$ IV $\lambda 1549$ line-luminosity ratio. As expected from Figs $3 \mathrm{a}$ and $3 \mathrm{~b}$, the gradual removal of the outer low-ionization zone when $f_{\text {esc }}$ rises makes EW(C III] $\left.\lambda 1908\right)$ decrease more rapidly than $\mathrm{EW}(\mathrm{C}$ IV $\lambda 1549)$, and the C III] $\lambda 1908 / \mathrm{C}$ IV $\lambda 1549$ ratio drop, the strengths of these effects increasing with both $\langle U\rangle$ and $Z$. We note that, for low $\langle U\rangle$ and $Z$, the rise in $\mathrm{EW}(\mathrm{C}$ IV $\lambda 1549)$ when $f_{\text {esc }}$ increases in Fig. $3 \mathrm{c}$ is caused by the drop in recombination-continuum flux at nearly constant line luminosity, since the C IV $\lambda 1549$ zone (thin solid lines in Figs 3a and $3 b$ ) is unaffected by the cuts in $N_{\mathrm{H}}$ (small coloured triangles; see also Raiter et al. 2010; Jaskot \& Ravindranath 2016). In Fig. 4, we show the analogues of Figs $3 \mathrm{a}$ and $3 \mathrm{e}$ for the [O II] $\lambda 3727$ and [O III $] \lambda 5007$ lines. The fractional abundances of $\mathrm{O}^{2+}$ and $\mathrm{O}^{+}$ (Fig. 4a) exhibit a dependence on radius similar to that of $\mathrm{C}^{2+}$ and $\mathrm{C}^{3+}$ (Fig. 3a), except that the outer low-ionization $\mathrm{O}^{+}$zone is thinner than the outer $\mathrm{C}^{2+}$ zone at all ionization parameters. This causes the $[\mathrm{O} \mathrm{II}] \lambda 3727 /[\mathrm{O}$ III] $\lambda 5007$ ratio (Fig. 4b) to drop more steeply than the C III] $\lambda 1908 / \mathrm{C}$ IV $\lambda 1549$ ratio (Figs 3e) when $f_{\text {esc }}$ increases, until the $\mathrm{O}^{+}$zone disappears.

So far, we have described the emission-line properties of density-bounded HII-region models at age $t^{\prime}=0$ only. Fig. 5 shows the evolution of the C III] $\lambda 1908$ (Fig. 5a), C IV $\lambda 1549$ (Fig. 5b), [O II $] \lambda 3727$ (Fig. 5d) and [O III] $\lambda 5007$ (Fig. 5e) emission-line luminosities as a function of $t^{\prime}$ for the same models with $Z=0.002, \log \langle U\rangle=-2.0, \xi_{\mathrm{d}}=0.3, m_{\text {up }}=300 \mathrm{M}_{\odot}$ and 5 choices of $\tau_{570}$ as in Fig. 1d. As in the case of $N_{\mathrm{H}}$ in Fig. 1d, the luminosity of emission lines in the reference ionization-bounded model (black curve in Figs $5 \mathrm{a}-5 \mathrm{~b}$ and $5 \mathrm{~d}-5 \mathrm{e}$ ) reaches a maximum at ages around $1 \mathrm{Myr}$ and exhibits a secondary peak when the hard ionizing radiation from hot WR stars kicks in, around 2.5 Myr. Then, after the most massive stars have died, line emis- 

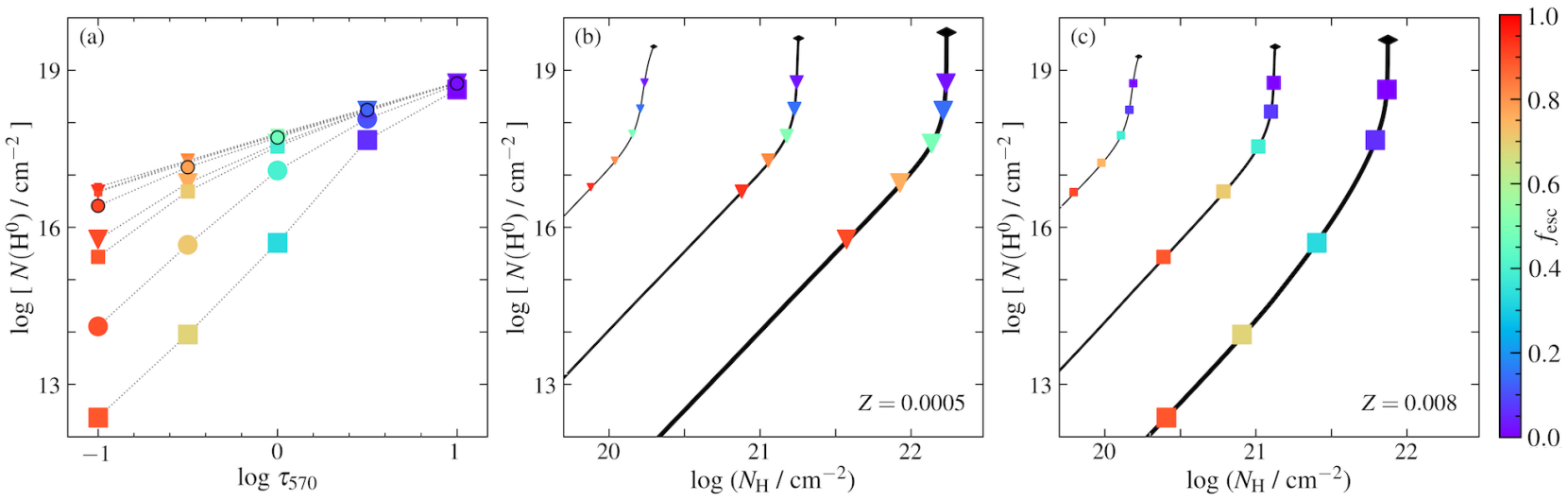

Figure 2. Relationship between optical depth to LyC photons at $\lambda=570 \AA$, $\tau_{570}$, neutral-H column density, $N\left(\mathrm{H}^{0}\right)$, and total $\mathrm{H}$-column density, $N_{\mathrm{H}}$, at age $t^{\prime}=0$ in the density-bounded models of Section 2.2. (a) $N\left(\mathrm{H}^{0}\right)$ plotted against $\tau_{570}$ for the same models as in Fig. 1c. (b) $N\left(\mathrm{H}^{0}\right)$ plotted against $N_{\mathrm{H}}$ for the subset of models in (a) with metallicity $Z=0.0005$. Lines join models of fixed $\log \langle U\rangle=-3.0,-2.0$ and -1.0 (in order of increasing thickness). At the top of each line, a black diamond indicates the location of the ionization-bounded model. (c) Same as (b), but for $Z=0.008$.
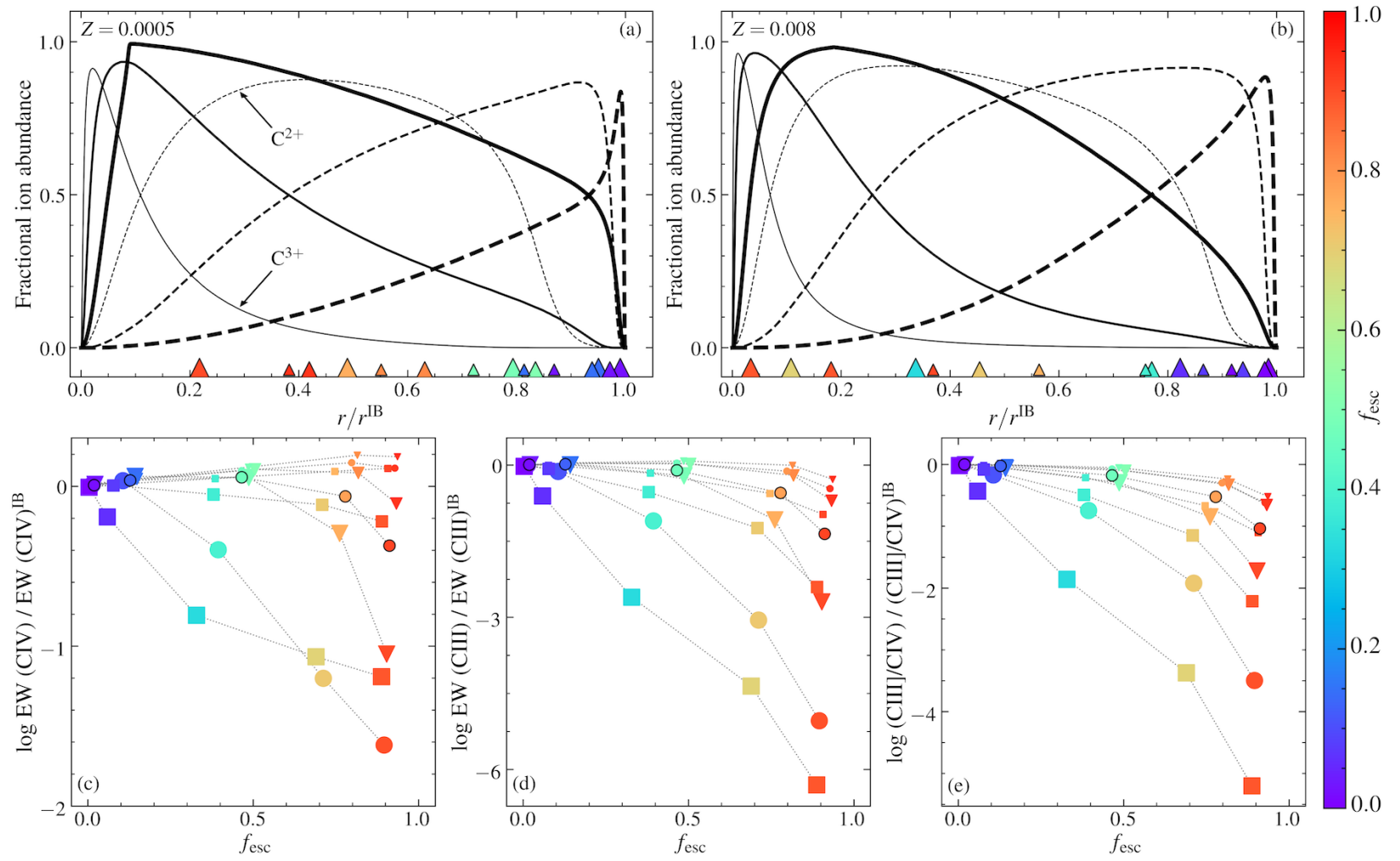

Figure 3. Carbon emission-line properties at age $t^{\prime}=0$ in the density-bounded models of Section 2.2. (a) Fractional abundances of $\mathrm{C}^{2+}$ (dashed lines) and $\mathrm{C}^{3+}$ (solid lines) plotted against radius $r$ (in units of the ionization-bounded HII-region radius, $r^{\mathrm{IB}}$ ), for reference ionization-bounded models with $Z=0.0005, \xi_{\mathrm{d}}=0.3, m_{\mathrm{up}}=300 \mathrm{M}_{\odot}$ and 3 values of the ionization parameter, $\log \langle U\rangle=-3.0,-2.0$ and -1.0 (in order of increasing line thickness). Triangles at the bottom locate the cutoff radii of the models with different $\tau_{570}$ and $\log \langle U\rangle$ (in order of increasing symbol size) of Fig. 2b. (b) Same as (a), but for $Z=0.008$. (c) Equivalent width of the C IV $\lambda 1549$ nebular emission line (in units of the equivalent width in the ionization-bounded case) plotted against $f_{\text {esc }}$, for the same density-bounded models as in Fig. 1c. (d) Same as (c), but for the equivalent width of C III] $\lambda 1908$. (e) Same as (c), but for the C III] $\lambda 1908 / C$ IV $\lambda 1549$ emission-line luminosity ratio.

sion fades. The secondary peak is most prominent in the evolution of the CIV $\lambda 1549$ luminosity, since C IV requires the most energetic photons to be produced $\left(E_{\text {ion }}>47.9 \mathrm{eV}\right.$, compared to $35.1 \mathrm{eV}$ for $\mathrm{O}$ III). In contrast, the [O II] $\lambda 3727$ luminosity, which requires the least energetic photons $\left(E_{\mathrm{ion}}>13.6 \mathrm{eV}\right.$ to produce O II, compared to $24.4 \mathrm{eV}$ for $\mathrm{C}$ III) does not drop as sharply as that of the other three lines at ages greater than a few Myr. For density-bounded models, the gradual removal of the outer low-ionization envelope when $\tau_{570}$ decreases reduces the $[\mathrm{O}$ II $] \lambda 3727$ luminosity more strongly than the C III] $\lambda 1908$, 

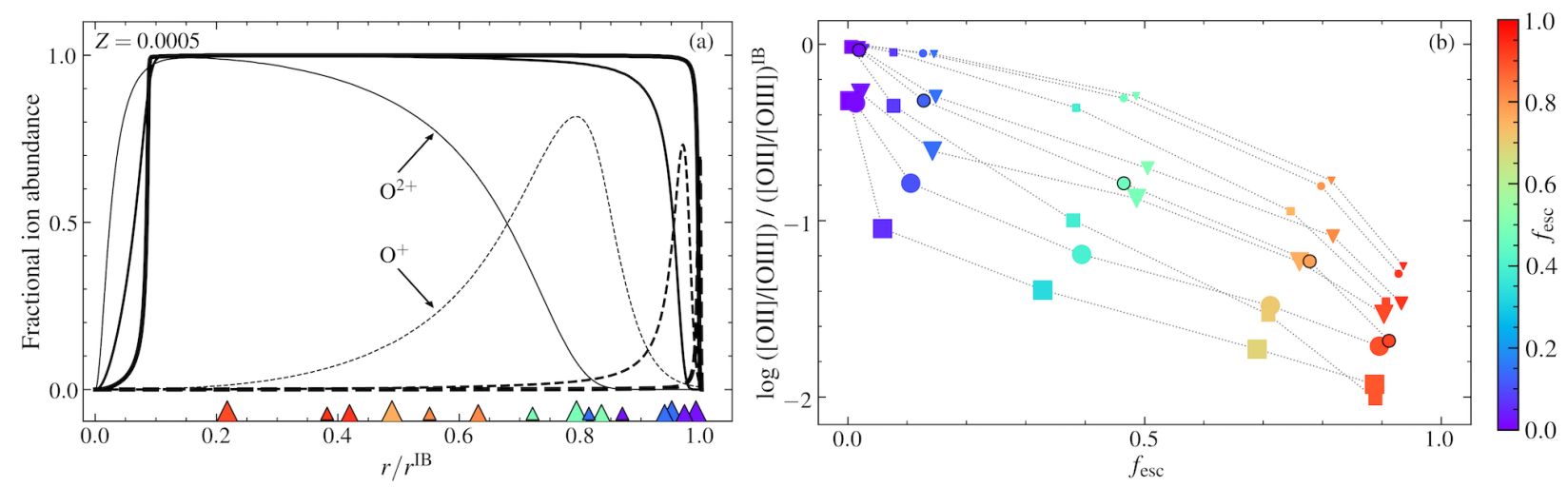

Figure 4. Oxygen emission-line properties at age $t^{\prime}=0$ in the density-bounded models of Section 2.2. (a) Same as Fig. 3a, but for the fractions of $\mathrm{O}^{+}$ (dashed lines) and $\mathrm{O}^{2+}$ (solid lines). (b) Same as Fig. 3e, but for the [O II] $] 33727 /[\mathrm{O}$ III $] \lambda 5007$ emission-line luminosity ratio.
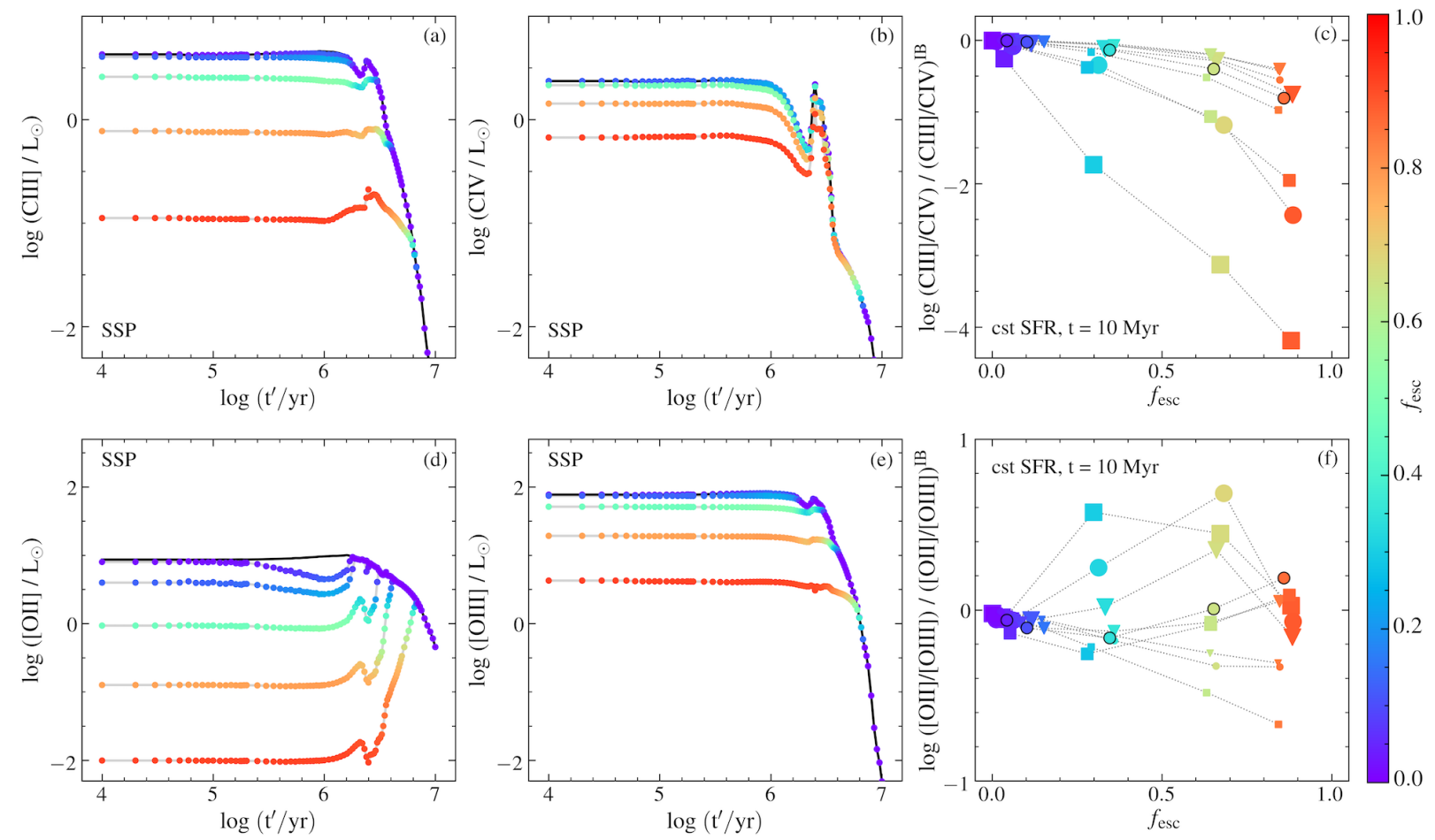

Figure 5. Emission-line properties of the density-bounded models of Section 2.2. (a) C III] $\lambda 1908$ emission-line luminosity plotted against HII-region age $t^{\prime}$ for the same models with $Z=0.002, \log \langle U\rangle=-2.0, \xi_{\mathrm{d}}=0.3, m_{\mathrm{up}}=300 \mathrm{M}_{\odot}$ and 5 choices of $\tau_{570}$ as in Fig. $1 \mathrm{~d}$. (b) Same as (a), but for C IV $\lambda 1549$. (c) C III] $\lambda 1908 / \mathrm{C}$ IV $\lambda 1549$ emission-line luminosity ratio for the same models with different $Z$ and $\log \langle U\rangle$ as in Fig. 3e, but at age $t=10 \mathrm{Myr}$ for a galaxy with constant star formation rate (i.e., adopting $\psi=$ cst in equation 1). (d) Same as (a), but for [O II] $\lambda 3727$. (e) Same as (a), but

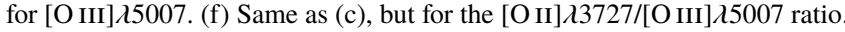

O III] $\lambda 1664$ and CIV $\lambda 1549$ ones at early ages, until the ionizing flux has dropped low enough for the nebula to become ionization-bounded. The low-ionization zone reappears, causing a sharp rise in $[\mathrm{O}$ II $] \lambda 3727$ luminosity at ages $t^{\prime} \gtrsim 3 \mathrm{Myr}$.

In Figs $5 \mathrm{c}$ and $5 f$, we show the resulting dependence on $f_{\text {esc }}$ of the C III] $\lambda 1908 / \mathrm{C}_{\text {IV }} \lambda 1549$ and [O II $] \lambda 3727 /[\mathrm{O}$ III $] \lambda 5007$ emission-line luminosity ratios, respectively, at age $t=10 \mathrm{Myr}$ for a galaxy with constant star formation rate (i.e., adopting $\psi=$ cst in equation 1), for the same models with different $Z$ and $\log \langle U\rangle$ as in Fig. 3e. The results for C III] $\lambda 1908 / C$ IV $\lambda 1549$ are very similar to those described above for single zero-age HII regions (Fig. 3e), as expected from the similar effect of reducing $\tau_{570}$ on the evolution of the C III] $\lambda 1908$ and C IV $\lambda 1549$ luminosities (Figs 5a and 5b). Instead, the dependence of $[\mathrm{O}$ II $] \lambda 3727 /[\mathrm{O} \mathrm{III}] \lambda 5007$ on $f_{\text {esc }}$ in Fig. $5 \mathrm{f}$ differs from that 
found for zero-age Hil regions in Fig. 4b (for $\log \langle U\rangle \geq-2.0$ ). For $\tau_{570}=1$, for example, corresponding roughly to $f_{\mathrm{esc}} \approx 0.3$ in the different models of Fig. 5f (and $f_{\text {esc }} \approx 0.5$ at $t^{\prime}=0$ in the single HII-region model of Fig. 5d), the rise in [O II] $\lambda 33727$ luminosity (Fig. 5d) and corresponding drop in [O III] $\lambda 5007$ luminosity (Fig. 5e) at ages $t^{\prime} \gtrsim 3 \mathrm{Myr}$ in the evolution of single HII regions can cause [O II $] \lambda 3727 /[\mathrm{O}$ III $] \lambda 5007$ to exceed the ionization-bounded value at $t=10 \mathrm{Myr}$ for a galaxy with constant star formation rate, especially for large $\langle U\rangle$ (Fig. 5f).

Hence, while a small [O II] $] 33727 /[\mathrm{O}$ III $] \lambda 5007$ ratio can be a clue of significant LyC leakage in models of individual density-bounded Hin regions, this is not the case for model galaxies containing several generations of Hir regions (see also Jaskot \& Oey 2013). It is worth noting that the models presented here are highly idealized, and that, in practice, a galaxy will contain different types of Hiı regions with different optical depths to LyC photons, metallicities and ionization parameters. In any case, the complex dependence of the C III] $\lambda 1908 / C$ IV $\lambda 1549$ and [O II] $] \lambda 3727 /[\mathrm{O}$ III $] \lambda 5007$ ratios on $f_{\text {esc }}$ identified in Figs 3-5 above illustrates the difficulty of observationally tracking galaxies which lose significant amounts of LyC photons. We will return to this point in Section 5.

\subsection{Nebular emission from AGN}

To explore the influence of an AGN on the nebular emission from a young star-forming galaxy, we use photoionization calculations of AGN narrow-line regions based on the approach of Feltre et al. (2016). This relies on a parametrization of CLOUDY similar to that described above in terms of hydrogen density $n_{\mathrm{H}}$, gas metallicity $Z, \mathrm{C} / \mathrm{O}$ ratio, dust-to-metal mass ratio $\xi_{\mathrm{d}}$ and volumeaveraged ionization parameter $\langle U\rangle$, but using the emission from an accretion disc in place of equation (1) as input radiation. The spectral energy distribution of the accretion disc is parametrized as

$S_{\nu} \propto\left\{\begin{array}{lll}v^{\alpha} & \text { at wavelengths } & 0.001 \leq \lambda / \mu \mathrm{m} \leq 0.25, \\ v^{-0.5} & \text { at wavelengths } & 0.25<\lambda / \mu \mathrm{m} \leq 10.0, \\ v^{2} & \text { at wavelengths } & \lambda / \mu \mathrm{m}>10.0 .\end{array}\right.$

We adopt here for simplicity a fixed slope $\alpha=-1.7$ at high energies (Zheng et al. 1997; Lusso et al. 2015) and a fixed gas density $n_{\mathrm{H}}=10^{3} \mathrm{~cm}^{-3}$ in the narrow-line region (e.g., Osterbrock \& Ferland 2006). The CLOUDY calculations are performed in 'open geometry' (see Feltre et al. 2016, for more details).

The models adopted here differ from those originally published by Feltre et al. (2016) in that they are computed using version c17.00 of CLOUDY (Ferland et al. 2017) and include dissipative microturbulence in the gas clouds in the narrow-line region (with a microturbulence velocity of $100 \mathrm{~km} \mathrm{~s}^{-1}$ ) and a smaller inner radius of this region $(90 \mathrm{pc}$ instead of $300 \mathrm{pc}$, for an AGN luminosity of $10^{45} \mathrm{erg} \mathrm{s}^{-1}$ ). These parameters were found to better reproduce the observed ultraviolet emission-line spectra (in particular, the $\mathrm{N} v \lambda 1240$ emission) of a sample of 90 type-2 AGN at redshifts $z=1.5-3.0$ (Mignoli et al. 2019).

\subsection{Nebular emission from radiative shocks}

We are also interested in the effects of a contribution by shockionized gas to the nebular emission from actively star-forming galaxies. We appeal to the $3 \mathrm{MdBs}$ database ${ }^{3}$ of fully radiative shock models recently computed by Alarie \& Morisset (2019) using the MAPPINGS V shock and photoionization code (Sutherland \& Dopita 2017). The models (computed in plane-parallel geometry) are available for the same sets of element abundances as adopted in the stellar and AGN photoionization models described in Sections 2.1-2.3 above (albeit for only two values of the $\mathrm{C} / \mathrm{O}$ ratio: 0.11 and 0.44 ). Metal depletion on to dust grains is not included in this case, as in fast shocks, dust can be efficiently destroyed by grain-grain collisions, through both shattering and spallation, and by thermal sputtering (Allen et al. 2008). The other main adjustable parameters defining the model grid are the shock velocity (from $10^{2}$ to $10^{3} \mathrm{~km} \mathrm{~s}^{-1}$ ), pre-shock density (from 1 to $10^{4} \mathrm{~cm}^{-2}$ ) and transverse magnetic field (from $10^{-4}$ to $10 \mu \mathrm{G})$. The pre-shock density $\left(n_{\mathrm{H}}\right)$ and transverse magnetic field (noted $B$ ) have a much weaker influence than shock velocity on most emission lines of interest to us (see Section 4). Thus, in the following, to probe global trends in the influence of radiative shocks on the nebular emission from star-forming galaxies, we consider for simplicity models with fixed $n_{\mathrm{H}}=10^{2} \mathrm{~cm}^{-3}$ and $B=1 \mu \mathrm{G}$ in the full available range of shock velocities. We focus here on the predictions of models including nebular emission from both shocked and shock-precursor (i.e., pre-shock gas photoionized by the shock) gas (see Alarie \& Morisset 2019 for more details).

\section{OBSERVATIONAL CONSTRAINTS}

In this section, we build a reference sample of the nebular emission from metal-poor star-forming galaxies and LyC leakers at various redshifts, including also other star-forming galaxies and AGN, which we will use in Sections 4 and 5 to explore potentially discriminating signatures of the different adjustable parameters of the versatile models presented in Section 2. To this end, we wish to assemble a large homogeneous sample of observations of metal-poor star-forming galaxies at ultraviolet and optical wavelengths, by gathering from the literature data often analysed in independent ways using different models and assumptions. In the following, we assemble observations of such galaxies (Section 3.1), as well as of confirmed and candidate LyC leakers (Section 3.2) and other star-forming galaxies and AGN (Section 3.3) in a wide redshift range. We consider here only observational studies which gathered enough nebular emission-line properties to be plotted in at least one of the diagrams we investigate. Observations involving ultraviolet lines are presented in Fig.6, and those involving optical lines in Fig. 7. We comment on the general properties of this reference sample in Section 3.4.

${ }^{3}$ See http://3mdb.astro.unam.mx:3686 


\begin{tabular}{|c|c|c|c|c|c|c|}
\hline Reference & $z$ & Sample & $12+\log (\mathrm{O} / \mathrm{H})_{\mathrm{gas}}$ & Modelling & Physical parameters $^{a}$ & Comment \\
\hline Senchyna et al. $(2017,2019)$ & $<0.05$ & $\begin{array}{l}10 \text { extreme SF regions and } \\
6 \text { extremely metal-poor SF } \\
\text { galaxies from SDSS }\end{array}$ & $7.5-8.5$ & $\begin{array}{l}\text { BEAGLE } \\
\text { CLOUDY } 13.03 \\
\text { (dust physics) }\end{array}$ & $\begin{array}{c}-1.4<\log \mathrm{C} / \mathrm{O}<-0.7^{b} \\
-3.6<\log \langle U\rangle<-1.9^{b} \\
\text { sSFR } \sim 2-300 \mathrm{Gyr}^{-1}\end{array}$ & $\begin{array}{l}\text { Transition from primarily stellar to purely nebular He II } \lambda 1640 \\
\text { near } 12+\log (\mathrm{O} / \mathrm{H}) \lesssim 8.0 \text {; no evidence for shocks nor XRBs; }{ }^{b} \\
\text { strong C IV } \lambda 1549 \text { traces young ages, extremely low } Z \text { and } \alpha / \mathrm{Fe}\end{array}$ \\
\hline Berg et al. $(2016,2019)$ & $<0.14$ & $\begin{array}{l}32 \text { compact SF regions with } \\
\mathrm{EW}([\mathrm{O} \mathrm{III}] \lambda 5007)>50 \AA\end{array}$ & $7.4-8.0$ & $\begin{array}{l}\text { BPASS v2.14c } \\
\text { CLOUDY } 17.00 \\
\text { (no dust) }\end{array}$ & $\begin{array}{c}-1.0<\log \mathrm{C} / \mathrm{O}<-0.3 \\
-2.8<\log U_{0}<-1.8 \\
\text { SSFR } \sim 1-40 \mathrm{Gyr}^{-1}\end{array}$ & $\begin{array}{l}\text { No obvious AGN (C IV } \lambda 1549 / \mathrm{C} \text { III] } \lambda 1908<1) \text { nor shock } \\
([\mathrm{O} \text { I }] \lambda 6300 /[\mathrm{O} \text { III }] \lambda 5007<0.01 \text {; see Fig. } 15) \text { contribution }\end{array}$ \\
\hline Berg et al. (2018) & 1.8 & $\begin{array}{l}\text { Lensed, extreme-SF galaxy } \\
\text { SL2SJ021737-051329 }\end{array}$ & $\sim 7.5$ & $\begin{array}{l}\text { BPASS v2.14 } \\
\text { CLOUDY } 17.00 \\
\text { (no dust) }\end{array}$ & $\begin{array}{c}\log \mathrm{C} / \mathrm{O} \sim-0.8 \\
\log U_{0} \sim-1.5 \\
\text { sSFR } \sim 100 \mathrm{Gyr}^{-1}\end{array}$ & $\begin{array}{l}\text { Strong nebular He II } \lambda 1640 \text { not reproducible by models; no } \\
\text { obvious AGN nor shock contribution (Groves et al. 2004-AGN } \\
\text { and Allen et al. 2008-shock prescriptions deteriorate fits) }\end{array}$ \\
\hline Stark et al. (2014) & $1.5-3.0$ & 17 lensed, dwarf SF galaxies & $7.3-7.8$ & $\begin{array}{l}\text { BEAGLE-like } \\
\text { CLOUDY } 13.03 \\
\text { (dust physics) }\end{array}$ & $\begin{aligned} &-1.0<\log \mathrm{C} / \mathrm{O}<-0.3 \\
&-1.8<\log \langle U\rangle<-1.5^{d} \\
& \text { SSFR } \sim 2-40 \mathrm{Gyr}^{-1}\end{aligned}$ & $\begin{array}{l}\text { C III] } \lambda 1908 \text { detected in } 16 \text { systems; strongest }(E W>10 \AA) \\
\text { emitters show C IV } \lambda 1549 \text { emission, while nebular He II } \lambda 1640 \\
\text { is weak or non-detected in many systems }\end{array}$ \\
\hline Erb et al. (2010) & 2.3 & $\begin{array}{l}\text { Low-mass SF galaxy } \\
\text { Q2343-BX418 }\end{array}$ & $\sim 7.8$ & $\begin{array}{l}\text { STARBURST } 99^{e} \\
\text { CLOUDY } 08.00 \\
\text { (dust physics) }\end{array}$ & $\begin{array}{c}\log \mathrm{C} / \mathrm{O} \sim-0.6 \\
\log U_{0} \sim-1.0 \\
\mathrm{sSFR} \sim 16 \mathrm{Gyr}^{-1}\end{array}$ & Unlikely AGN contribution (C IV $\lambda 1549 / \mathrm{C}$ III] $\lambda 1908 \sim 0.3$ ) \\
\hline Amorín et al. (2017) & $2.4-3.5$ & $\begin{array}{l}10 \text { VUDS SF galaxies with } \\
\text { Ly } \alpha, \text { O III] } \lambda 1664 \text { and } \\
\text { C III] } \lambda 1908 \text { emission }\end{array}$ & $7.4-7.7$ & $\begin{array}{l}\text { POPSTAR }^{f} \\
\text { CLOUDY } 13.03 \\
\text { (dust physics) }\end{array}$ & $\begin{array}{c}-1.0<\log \mathrm{C} / \mathrm{O}<-0.4 \\
-2.3<\log U_{0}<-1.7 \\
\text { sSFR } \sim 5-50 \mathrm{Gyr}^{-1}\end{array}$ & $\begin{array}{l}\text { No obvious AGN contributions (from C IV } \lambda 1549, \text { He II } \lambda 1640 \text {, } \\
\text { C III] } \lambda 1908, \text { O III] } \lambda 1664 \text {; no X-ray emission nor broad lines) }\end{array}$ \\
\hline Nakajima et al. (2018) & $2-4$ & $\begin{array}{l}450 \text { VUDS SF galaxies with } \\
C \text { III }] \lambda 1908 \text { emission }(\mathrm{C})^{g} \\
43 \text { with } E W=10-20 \AA(B)^{g} \\
16 \text { with } E W>20 \AA(A)^{g}\end{array}$ & $\begin{array}{l}\sim 8.3(\mathrm{C}) \\
\sim 7.6(\mathrm{~B}) \\
\sim 7.8(\mathrm{~A})\end{array}$ & $\begin{array}{l}\text { POPSTAR } \\
\text { BPASS v2.0 } \\
\text { CLOUDY } 13.03 \\
\text { (dust physics) }\end{array}$ & $\begin{array}{c}\log \mathrm{C} / \mathrm{O} \sim-0.5(\mathrm{C}), \\
-0.3(\mathrm{~B}) \text { and }-0.4(\mathrm{~A}) \\
\log U_{0} \sim-2.9(\mathrm{C}), \\
-1.7(\mathrm{~B}) \text { and }-1.6(\mathrm{~A})\end{array}$ & $\begin{array}{l}\text { AGN contribution to ionizing radiation required in strongest } \\
\text { C III] } \lambda 1908 \text { emitters, irrespective of the inclusion of binary } \\
\text { stars in the stellar population modelling }\end{array}$ \\
\hline Nanayakkara et al. (2019) & $2-4$ & $\begin{array}{l}12 \text { MUSE SF galaxies with } \\
\text { He II } \lambda 1640 \text { emission }\end{array}$ & $7.9-8.6$ & $\begin{array}{l}\text { BPASS v2.1 } \\
\text { CLOUDY } 13.03 \\
\text { (no dust) }\end{array}$ & $\begin{array}{c}\log \mathrm{C} / \mathrm{O} \sim-0.4 \\
-2.5<\log U_{0}<-1.5\end{array}$ & $\begin{array}{l}\text { Rest-frame He II } \lambda 1640 \text { equivalent widths of } 0.2-10 \AA \AA \text { not } \\
\text { reproducible by models with or without binary stars }\end{array}$ \\
\hline Vanzella et al. (2017) & 3.2 & $\begin{array}{l}\text { Lensed double-super star } \\
\text { cluster ID14 }\end{array}$ & $\sim 7.7$ & & sSFR $\sim 20 \mathrm{Gyr}^{-1}$ & $\begin{array}{l}\text { Emission-line spectrum from C IV } \lambda 1549 \text { through } \\
C_{\text {III }} \lambda 1908 \text { consistent with photoionization by stars }\end{array}$ \\
\hline Fosbury et al. (2003) & 3.4 & $\begin{array}{l}\text { Lensed HII galaxy } \\
\text { RX J0848+4456 }\end{array}$ & $\sim 7.6$ & $\begin{array}{l}\text { Pure blackbody } \\
\text { MAPPINGS V Ic } \\
\text { (no dust) }\end{array}$ & $\log U_{0} \sim-1.0$ & $\begin{array}{l}\text { Absence of } \mathrm{NV} \lambda 1240 \text { and weakness of } \mathrm{N} \text { III] } \lambda 1750 \\
\text { taken as evidence against photoionization by an AGN }\end{array}$ \\
\hline Schmidt et al. (2017) & 6.1 & Lensed Ly $\alpha$ galaxy & $<8.3$ & & $\mathrm{sSFR} \sim 40 \mathrm{Gyr}^{-1}$ & $\begin{array}{l}\text { Emission-line spectrum from C IV } \lambda 1549 \text { through } \\
\text { C III] } \lambda 1908 \text { consistent with photoionization by stars }\end{array}$ \\
\hline Stark et al. (2015) & 7.0 & Lensed Ly $\alpha$ galaxy & $\sim 7.0$ & $\begin{array}{l}\text { BEAGLE-like } \\
\text { CLOUDY } 13.03 \\
\text { (dust physics) }\end{array}$ & $\log \langle U\rangle \sim 1.0$ & $\begin{array}{l}\text { C IV } \lambda 1549 \text { emission and upper limits on He II } \lambda 1640 \\
\text { and O III] } \lambda 1664 \text { emission consistent with photoionization } \\
\text { by both an AGN and stars }\end{array}$ \\
\hline Laporte et al. (2017) & 7.1 & $\begin{array}{l}{[\mathrm{O} \text { III] }] \text { ג5007-strong SF }} \\
\text { galaxy COSY }\end{array}$ & & & & $\begin{array}{l}\text { Prominent NV } \lambda 1240 \text { (and He II } \lambda 1640 \text { ) emission supports } \\
\text { photoionization by an AGN }\end{array}$ \\
\hline
\end{tabular}

${ }^{a} U_{0}$ refers to the ionization parameter at the inner edge of the gas cloud, which, in models with spherical geometry, corresponds to the inner radius of the HII region and is roughly 3 times larger than the volume-averaged ionization parameter $\langle U\rangle$ described in Section 2.1. ${ }^{b}$ Pertains to the 10 extreme SF regions studied by Senchyna et al. (2017). ${ }^{c}$ Berg et al. (2016) used STARBURST99. ${ }^{d}$ Range spanned by the 4 most extreme C III] $\lambda 1908$-emitting galaxies in the sample. ${ }^{e}$ (Mollá et al. 2009). ${ }^{f}$ (Leitherer et al. 1999, 2014). ${ }^{g}$ Letter referring to te corresponding sample in Nakajima et al. (2018) 


\subsection{Metal-poor star-forming galaxies}

We list in Table 1 the main characteristics of 13 samples of low-metallicity, actively star-forming galaxies. The samples are arranged in order of increasing redshift. In each case, we indicate the nature of the sample; the published gas-phase oxygen abundances of galaxies; the modelling tools used to interpret the observations (ionizing stellar population spectra and photoionization model); the constraints derived on physical parameters such as $\mathrm{C} / \mathrm{O}$ ratio, ionization parameter and specific star formation rate; and the main conclusions drawn in the original studies. The gas-phase oxygen abundance, $12+\log (\mathrm{O} / \mathrm{H})_{\text {gas }}$, is usually estimated using the direct- $T_{\mathrm{e}}$ method (see section 5.1 of Gutkin et al. 2016 for potential caveats of this method), and otherwise through photoionization calculations, including or not (in which case the total gas+dust-phase $\mathrm{O}$ abundance is not computed) depletion of oxygen onto dust grains. Also, we note that the differences in model analyses between the different studies in Table 1 go beyond the listed details. For example, the ionizing stellar population spectra can refer to different IMF shapes and upper-mass limits, star formation histories and ages. We do not focus on such differences here, as our main goal is to provide rough estimates of the characteristics of the various sample, which we will compare globally with a homogeneous set of models in Section 4. We now briefly describe these samples.

In the nearby Universe $(z \lesssim 0.1), H S T / C O S$ observations have brought valuable insight into the rest-ultraviolet properties of metal-poor star-forming galaxies with hard ionizing spectra. Senchyna et al. (2017) observed 10 galaxies from the sample of He II $\lambda 4686$-emitting, Sloan Digital Sky Survey (SDSS) starforming galaxies of Shirazi \& Brinchmann (2012), Senchyna et al. (2019) 6 extremely metal-poor $\left(Z / Z_{\odot} \lesssim 0.1\right)$ galaxies from the SDSS sample of Morales-Luis et al. (2011) and Berg et al. (2016, 2019) 32 compact, ultraviolet-bright, SDSS starforming galaxies with [O III] $\lambda 5007$ emission equivalent widths larger than $50 \AA$. Objects in these samples show no sign of AGN activity and range from high-ionization HII regions embedded in larger galaxies to blue compact dwarf galaxies. They can reach C III] $\lambda 1908$ equivalent widths as large as $\sim 15 \AA$, similar to those found in galaxies at redshifts $z>6$ (see below). Senchyna et al. (2017) identify a marked transition with decreasing metallicity around $12+\log (\mathrm{O} / \mathrm{H}) \approx 8.0$, from stellar-wind dominated to nebular-dominated He II $\lambda 1640$ and C IV $\lambda 1549$ emission. Analysis with the BEAGLE code (Chevallard \& Charlot 2016), allows them to reproduce all the stellar (e.g., C IV $\lambda 1549$ P-Cygni and broad-He II $\lambda 1640$ wind features) and nebular ultraviolet/optical emission-line properties of their sample (see also Chevallard et al. 2018), except for the strong nebular He II $\lambda 1640$ emission in the most metal-poor systems, which does not seem to be reproducible by any current stellar population synthesis prescription. Like Berg et al. $(2016,2019)$, they do not find any strong evidence for a contribution by radiative shocks to the nebular emission of galaxies in their sample.

At intermediate redshifts ( $2 \lesssim z \lesssim 4)$, spectroscopic observations with large optical telescopes have allowed detailed studies of the rest-ultraviolet spectra of unusually bright or lensed, dwarf star-forming galaxies (Table 1). The galaxies in these sample exhibit spectral characteristics typical of high-ionization, metalpoor star-forming galaxies. Remarkably, they also often show strong nebular He II $\lambda 1640$ emission, which cannot be reproduced by any current stellar population synthesis models, even when including enhanced production of hard ionizing radiation via binary mass transfer (Berg et al. 2018; Nakajima et al. 2018; Nanayakkara et al. 2019). Significant contribution from a luminous AGN is disfavoured in most cases, based on the weakness of $\mathrm{N} v \lambda 1240$, the low observed C IV $\lambda 1549 / \mathrm{C}$ III] $\lambda 1908$ ratio and the lack of X-ray detection and broad emission lines, standard optical (e.g. Baldwin et al. 1981, hereafter BPT) diagnostic diagrams being generally not available in this redshift range (Stark et al. 2014; Erb et al. 2010; Amorín et al. 2017; Vanzella et al. 2017; Berg et al. 2018; Nanayakkara et al. 2019). This is not the case for Nakajima et al. (2018, we adopt here the 3 composite spectra representative of classes A, B and C from this sample, with additional data from Le Fèvre et al. 2019 for classes A and B; see Table 1), who argue that an AGN contribution is required to account for the He II $\lambda 1640$, C III] $\lambda 1908$ and C IV $\lambda 1549$ properties of the galaxies with $\mathrm{EW}(\mathrm{C}$ III $] \lambda 1908)>20 \AA$ in their sample.

At the highest redshifts $(z>6)$, we report in Table 1 the constraints from near-infrared spectroscopy on the restultraviolet emission of three galaxies probing the reionization era. In one of these, the emission-line spectrum from C IV $\lambda 1549$ through CIII] $\lambda 1908$ favours photoionization by stars rather than by an AGN (Schmidt et al. 2017). In another, photoionization could arise from an AGN or hot stars (Stark et al. 2015). In the latter, prominent N V $\lambda 1240$ emission and the low C III] $\lambda 1908 / \mathrm{He}$ II $\lambda 1640$ ratio both support photoionization by an AGN (Laporte et al. 2017). Remarkably, all three galaxies show strong Ly $\alpha$ emission, suggesting intense radiation fields capable of creating early ionized bubbles in the surrounding hydrogen distribution.

Overall, the published spectral analyses of the observations listed in Table 1 consistently point toward galaxies with low metallicities, typically $7.5 \lesssim 12+\log (\mathrm{O} / \mathrm{H}) \lesssim 8.0$, low $\mathrm{C} / \mathrm{O}$ ratios, $-1.0 \lesssim \log \mathrm{C} / \mathrm{O} \lesssim-0.3$, high ionization parameters, $-3.0 \lesssim \log \langle U\rangle \lesssim-1.5$ and large specific star formation rates, from $\sim 10$ to a few $\times 100 \mathrm{Gyr}^{-1}$, across a wide redshift range. The properties of these galaxies approach those expected for primeval galaxies near the reionization epoch (e.g., Stark 2016).

\subsection{LyC leakers}

We are also interested in existing ultraviolet and optical observations of confirmed or potential LyC-leaking galaxies at any redshift. In Table 2, we list the main characteristics of 10 such samples, arranged as before in order of increasing redshift. These include 5 samples of confirmed LyC leakers, in which the restultraviolet emission around $880-912 \AA$ (i.e. just blueward of the Lyman limit) has been directly observed, typically with FUSE and $H S T$ at low redshift and by means of deep optical spectroscopy at higher redshift (Leitet et al. 2011; de Barros et al. 2016; Izotov et al. 2016a,b; Chisholm et al. 2017; Izotov et al. 2018a,b). The reported fractions of escaping LyC photons span a wide range, $1 \lesssim f_{\text {esc }} \lesssim 70$ per cent. As mentioned in Section 2.2 above, this leakage is generally thought to occur either through holes carved into the neutral ISM by extreme galactic outflows or through density-bounded (i.e. optically thin to $\mathrm{LyC}$ photons) 


\begin{tabular}{|c|c|c|c|c|c|c|}
\hline Reference & $z$ & Sample & $12+\log (\mathrm{O} / \mathrm{H})_{\mathrm{gas}}$ & Modelling & Physical parameters $^{a}$ & Comment \\
\hline Leitet et al. (2011) & 0.02 & $\begin{array}{l}\text { FUSE rest- } 900 \AA \text { A detection of } \\
\text { blue compact galaxy Haro } 11\end{array}$ & $\sim 7.9$ & & $f_{\mathrm{esc}} \sim 3 \%$ & $\begin{array}{l}\text { Consistent with 2-dimensional data, count rates, and limits on } \\
\text { residual flux in C II } \lambda \lambda 1036,1037 \text { interstellar absorption line }\end{array}$ \\
\hline Izotov et al. (2017) & $<0.14$ & $\begin{array}{l}5 \text { SDSS compact SF galaxies } \\
\text { with } \mathrm{O}_{32}>20 \text { and no AGN } \\
\text { spectral feature }\end{array}$ & $7.5-7.8$ & $\begin{array}{l}\text { STARBURST99 } \\
\text { CLOUDY } 13.04 \\
\text { (dust physics) }\end{array}$ & $\begin{array}{l}\mathrm{SSFR} \sim 50-400 \mathrm{Gyr}^{-1} \\
N_{\mathrm{H}}<N_{\mathrm{H}}^{\mathrm{IB}} \text { in } 3 \text { galaxies }^{b} \\
\text { (potentially } f_{\text {esc }}>20 \% \text { ) }\end{array}$ & $\begin{array}{l}\mathrm{O}_{32} \text { alone not good indicator of LyC leakage because depends } \\
\text { on details of ionizing spectrum; He I } \lambda 3889, \mathrm{He} \text { I } \lambda 6678 \text { and } \\
\text { He I } \lambda 7065 \text { more promising tracers of density-bounded regions }\end{array}$ \\
\hline Chisholm et al. (2017) & $0.04-0.2$ & $\begin{array}{l}H S T / C O S \text { rest- } 900 \AA \text { archival } \\
\text { data of } 3 \text { confirmed leakers }\end{array}$ & $8.1-8.7$ & & $f_{\text {esc }} \sim 0.4-1.9 \%$ & $\begin{array}{l}\text { Weak Si II } \lambda 1260 \text { and Si III } \lambda 1206 \text { absorption consistent with } \\
\text { density-bounded regions, although gas covering factor may vary }\end{array}$ \\
\hline Jaskot \& Oey (2013) & $0.1-0.2$ & $\begin{array}{l}\text { 6 SDSS Green-Pea galaxies } \\
\text { with } \mathrm{O}_{32}>7 \text { and no AGN } \\
\text { spectral feature } \\
\text { c }\end{array}$ & $7.9-8.0$ & $\begin{array}{l}\text { STARBURST99 } \\
\text { CLOUDY } 10.00 \\
\text { (dust physics) }\end{array}$ & $\begin{array}{l}\text { sSFR } \sim 70-200 \mathrm{Gyr}^{-1} \\
\text { burst age } \sim 3-5 \mathrm{Myr}\end{array}$ & $\begin{array}{l}\text { Large He II } \lambda 1640 / \mathrm{H} \beta \text { ratio not reproducible by standard stellar } \\
\text { population models; if arising from a contribution by shocks, the } \\
\text { associated large } \mathrm{O}_{32} \text { may indicate } N_{\mathrm{H}}<N_{\mathrm{H}}^{\mathrm{IB} b}\end{array}$ \\
\hline Izotov et al. (2016a,b) & $0.3-0.4$ & $\begin{array}{l}H S T / \text { COS rest- } 900 \AA \text { detection } \\
\text { of } 5 \text { compact galaxies with } \\
\mathrm{O}_{32}>5 \text { and no AGN feature }\end{array}$ & $7.8-8.0$ & STARBURST99 & $\begin{array}{l}\text { sSFR } \sim 10-200 \mathrm{Gyr}^{-1} \\
\text { burst age }<4 \mathrm{Myr} \\
\quad f_{\text {esc }} \sim 6-13 \%\end{array}$ & $\begin{array}{l}\text { Compact SF galaxies with large } \mathrm{O}_{32} \text { appear to pick up } \\
\text { efficiently LyC leakers }\end{array}$ \\
\hline Izotov et al. (2018a,b) & $0.3-0.4$ & $\begin{array}{l}H S T / C O S \text { rest- } 900 \AA \text { Aetection } \\
\text { of } 6 \text { compact galaxies with } \\
\mathrm{O}_{32}>8 \text { and no AGN feature }\end{array}$ & $7.6-8.2$ & STARBURST99 & $\begin{array}{l}\text { sSFR } \sim 3-1,000 \mathrm{Gyr}^{-1} \\
\text { burst age } \sim 2-3 \mathrm{Myr} \\
\quad f_{\text {esc }} \sim 2-70 \%\end{array}$ & $\begin{array}{l}\text { General increase of } f_{\text {esc }} \text { with increasing } \mathrm{O}_{32} \text { (and decreasing } \\
\text { velocity spread of Ly } \alpha \text { double-peaked emission) }\end{array}$ \\
\hline Nanayakkara et al. (2019) & 2.2 & $\begin{array}{l}\text { MUSE SF galaxy } 1273 \text {, also } \\
\text { candidate LyC leaker from } \\
\text { Naidu et al. (2017, GS 30668) }\end{array}$ & $\sim 8.3$ & FAST $^{d}$ & $\begin{array}{c}\text { sSFR } \sim 1.5 \mathrm{Gyr}^{-1} \\
\text { (potentially } f_{\mathrm{esc}} \sim 60 \% \text { ) }\end{array}$ & EW(O III $\lambda \lambda 4959,5007) \sim 1200 \AA$ \\
\hline Nakajima et al. (2016) & $3.0-3.7$ & $\begin{array}{l}13 \text { candidate LyC-leaker Ly } \alpha \\
\text { emitters (including } 1 \text { AGN) } \\
\text { and } 2 \text { Lyman-break galaxies }\end{array}$ & $\sim 8.1$ & $\begin{array}{l}\text { STARBURST99 } \\
\text { CLOUDY } 13.02 \\
\text { (dust physics) }\end{array}$ & $\begin{array}{l}\text { SSFR } \sim 1-50 \mathrm{Gyr}^{-1} \\
(\text { Ly } \alpha \text { emitters have } \\
\left.\text { potentially } f_{\text {esc }}>0\right)\end{array}$ & $\begin{array}{l}\text { Ly } \alpha \text { emitters have larger } \mathrm{O}_{32} \text { than Lyman-break galaxies } \\
\text { at similar metallicity, suggesting that the ionized regions } \\
\text { are, at least in part, density bounded }\end{array}$ \\
\hline Vanzella et al. (2016) & 3.1 & $\begin{array}{l}\text { Lensed compact Ly } \alpha \text { emitter } \\
\text { ID11 }\end{array}$ & $<7.8$ & & $f_{\text {esc }}>0$ expected & $\begin{array}{l}\mathrm{O}_{32}>10 \text {, small velocity spread of Ly } \alpha \text { double-peaked emission } \\
\text { and C IV } \lambda 1549 \text { emission suggest low } N_{\mathrm{H}} \text { and LyC leakage }\end{array}$ \\
\hline de Barros et al. (2016) & 3.2 & $\begin{array}{l}\text { VLT/VIMOS rest-900 } \\
\text { detection of candidate LyC } \\
\text { leaker Ion2 }\end{array}$ & $\sim 8.1$ & $\begin{array}{l}\text { POPSTAR } \\
\text { CLOUDY } 13.03 \\
\text { (dust physics) }\end{array}$ & $\begin{array}{l}\mathrm{sSFR} \sim 10 \mathrm{Gyr}^{-1} \\
\log \mathrm{C} / \mathrm{O} \sim-0.8 \\
\quad \mathrm{fesc}_{\mathrm{esc}} \sim 64 \%\end{array}$ & $\begin{array}{l}\text { LyC leakage, Ly } \alpha \text { emission, } \mathrm{O}_{32}>10 \text { and weak } \mathrm{C} \text { II } \lambda 1335 \text { and } \\
\text { Si II } \lambda 1260 \text { absorption suggest density-bounded HII region; } \\
\text { a faint AGN not excluded }\end{array}$ \\
\hline
\end{tabular}

${ }^{a}$ sSFR typically quoted under the assumption $f_{\text {esc }}=0 .{ }^{b} N_{\mathrm{H}}^{\mathrm{IB}}$ is the $\mathrm{H}$-column density required to produce an ionization-bounded nebula. ${ }^{c} \mathrm{O}_{32}=\left[\mathrm{O}_{\mathrm{III}}\right] \lambda 5007 /[\mathrm{O}$ II $] \lambda 3727 .{ }^{d}$ (Kriek et al. 2009). 
HII regions, which are expected to lead to weak low-ionization emission lines, a small velocity spread of the Ly $\alpha$ double-peaked emission and a large [O III] $\lambda 5007 /[\mathrm{O}$ II] $] 33727$ ratio.

The observations reported in Table 2 seem to support the occurrence of enhanced [O III] $\lambda 5007 /[\mathrm{O}$ II $] \lambda 3727$ ratios in confirmed LyC leakers (along with, in some cases, weak lowionization emission lines and a small velocity spread of the Ly $\alpha$ double-peaked profile), which favours density-bounded HiI regions as the main leakage mechanism. In Table 2, we also list 5 samples of candidate LyC leakers, selected on the basis of large observed [O III] $\lambda 5007 /[\mathrm{O}$ II] $\lambda 3727$ ratios, the presence of Ly $\alpha$ emission or deep ultraviolet imaging (Jaskot \& Oey 2013; Nakajima et al. 2016; Vanzella et al. 2016; Izotov et al. 2017; Nanayakkara et al. 2019). Among these studies, Izotov et al. (2017) caution that the $[\mathrm{O} \mathrm{III}] \lambda 5007 /[\mathrm{O} \mathrm{II}] \lambda 3727$ ratio alone is not a certain indicator of LyC leakage, as it depends also on other parameters, such as the ionization parameter, the hardness of ionizing radiation and metallicity. These authors propose an alternative spectral diagnostic of density-bounded Hir regions, based on the He I $\lambda 3889$, He I $\lambda 6678$ and He I $\lambda 7065$ lines.

A comparison between Tables 1 and 2 reveals that LyC leakers have, typically, gas-phase oxygen abundances similar to those of (presumably ionization-bounded) metal-poor star-forming, but specific star formation rates several times larger. The implied extreme radiation fields of these intensively star-forming galaxies likely contribute to the escape of ionizing photons, as suggested by the apparent trend of increasing $f_{\text {esc }}$ with increasing strength of stellar-wind features in the sample of Izotov et al. (2018b).

\subsection{Other star-forming galaxies and AGN}

To complement our reference observational sample, we also include constraints on the ultraviolet and optical nebular emission of star-forming galaxies and AGN at various redshifts from more heterogeneous surveys. In the local Universe, we appeal to the samples of 21 low-metallicity starburst galaxies from Giavalisco et al. (1996), 20 Wolf-Rayet galaxies from López-Sánchez \& Esteban (2008, see also López-Sánchez \& Esteban 2010) and 28 star-forming galaxies from Leitherer et al. (2011, including HII, Seyfert-2 and LINER galaxies). These samples span wide ranges of metallicities, $7.2 \lesssim 12+\log (\mathrm{O} / \mathrm{H}) \lesssim 9.2$. We also gather different samples of more distant galaxies: 9 lensed starforming galaxies at redshifts $z=1.0-3.5$ with optical ( 3 with ultraviolet) emission-line measurements from Christensen et al. (2012, with complementary data from Patrício et al. 2016) and metallicities $7.6 \lesssim 12+\log (\mathrm{O} / \mathrm{H}) \lesssim 8.9$; a composite spectrum of 30 star-forming galaxies with median redshift $z \approx 2.4$ and metallicity $12+\log (\mathrm{O} / \mathrm{H}) \approx 8.14$ from Steidel et al. (2016); 20 Lyman-break galaxies at redshifts $z=3.0-3.8$ from Schenker et al. (2013, we retain 15 galaxies with $\mathrm{H} \beta$ signal-to-noise ratio greater than 2); and 24 Lyman-break galaxies at redshifts $z=3.2-3.7$ from Holden et al. (2016, we retain 19 galaxies with $\mathrm{H} \beta$ signal-to-noise ratio greater than 2). We note that the above samples span wide ranges of metallicities, stellar masses and specific star formation rates, which can overlap in part with those of the samples in Table 1. Finally, we include constraints on the ultraviolet and optical nebular emission of a sample of 12 nearby $(z \lesssim 0.04)$ Seyfert -2 galaxies and 59 radio galaxies and 10 type- 2 (i.e. obscured) quasars at redshifts $1 \lesssim z \lesssim 4$ from Dors et al. (2014, with complementary data from Diaz et al. 1988 and Kraemer et al. 1994), sampling metallicities in the range from roughly 0.1 to 1.0 times solar. We also report the emission-line properties measured by Mignoli et al. (2019) in a composite spectrum of 92 type- 2 AGN in massive galaxies at $1.45<z<3.05$ from the zCOSMOS-deep survey (Lilly et al. 2007).

\subsection{Global observational properties of the full sample}

We plot in Fig. 6 different ultraviolet properties of the full reference sample of metal-poor star-forming galaxies (blue-like colours; see coding at the bottom of Fig. 7), LyC leakers (orangelike colours) and other star-forming galaxies (purple-like colours) and AGN (grey). After exclusion of a few galaxies with incomplete data, the final sample includes 68 metal-poor star-forming galaxies, 16 confirmed and 23 candidate LyC leakers, 75 other star-forming galaxies and 73 AGN. The diagrams in Fig. 6 include several combinations of equivalent widths and ratios of the N v $\lambda 1240$ (hereafter simply N v), C IV $\lambda \lambda 1548,1551$ (hereafter simply C IV), He II $\lambda 1640$, O III] $\lambda \lambda 1661,1666$ (hereafter simply O III]) and [C III] $\lambda 1907+C$ III $] \lambda 1909$ (hereafter simply $\mathrm{C}$ III]) nebular emission lines (and the gas-phase oxygen abundance in Fig. 6a). All but one diagram (Fig. 6g) involve the He II $\lambda 1640$ line, as a main goal of our analysis in the next section will be to assess the specific influence of a comprehensive set of model parameters on predictions of this relative to other line intensities. In Fig. 7, we show the corresponding optical properties of this reference sample, through different ratios involving the $\left[\mathrm{O}_{\text {II }}\right] \lambda \lambda 3726,3729$ (hereafter simply [O II $]$ ), He I $\lambda 3889$, He II $\lambda 4686, \mathrm{H} \beta$, [O III] $\lambda 5007$ (hereafter simply [O III]), $\mathrm{H} \alpha$, [N II] 66584 (hereafter simply [N II]), He I $\lambda 6678$ and He I $\lambda 7065$ nebular emission lines (and the $\mathrm{H} \beta$ equivalent width). All line fluxes in Figs 6 and 7 are corrected for attenuation by dust, as prescribed in the original studies. We note that the line ratios in these figures are subject to uncertainties linked to the different apertures used to observe different galaxy samples, as highand low-ionization lines are not necessarily co-spatial (see, e.g., Kehrig et al. 2018).

The fact that different symbols can populate different diagrams in Figs 6 and 7 illustrate how the many spectral properties we consider are not always available homogeneously for all galaxy samples. This highlights, by itself, the value of the reference sample we have assembled, which allows one to grasp at once the broad ultraviolet and optical properties of the closest known analogues to primeval galaxies, LyC leakers and other star-forming galaxies and AGN. In Fig. 6, over three quarters of all galaxies with ultraviolet data have C III] measurements, sometimes by requirement (Amorín et al. 2017; Nakajima et al. 2018). The line is unavailable for the highest-redshift galaxies, because in part of the limitations affecting ground-based infrared spectroscopy (Schmidt et al. 2017; Stark et al. 2015; Laporte et al. 2017). Many galaxies with C III] measurements also have O III] ones. Over half of all galaxies have He II $\lambda 1640$ and/or C IV measurements, the availability of one line relative to the other being independent of redshift. Finally, in Fig. 6d, Nv is available for only a few galaxies (a young star-forming galaxy with extended Ly $\alpha$ halo from the Christensen et al. 2012 sample, observed by Patrício et al. 2016, which also appears in Figs 6a,b,d and f; the 

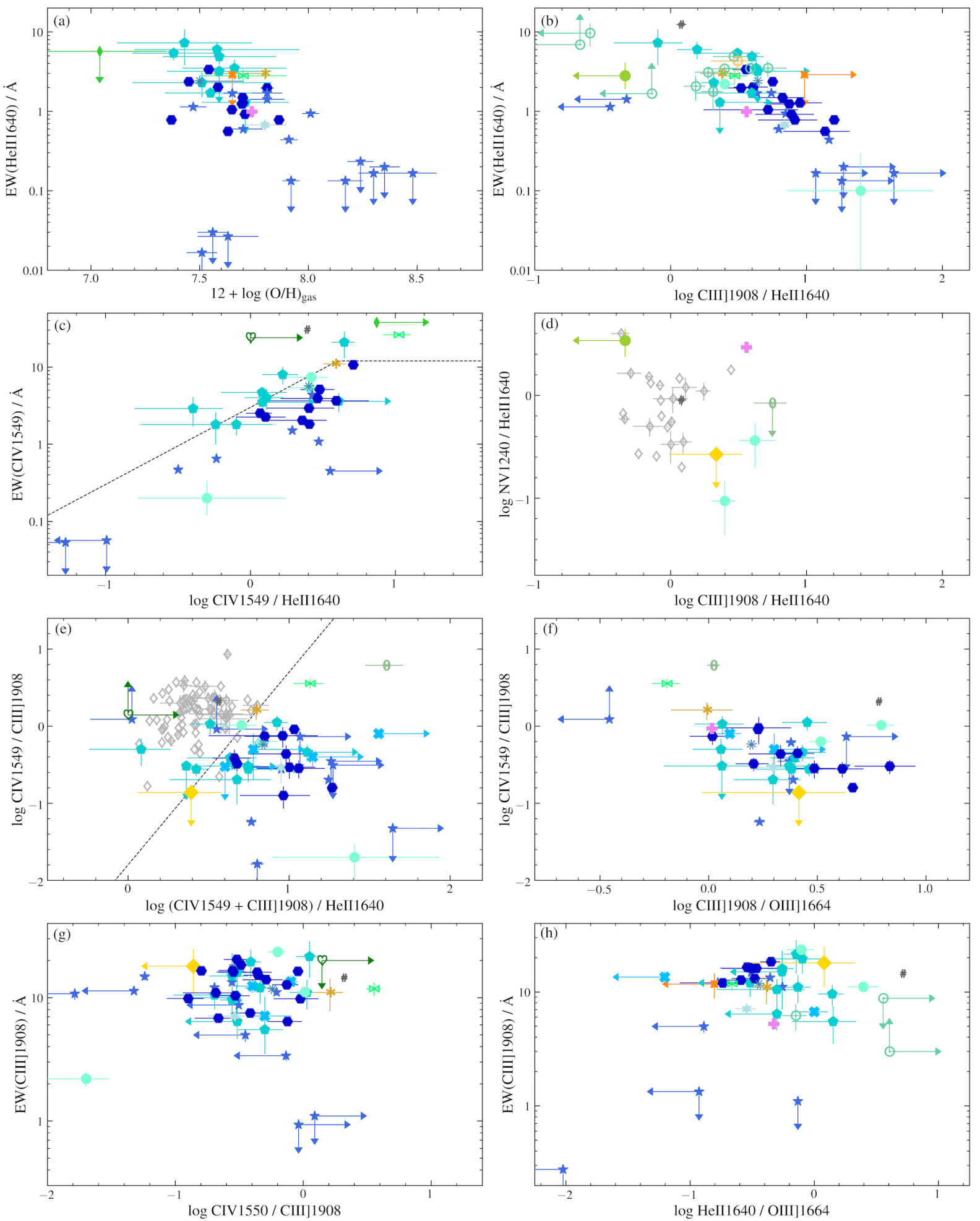

Figure 6. Ultraviolet emission-line properties of the reference observational sample of star-forming galaxies and AGN described in Section 3. Different symbols refer to different samples, as indicated at the bottom of Fig. 7, with blue-like colours corresponding to metal-poor star-forming galaxies, orange-like colours to LyC leakers, purple-like colours to other star-forming galaxies and grey to AGN. The diagrams show different combinations of equivalent widths and ratios of the $\mathrm{N} v \lambda 1240, \mathrm{C}$ IV $\lambda 1549$, He II $\lambda 1640, \mathrm{O}$ III] $\lambda 1664$ and C III] $\lambda 1908$ nebular emission lines [and the gas-phase oxygen abundance in (a)]. In (c) and (e), the dashed lines show the criteria proposed by Nakajima et al. (2018) to separate AGN-dominated from star-forming galaxies. All line fluxes are corrected for attenuation by dust, as prescribed in the original studies. Arrows show $1 \sigma$ upper limits. See description in Section 3.4. 

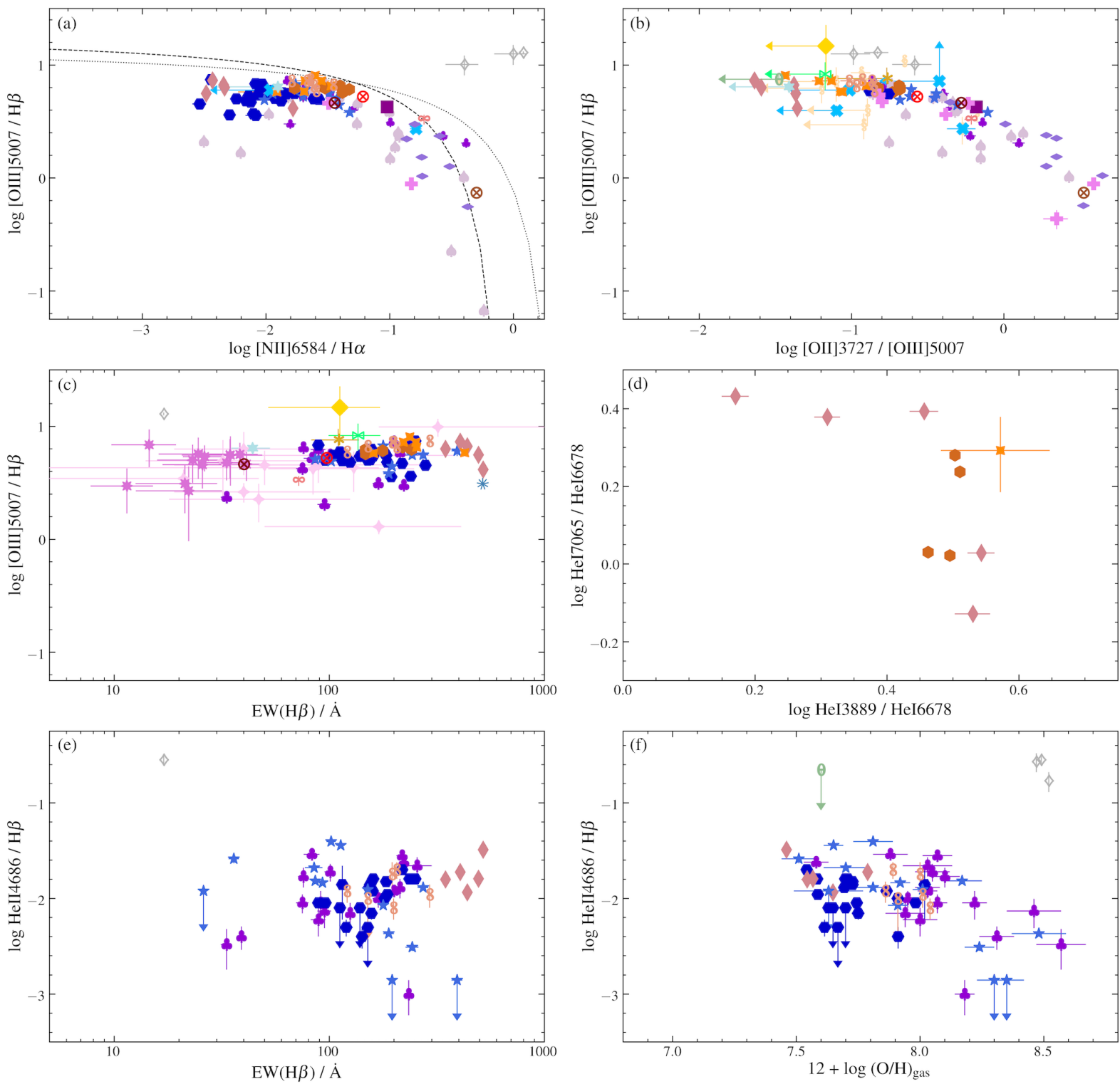

$\begin{array}{ll}\text { - } & \text { Berg+16,19 } \\ \star & \text { Senchyna+17,19 } \\ * & \text { Berg+18 } \\ * & \text { Stark+14 } \\ * & \text { Giavalisco+96 } \\ * & \text { Lopez-Sanchez+10 } \\ \infty & \text { Leitet+11 } \\ \otimes & \text { Izotov+17 } \\ \otimes & \text { Chisholm+17 (Tol-0440-381) } \\ \diamond \quad \text { Dors }+14\end{array}$

$\begin{array}{ll}\text { * } & \text { Erb+10 } \\ & \text { Nakajima+18 }+17 \\ & \\ & \text { Leitherer+11 } \\ \# \quad \text { Christensen+12 } \\ \otimes \quad \text { Chisholm+17 (Tol-1247-232) } \\ 8 \quad \text { Jaskot \& Oey13 } \\ \otimes \quad \text { Chisholm+17 (J0921+4509) } \\ \# \quad \text { Mignoli+19 }\end{array}$

$\begin{array}{llll}\odot & \text { Nanayakkara+19 } & \odot & \text { Schmidt+17 } \\ \bowtie & \text { Vanzella+17 } & & \text { Stark+15 } \\ \theta & \text { Fosbury+03 } & \odot & \text { Laporte+17 } \\ & & & \\ \square & \text { Steidel+16 } & * & \text { Holden+16 } \\ & \text { Schenker+13 } & & \\ - & \text { Izotov+16(a, b) } & & \text { Nakajima+16 } \\ \text { Izotov+18(a, b) } & * & \text { Vanzella+16 } \\ \odot & \text { Nanayakkara+19 } & & \text { De Barros+16 } \\ & & & \end{array}$

Figure 7. Optical emission-line properties of the reference observational sample of star-forming galaxies and AGN described in Section 3 . Different symbols refer to different samples, as indicated at the bottom of the figure, with blue-like colours corresponding to metal-poor star-forming galaxies, orange-like colours to LyC leakers, purple-like colours to other star-forming galaxies and grey to AGN. The diagrams show different ratios of the [O II $] \lambda 3727, \mathrm{He}$ I $\lambda 3889, \mathrm{He} \mathrm{II} \lambda 4686, \mathrm{H} \beta,[\mathrm{O}$ III $] \lambda 5007, \mathrm{H} \alpha,[\mathrm{N} \mathrm{II}] \lambda 6584$, He I $\lambda 6678$ and He I $\lambda 7065$ nebular emission lines (and the $\mathrm{H} \beta$ equivalent width). In (a), the dotted and dashed lines show the criteria of Kewley et al. (2001) and Kauffmann et al. (2003), respectively, to separate AGN-dominated from star-forming galaxies. All line fluxes are corrected for attenuation by dust, as prescribed in the original studies. See description in Section 3.4 . 
Laporte et al. 2017 galaxy; and samples A and B of Nakajima et al. 2018).

The dashed lines in Figs $6 \mathrm{c}$ and $6 \mathrm{e}$ show the criteria proposed by Nakajima et al. (2018) to separate AGN-dominated from star-forming galaxies, based on the He II $\lambda 1640, \mathrm{C} \mathrm{III]}$ and C IV emission lines. Aside from the Dors et al. (2014) AGN sample, only a few galaxies with strong C IV emission lie above the Nakajima et al. (2018) AGN criterion: a few galaxies from the Amorín et al. (2017) sample (although within $2 \sigma$ of the criterion); the Stark et al. (2015) lensed Ly $\alpha$ galaxy, which could be powered by an AGN (Table 1); the Schmidt et al. (2017) lensed Ly $\alpha$ galaxy; in one diagram only (Fig. 6e), the most extreme star-forming galaxy SB 111 in the Senchyna et al. (2017) sample (with an upper limit on C III] emission, accounted for in the horizontal error bar); and, again in one diagram only (Fig. 6c), the Vanzella et al. (2017) lensed double-super star cluster. We note that Schmidt et al. (2017), Senchyna et al. (2017) and Vanzella et al. (2017) find these last three galaxies to be more likely powered by star formation than by an AGN, based on the Gutkin et al. (2016) and Feltre et al. (2016) photoionization models.

A particularly notable feature of Fig. 6 is the trend of increasing He II $\lambda 1640$ equivalent width with decreasing gasphase oxygen abundance (Fig. 6a). High He II $\lambda 1640$ equivalent widths ( $\mathrm{C}$ III]/He II $\lambda 1640$ ratios $(\lesssim 10$; Fig. 6b) and potentially N V emission (according to Fig. 6d). At low EW(He II $\lambda 1640$ ), we find more metal-rich galaxies, with generally lower-ionization gas (e.g., larger C III]/He II $\lambda 1640$ and lower C IV/C III] ratios). This is the case for the few 'normal' star-forming galaxies of our sample with enough ultraviolet data to appear in at least some diagrams of Fig. 6 (e.g.; sample C of Nakajima et al. 2018; one of the Christensen et al. 2012 galaxies, appearing only in Fig. 6h).

Remarkably, galaxies in wide ranges of redshift populate similar regions of the diagrams in Fig. 6, which confirms that modelling low-redshift, metal-poor star-forming galaxies is a useful step toward understanding the physical properties of reionization-era galaxies. A potential shortcoming of such studies is the occurrence at redshifts $z \gtrsim 6$ of C IV equivalent widths well in excess of those found in the nearby Universe (Fig. 6c), which could be attributable to enhanced $\alpha / \mathrm{Fe}$ abundance ratios in high-redshift galaxies (Senchyna et al. 2019). As expected from the comparison between Tables 1 and 2 (Section 3.2), the handful of LyC leakers with available ultraviolet data in our sample tend to overlap with the most extreme star-forming galaxies in Fig. 6 (but notice the low C IV/C III] ratio of the de Barros et al. 2016 galaxy, in which the He II $\lambda 1640$ emission could also be dominated by winds of WR stars; see Vanzella et al. 2019).

This is even more apparent in Fig. 7, as more optical than ultraviolet data are available for LyC leakers and quiescent starforming galaxies in our sample. In Figs $7 \mathrm{a}$ and $7 \mathrm{~b}$, for example, all but a few LyC leakers are concentrated - on top of the general galaxy population - in the high-ionization parts (low [N II $] / \mathrm{H} \alpha$ and $[\mathrm{O} \mathrm{II}] /[\mathrm{O} \mathrm{III}])$ of the standard BPT diagrams defined by the $[\mathrm{O} \mathrm{III}] / \mathrm{H} \beta,[\mathrm{N} \mathrm{II}] / \mathrm{H} \alpha$ and $[\mathrm{O} \mathrm{II}] /[\mathrm{O} \mathrm{III}]$ ratios. In Figs $7 \mathrm{c}, 7 \mathrm{e}$ and $7 \mathrm{f}$, they are concentrated in the regions of highest $\mathrm{H} \beta$ equivalent width, highest $\mathrm{He}$ II $\lambda 4686 / \mathrm{H} \beta$ ratio and lowest gas-phase oxygen abundance. The exceptions are a few weak $\left(f_{\text {esc }} \lesssim\right.$ a few per cent $)$ nearby leakers from Leitet et al. (2011) and Chisholm et al. (2017,
Fig. 7c). The dotted and dashed lines in Fig. 7a show the criteria of Kewley et al. (2001) and Kauffmann et al. (2003), respectively, to separate AGN-dominated from star-forming galaxies. Only the Dors et al. (2014) AGN lie above these lines. Finally, Fig. 7d shows the diagram advertised by Izotov et al. (2017) to diagnose density-bounded Hir regions, based on the He I $\lambda 3889$, He I $\lambda 6678$ and He I $\lambda 7065$ lines (Section 3.2).

\section{EMISSION-LINE SIGNATURES OF GALAXY PHYSICAL PARAMETERS}

In this section, we use the models introduced in Section 2 to investigate the ultraviolet and optical emission-line signatures of a wide range of physical parameters of metal-poor star-forming galaxies in the reference observational diagrams assembled in Section 3. We consider physical parameters pertaining to the interstellar gas, stellar populations, LyC-photon leakage, AGN narrow-line regions and radiative shocks. As described in Section 2, a main attribute of our approach is the adoption of a common parametrization of nebular-gas abundances in all calculations, allowing direct comparisons between models powered by different sources.

To explore the observable signatures of any specific physical parameters in the emission-line diagrams of Figs 6 and 7, it is convenient to examine the offsets implied by changes in this parameter with respect to a 'standard' model with stellar and interstellar properties typical of those expected for young, metal-poor star-forming galaxies. Referring to Table 1, we take this model to correspond to an ionization-bounded galaxy with constant star formation rate $[\psi(t)=$ constant $]$ and the following parameters (see Section 2.1):

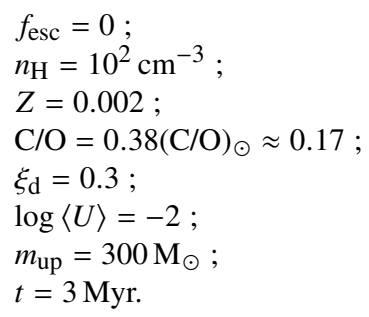

The gas-phase oxygen abundance corresponding to these choices of $Z$ and $\xi_{\mathrm{d}}$ is $12+\log (\mathrm{O} / \mathrm{H})_{\text {gas }} \approx 7.83$ (see table 1 of Gutkin et al. 2016). In this standard model, we do not include interstellar-line absorption in the HII interiors and $\mathrm{H}_{\mathrm{I}}$ envelopes of stellar birth clouds, nor any contribution by an AGN or radiative shocks to nebular emission. We compute the emission-line properties of the model using the $\mathrm{C} \& \mathrm{~B}$ stellar population synthesis code.

Figs 8 and 9 show the ultraviolet and optical emission-line properties of the standard model (black circle) in the same diagrams as in Figs 6 and 7, where all the observations have been greyed for clarity. Also shown are a more metal-poor model with $Z=0.0005$ (black upside-down triangle) and a more metal-rich one with $Z=0.008$ (black square), with all other parameters fixed. Figs 10 and 11 show the standard model again, along with a lower-ionization model with $\log \langle U\rangle=-3$ (small black 
circle) and a higher-ionization one with $\log \langle U\rangle=-1$ (large black circle), at fixed other parameters. Overall, Figs 8-11 indicate that these five models sample reasonably well the observed ultraviolet and optical emission-line properties of metal-poor, actively star-forming galaxies [i.e., with $[\mathrm{N}$ II] $/ \mathrm{H} \alpha \lesssim 0.1$ and $\mathrm{EW}(\mathrm{H} \beta) \gtrsim 200 \AA$ in Figs 9 and 11], except in diagrams involving the He II $\lambda 1640$ and He II $\lambda 4686$ lines. In such diagrams, the data for low oxygen abundances $\left[12+\log (\mathrm{O} / \mathrm{H})_{\text {gas }} \lesssim 8.0\right.$; see Figs $8 \mathrm{a}, 9 \mathrm{f}, 10 \mathrm{a}$ and 11f] tend to exhibit much stronger He II emission than predicted by models with classical stellar populations, as pointed out in several previous studies (Section 1). The five benchmark models also do not quite reach the highest observed equivalent widths of C IV ( C III] ( $20 \AA$; e.g., Figs $8 \mathrm{~g}$ and $10 \mathrm{~g}$ ), nor the highest C III]/O III] ratios ( $\gtrsim 3$; Figs $8 \mathrm{f}$ and 10f).

We now examine the observable signatures of each adjustable parameter of the models in the emission-line diagrams of Figs $8-11$. We describe the effects of altering a single parameter at a time, keeping all other parameters fixed:

Metallicity, $Z$. Increasing metallicity from $Z=0.0005$ (black upside-down triangle), to 0.002 (circle), to 0.008 (square) in Figs 8 and 9 raises cooling through collisionally-excited metal transitions, causing the electronic temperature, $T_{\mathrm{e}}$, to drop. The luminosity ratios of metal-to- $\mathrm{H}$ and $\mathrm{He}$ lines tend to increase at first, and then drop when $T_{\mathrm{e}}$ is low enough for cooling to shift from ultraviolet and optical to infrared transitions (e.g. Spitzer 1978). This is particularly visible for C III]/He II $\lambda 1640$ [Fig. 8b; note also the behaviour of EW(C III]) in Fig. 8g], while for [O III]/H $\beta$ (Fig. 9a) the maximum is reached around $Z \approx 0.006$ (see fig. 2 of Gutkin et al. 2016). In contrast, N V/He II $\lambda 1640$ (Fig. 8d) and $[\mathrm{N} \mathrm{II}] / \mathrm{H} \alpha$ (Fig. 9a) keep rising as $Z$ increases, because of the inclusion of secondary nitrogen production in our model. Meanwhile, He I $\lambda 3889 / \mathrm{He}$ I $\lambda 6678$ and He I $\lambda 7065 / \mathrm{He}$ I $\lambda 6678$ both decrease as $Z$ rises and $T_{\mathrm{e}}$ declines (Izotov et al. 2017). At fixed ionization parameter, a rise in $Z$ also implies lower ratios of high- to low-ionization lines, such as smaller C IV/C III] [Fig. 8c; note also the behaviour of EW(C IV) in Fig. 8c] and larger $\mathrm{C} \mathrm{III]/O} \mathrm{III]} \mathrm{(Fig.} \mathrm{8f)} \mathrm{and} \mathrm{[O} \mathrm{II]/[O} \mathrm{III]} \mathrm{(Fig.} \mathrm{9b),} \mathrm{because}$ the inner high-ionization parts of HiI regions shrink (e.g., Fig. 3 above; see also Stasińska 1980).

The behaviour of the recombination-line ratio $\mathrm{He}$ II $\lambda 4686 / \mathrm{H} \beta$ in Fig. 9e is linked to the evolution of very massive stars ( $>100 \mathrm{M}_{\odot}$ ) in the models. As $Z$ increases, the competing effects of the weakening of ionizing radiation caused by the lower effective temperatures of metal-rich relative to metal-poor stars (e.g., fig. 15 of Bressan et al. 2012) and the hardening of ionizing radiation caused by an increase in mass-loss rate (e.g., Vink et al. 2001; Crowther 2006) conspire in making $\mathrm{He}$ II $\lambda 4686 / \mathrm{H} \beta$ larger at $Z=0.002$ than at 0.0005 and 0.008 (see also Fig. 12 of Section 5; we note that this is not the case for $m_{\text {up }}=100 \mathrm{M}_{\odot}$; see below). The rise in $\operatorname{EW}(\mathrm{H} \beta)$ as $Z$ increases in this diagram results from the slower evolution of metal-rich relative to metalpoor stars, delaying the appearance of evolved stars with strong optical continua.

Zero-age volume-averaged ionisation parameter, $\langle U\rangle$. Increasing $\log \langle U\rangle$ from -3 (small black circle), to -2 (mediumsize circle), to -1 (large circle) in Figs 10 and 11, which can be achieved in our model by raising the gas-filling factor $\epsilon$ at fixed ionizing-photon rate $Q$ and H-density $n_{\mathrm{H}}$ (equation 4), increases the probability of multiple ionization. This causes $\mathrm{N}$ V/He II $\lambda 1640$ (Fig. 10d), C IV/C III] (Fig. 10e), [O III]/H $\beta$ (Fig. 11a), [O III]/[O II] (inverse abscissa of Fig. 11b) and He II $\lambda 4686 / \mathrm{H} \beta$ (Fig. 11f) to rise. The equivalent widths of He II $\lambda 1640$ (Fig. 10a), C IV (Fig. 10c) and C III] (Fig. 10h) also increase. Instead, C III]/He II $\lambda 1640$ (Fig. 10b), C IV/He II $\lambda 1640$ (Fig. 10c) and $\mathrm{OIII} / \mathrm{He}$ II $\lambda 1640$ (inverse abscissa of Fig. 10h) first increase, and then decrease when $\langle U\rangle$ rises. This is because while the He II $\lambda 1640$ luminosity continues to rise, the rise of $\mathrm{C}$ III], $\mathrm{C}$ IV and $\mathrm{O}$ III] is slowed down by the conversion of $\mathrm{C}^{+}$into $\mathrm{C}^{2+}, \mathrm{C}^{2+}$ into $\mathrm{C}^{3+}$ and $\mathrm{O}^{2+}$ into $\mathrm{O}^{3+}$. Similarly, $[\mathrm{N}$ II $] \lambda 6584 / \mathrm{H} \alpha$ drops (Fig. 11a) because of the conversion of $\mathrm{N}^{+}$into $\mathrm{N}^{2+}$.

Increasing $\langle U\rangle$ at fixed other parameters also makes the $\mathrm{H}$ column density, and hence the dust optical depth, larger (Section 2.2). The enhanced absorption of ionizing photons by dust is the reason for the drop in $\operatorname{EW}(\mathrm{H} \beta)$ in Fig. 11e. Since the grain opacity peaks near $912 \AA$ and declines toward shorter wavelengths (e.g., Bottorff et al. 1998), He II $\lambda 4686$-ionizing photons are less absorbed than $\mathrm{H}$-ionizing ones, an effect amplified by the fact that the $\mathrm{H} \beta$ line is produced further out in HII regions than the He II $\lambda 4686$ line. This effect contributes to the rise in $\mathrm{He}$ II $\lambda 4686 / \mathrm{H} \beta$ from $\log \langle U\rangle=-3$ to -1 in this diagram (see also Erb et al. 2010). Also, the associated increase in He I-column density amplifies the effects of fluorescence, causing $\mathrm{He}$ I $\lambda 3889 / \mathrm{He}$ I $\lambda 6678$ to drop and $\mathrm{He}$ I $\lambda 7065 / \mathrm{He}$ I $\lambda 6678$ to rise (Fig. 11d; see Izotov \& Thuan 1998).

Carbon-to-oxygen abundance ratio. Increasing the $\mathrm{C} / \mathrm{O}$ ratio from 0.17 to $(\mathrm{C} / \mathrm{O})_{\odot}=0.44$ (blue segments in Figs $\left.8-11\right)$ is achieved in our model by raising the carbon abundance and lowering the abundances of all other metallic elements - including oxygen - at fixed total metallicity $Z$ (see section 2.3 .1 of Gutkin et al. 2016). This makes the C III] $\lambda 1908$ and $\mathrm{C}$ IV $\lambda 1549$ lines stronger (Figs 8 and 10) and the [O II], [O III] and [N II] lines slightly weaker (Figs 9 and 11), while the $\mathrm{H}$ and $\mathrm{He}$ lines are negligibly affected (Figs 9d-f and 11d-f ).

Dust-to-metal mass ratio, $\xi_{\mathrm{d}}$. Lowering the dust-to-mass ratio from $\xi_{\mathrm{d}}=0.3$ to 0.1 (dark-green segments in Figs 8-11) increases the abundance of coolants in the gas phase. This causes line luminosities from the most abundant refractory coolants (such as $\mathrm{O}$ and $\mathrm{C}$ ) to rise, while at the same time, the drop in electronic temperature reduces cooling through ultraviolet and optical transitions, an effect which becomes dominant at high metallicity. Hence, EW(C III]) (Fig. 8g), EW(C IV) (Fig. 8c), $\mathrm{C} \mathrm{III]/He} \mathrm{II} \lambda 1640$ (Fig. 8b) and [O III]/H $\alpha$ (Fig. 9a) rise at low $Z$ but drop at high $Z$ when $\xi_{\text {d }}$ declines from 0.3 to 0.1. In Fig. 8f, $\mathrm{C} \mathrm{III]/O} \mathrm{III]} \mathrm{increases} \mathrm{because} \mathrm{carbon} \mathrm{is} \mathrm{more} \mathrm{depleted} \mathrm{than} \mathrm{oxy-}$ gen (see, e.g., table 1 of Gutkin et al. 2016). Lowering $\xi_{\mathrm{d}}$ also makes the dust optical depth smaller, causing $\mathrm{He}$ II $\lambda 4686 / \mathrm{H} \beta$ to drop and $\mathrm{EW}(\mathrm{H} \beta)$ to rise in Figs 9e and 11e (see discussion of $\langle U\rangle$ above). We note that the dust optical depth is not simply proportional to the product $\xi_{\mathrm{d}} Z$, as absorption of ionizing photons by dust when $Z$ increases also reduces the HiI-region radius, and hence $N_{\mathrm{H}}$ (equation 8 and Fig. 2).

Hydrogen gas density, $n_{\mathrm{H}}$. Increasing $n_{\mathrm{H}}$ from $10^{2}$ to $10^{3} \mathrm{~cm}^{-3}$ (yellow segments in Figs 8-11) enhances collisional excitation, but also favours collisional over radiative de-excitation of excited species. The cooling through infrared transitions is reduced and that through ultraviolet and optical transitions enhanced, because the critical density for collisional de-excitation is lower for infrared fine-structure than for ultraviolet and optical transitions. The effect is most visible at high metallic- 

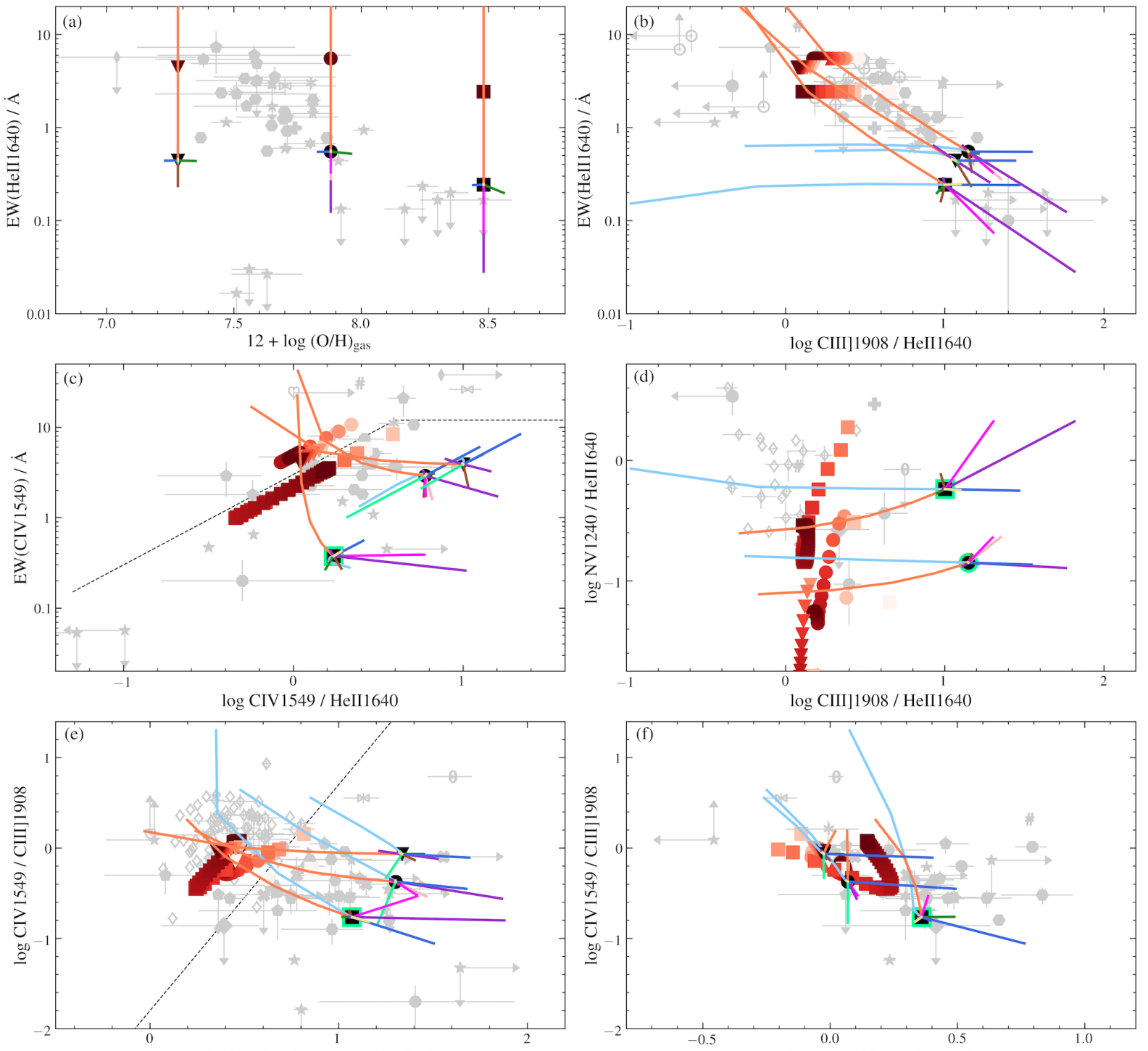

$\log (\mathrm{CIV} 1549+\mathrm{CIII}] 1908) / \mathrm{HeII} 1640$
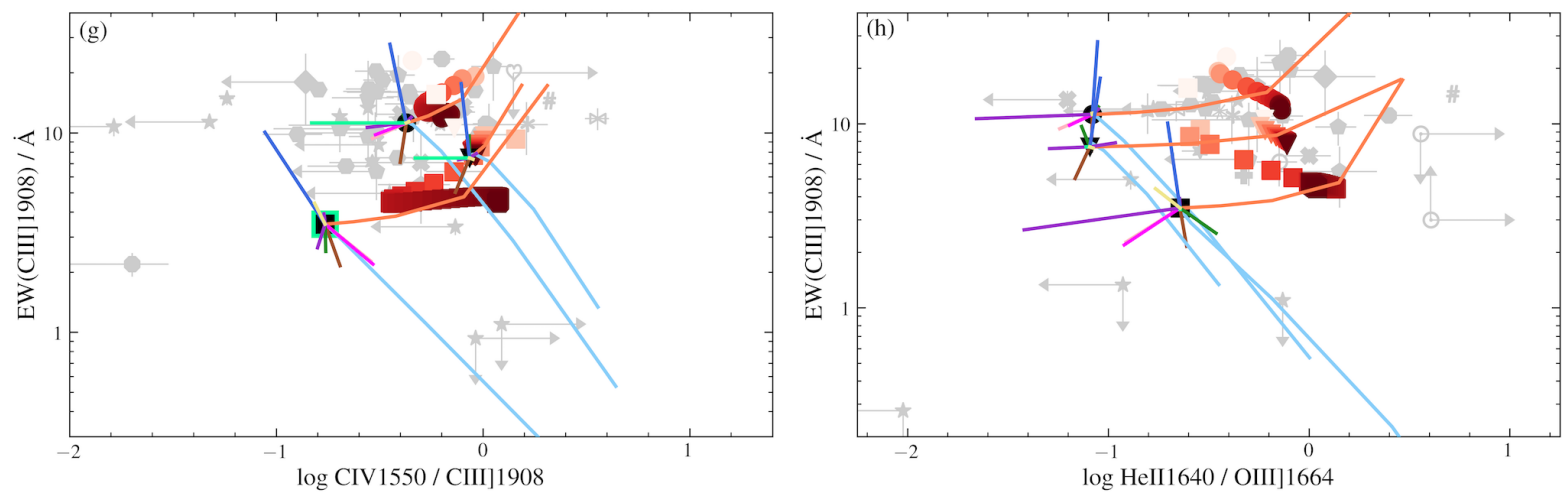

Figure 8. Same diagrams as in Fig. 6, but where the observations have been greyed for clarity. The black circle corresponds to the 'standard' model (with metallicity $Z=0.002$ and $\log \langle U\rangle=-2$ ) described in Section 4, while the black upside-down triangle and black square are benchmark models with the same parameters, but with $Z=0.0005$ and 0.008 , respectively. Segments of different colours show the effect of altering a single parameter at the time (as summarized at the bottom of Fig. 9): rise in $\mathrm{C} / \mathrm{O}$ ratio from 0.17 to $(\mathrm{C} / \mathrm{O})_{\odot}=0.44$ (blue); drop in dust-to-mass ratio from $\xi_{\mathrm{d}}=0.3$ to 0.1 (dark green); rise in $n_{\mathrm{H}}$ from $10^{2}$ to $10^{3} \mathrm{~cm}^{-3}$ (yellow); inclusion of interstellar-line absorption in the HII interiors and outer Hi envelopes of stellar birth clouds (light green); increase in stellar population age from 3 to $10 \mathrm{Myr}$ (brown); rise in $m_{\text {up }}$ from 100 , to 300 , to $600 \mathrm{M}_{\odot}$ (dark purple); adopting the BPASS single- (light purple) and binary-star (magenta) models in place of the C\&B model (BPASS models are not available for $Z=0.0005$ ); drop in the optical depth $\tau_{570}$ from +1.0 to -1.0 (light blue); inclusion of an AGN component contributing from 0 to 99 per cent of the total He II $\lambda 1640$ emission (orange); and inclusion of a radiative-shock component contributing 90 per cent of the total He II $\lambda 1640$ emission [red symbols, with shape corresponding to the metallicity of the associated benchmark model, and darkness to the shock velocity, from $10^{2} \mathrm{~km} \mathrm{~s}^{-1}$ (light) to $10^{3} \mathrm{~km} \mathrm{~s}{ }^{-1}$ (dark)]. 

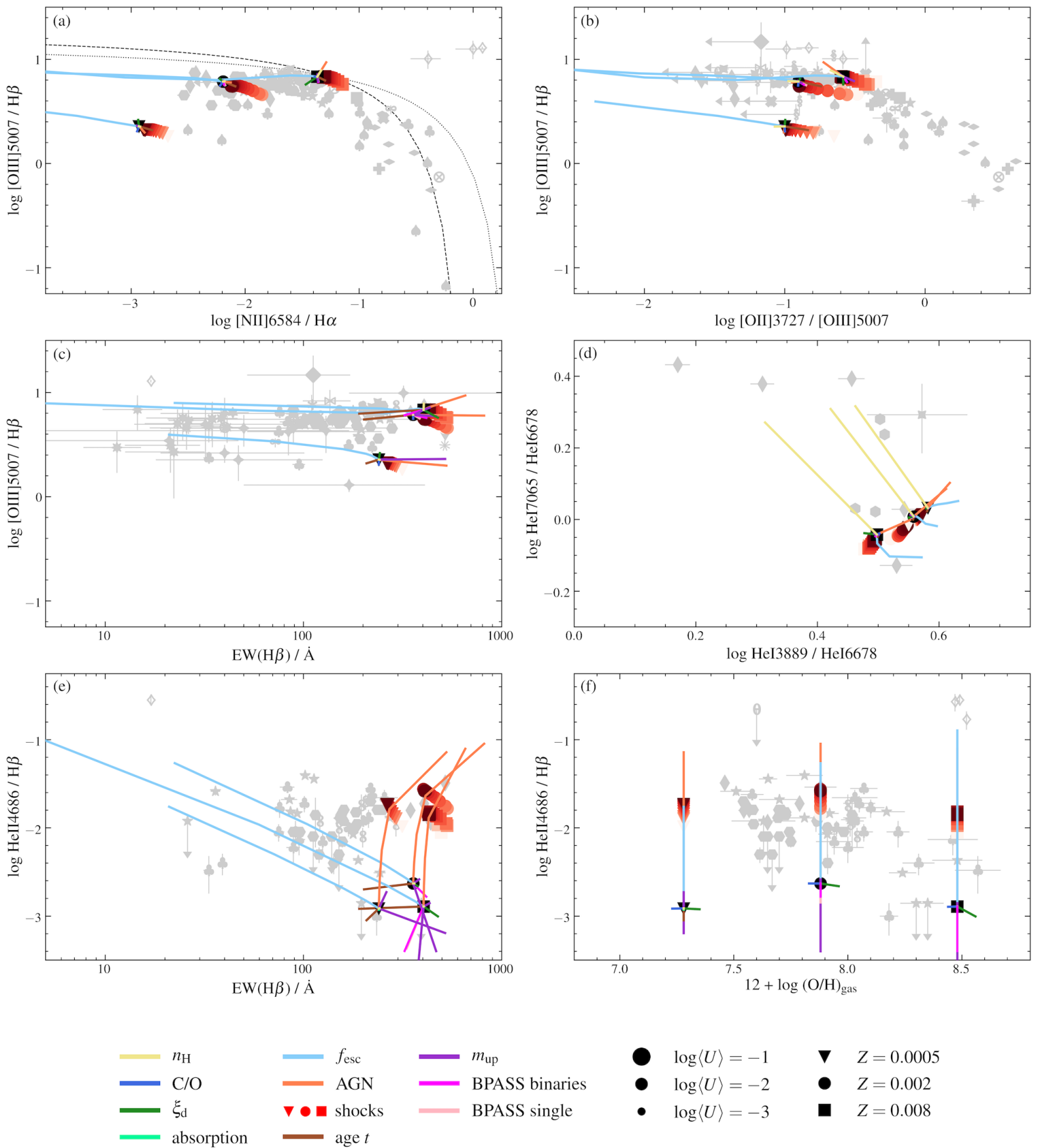

Figure 9. Same diagrams as in Fig. 7, but where the observations have been greyed for clarity. The models are the same as in Fig. 8 .

ity, where infrared transitions tend to dominate the cooling (e.g., Oey \& Kennicutt 1993). Thus, EW(C III]) (Fig. 8g), C III]/He II $\lambda 1640$ (Fig. 8b), EW(C IV) and C IV/He II $\lambda 1640$ (Fig. 8c), O III]/He II $\lambda 1640$ (inverse abscissa of Fig. 8h) and $[\mathrm{N} \mathrm{II}] / \mathrm{H} \alpha$ and $[\mathrm{O} \mathrm{III}] / \mathrm{H} \beta$ (Fig. 9a) rise together with $n_{\mathrm{H}}$. Since increasing $n_{\mathrm{H}}$ at fixed other parameters in our model implies reducing the gas-filling factor as $\epsilon \propto 1 / \sqrt{n_{\mathrm{H}}}$ (equation 4), this causes the dust optical depth to rise as $\sqrt{n_{\mathrm{H}}}$ (equation 8 ), the effect of which is nonetheless subtle in Figs 8-11. In contrast, the He I lines (Figs 9d and 11d) are quite sensitive to changes in $n_{\mathrm{H}}$. This is because the $\lambda 7065$ transition is much more respon- sive to collisional enhancement than the $\lambda 6678$ transition, itself more so than the $\lambda 3889$ transition (Izotov et al. 2017), causing $\mathrm{He}$ I $\lambda 3889 / \mathrm{He}$ I $\lambda 6678$ to drop and $\mathrm{He}$ I $\lambda 7065 / \mathrm{He}$ I $\lambda 6678$ to rise significantly as $n_{\mathrm{H}}$ increases.

Interstellar-line absorption. The light-green segments in Figs 8-11 show the effect of accounting for interstellar-line absorption in the Hı interiors and outer Hi envelopes of stellar birth clouds, following the prescription of Vidal-García et al. (2017, see Section 2.1 above). As expected, the effect is most striking for the C IV $\lambda 1549$ and NV $\lambda 1240$ resonance lines, whose net emission can be drastically reduced and even entirely canceled 

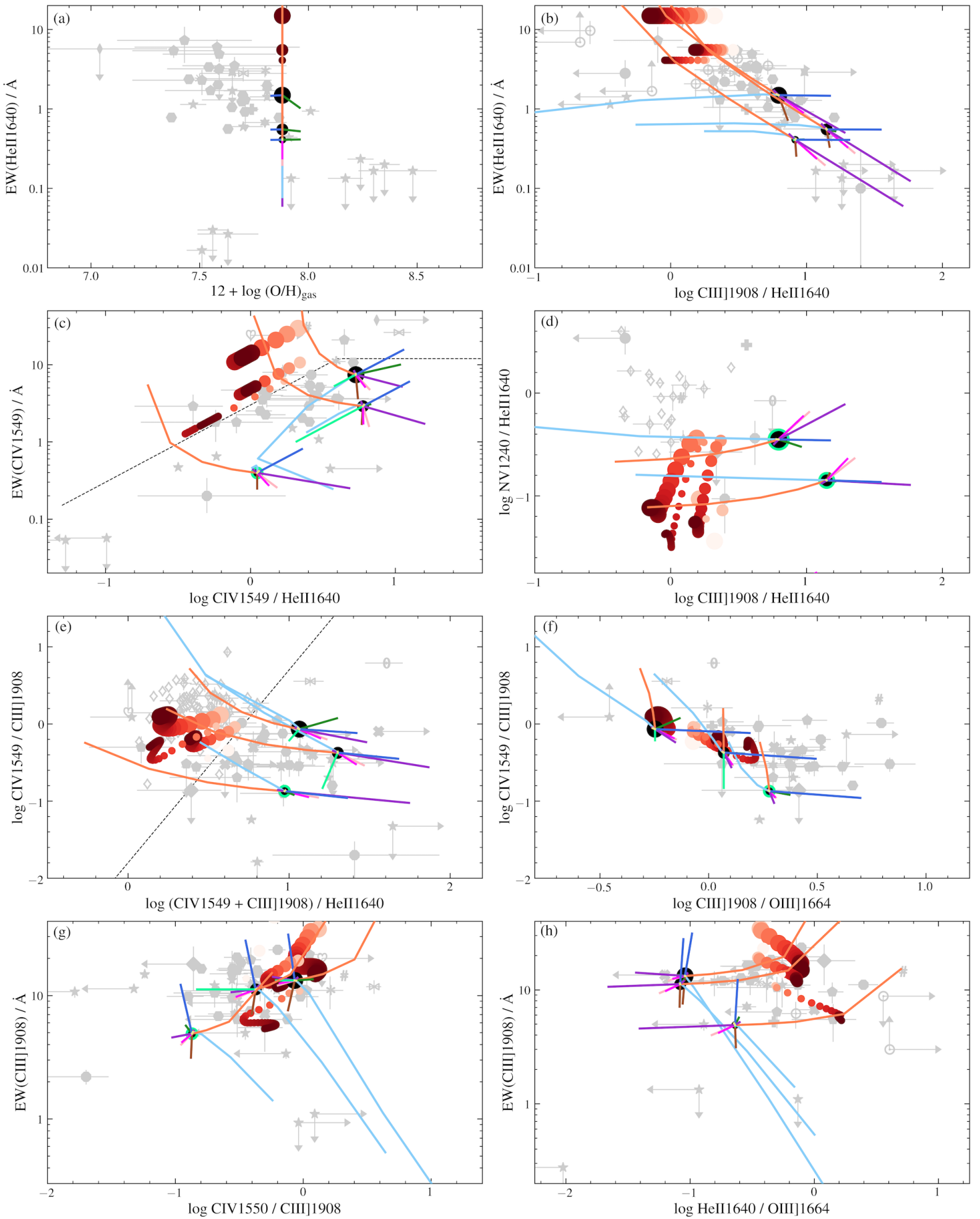

Figure 10. Same as Fig. 8, but for models with the metallicity $Z=0.002$ only, and for three values of the zero-age volume-averaged ionisation parameter, $\log \langle U\rangle=-3,-2$ and -1 (in order of increasing symbol size). 

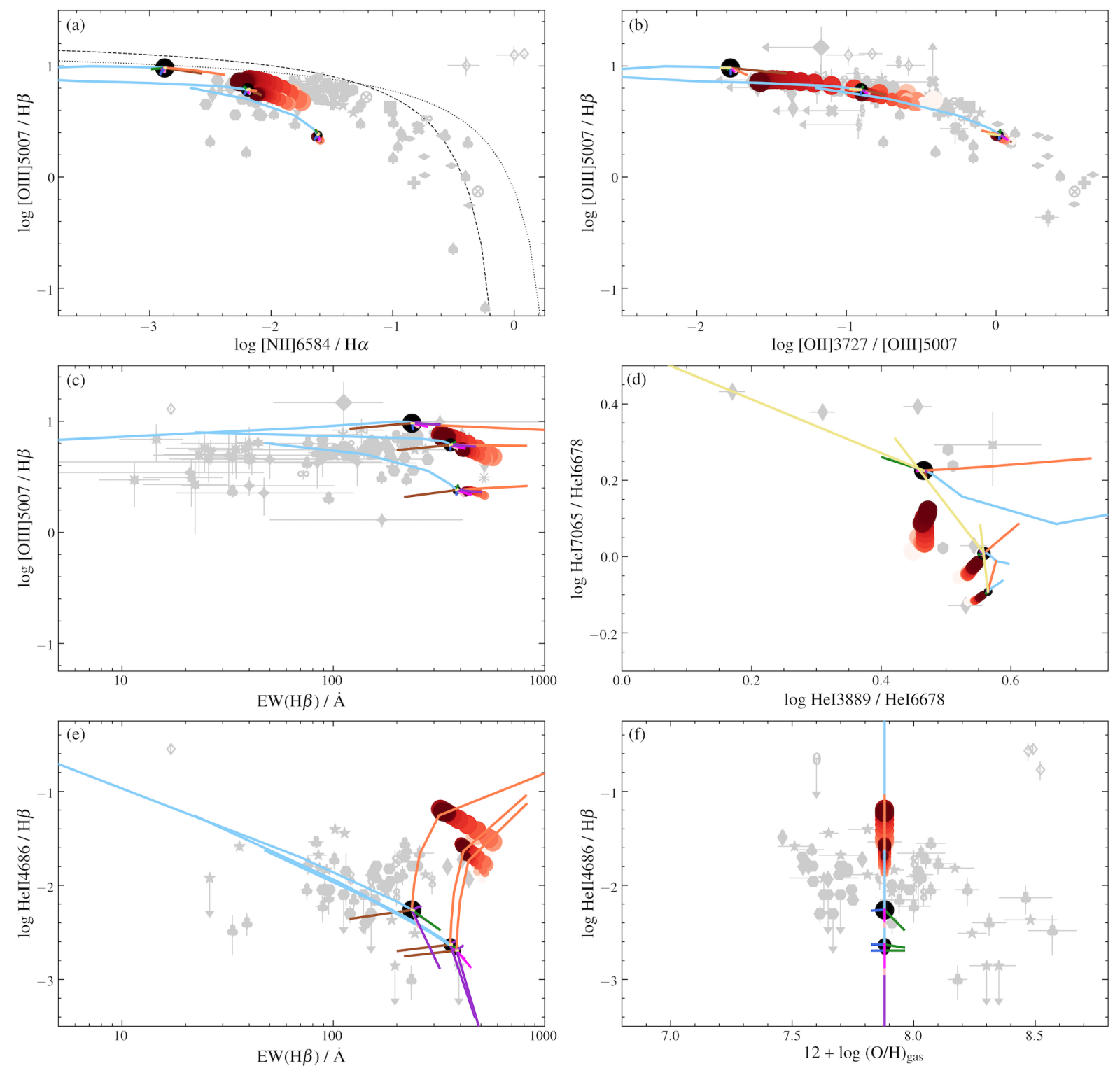

Figure 11. Same as Fig. 9, but for the same models as in Fig. 10.

- as pictured by light-green aureolas around some benchmark models - in Figs 8 and 10.

Stellar population age, $t$. At constant star formation rate, the age of the stellar population sets the age of the oldest HII regions contributing to the nebular emission from a galaxy in our model (equation 1). In an individual Нı region, the rate of ionizing photons, and hence, the ionization parameter (equation 4), drop sharply at ages after about 3 Myr (e.g., Fig. 1d). For $\psi(t)=$ constant, therefore, the global effective ionization parameter of the population of Hir regions declines until a stationary population of ionizing stars is reached, which happens around $t=10 \mathrm{Myr}$ in the $\mathrm{C} \& \mathrm{~B}$ models (but see also Section 5.1 below). Thus, increasing $t$ from 3 to $10 \mathrm{Myr}$ (brown segments in Figs 8-11) tends to have an effect similar on emission lines to that of decreasing $\langle U\rangle$ (see above), such as making [N II]/H $\alpha$ (Figs 9a and 11a) and [O II ]/[O III] (Figs 9b and 11b) larger and $\mathrm{He}$ II $\lambda 4686 / \mathrm{H} \beta$ (Figs 9e and 11e) smaller. The equivalent widths of He II $\lambda 1640$ (Figs 8b and 10b), C IV (Figs 8c and 10c) and C III] (Fig. 8g and 10g) drop because of the build-up of continuum flux from older stellar populations.

Upper mass cut-off of the IMF, $m_{\mathrm{up}}$. At fixed $\langle U\rangle$ and other parameters, increasing $m_{\text {up }}$ from 100 , to 300 , to $600 \mathrm{M}_{\odot}$ (darkpurple segments in Figs 8-11) hardens the ionizing spectrum, because massive stars evolve at higher temperatures than lowermass stars. The effect is much stronger from 100 to $300 \mathrm{M}_{\odot}$ than from 300 to $600 \mathrm{M}_{\odot}$, because of the upturn of the upper main sequence in the Hertzsprung-Russell diagram. The hardening of the spectrum primarily implies larger ratios of He II-to-other lines, such as He II $\lambda 1640 / \mathrm{O} \mathrm{III]} \mathrm{(Figs} \mathrm{8h} \mathrm{and} \mathrm{10h)} \mathrm{and} \mathrm{He} \mathrm{II} \lambda 4686 / \mathrm{H} \beta$ (Figs 9e and 11e), and in turn, smaller C III]/He II $\lambda 1640$ (Figs 8b and $10 \mathrm{~b}$ ) and CIV/He II $\lambda 1640$ (Figs $8 \mathrm{c}$ and $10 \mathrm{c}$ ). We note that, for $m_{\mathrm{up}}=100 \mathrm{M}_{\odot}$, He II $\lambda 4686 / \mathrm{H} \beta$ increases steadily from $Z=0.008$, to 0.002 , to 0.0005 (Fig. 9e), because of the higher effective temperatures of metal-poor relative to metal-rich stars 
(see above). Raising $m_{\text {up }}$ also strengthens the equivalent width of He II $\lambda 1640$ (Figs 8a and 10a), and to a lesser extent, those of C IV (Figs 8c and 10c) and C III] (Figs 8g and 10g). Finally, we note that, for $m_{\text {up }}=600 \mathrm{M}_{\odot}$, a stellar He II $\lambda 1640$ wind feature can arise even at the metallicity $Z=0.0005$ in the models of Figs $8-11$ (with predicted equivalent width $\sim 1.7 \AA$ and full width at half-maximum $\sim 1800 \mathrm{~km} \mathrm{~s}^{-1}$ ).

Stellar population synthesis model. The light-purple and magenta segments in Figs 8-11 show the effect of using the BPASS single- and binary-star models, respectively, in place of the C\&B model, to compute $S_{\lambda}\left(t^{\prime}\right)$ in equation (1). At the considered age of $3 \mathrm{Myr}$, both versions of the BPASS model tend to produce slightly softer ionizing radiation than the C\&B model, which incorporates recent evolutionary tracks and model atmospheres for massive stars (Section 2.1; see also Section 5.1 below). As a result, in all panels of Figs 8-11, changing from the C\&B to BPASS models has an effect on line ratios and equivalent widths similar to that of lowering $m_{\text {up }}$ (see above). ${ }^{4}$ As expected, the BPASS binary-star model produces harder radiation than the BPASS single-star model (e.g., Stanway \& Eldridge 2019), the effect increasing toward later ages (not shown). It is also worth noting that, since a majority of massive stars are expected to undergo binary interactions (e.g., Sana et al. 2012), the He IIline strengths predicted by the single-star C\&B models should be considered as lower limits.

Fraction of escaping LyC photons, $f_{\text {esc }}$. The light-blue segments in Figs 8-11 show the effect of decreasing $\tau_{570}$, the zeroage optical depth of Hit regions to LyC photons with wavelength $\lambda=570 \AA$ (Section 2.2), from +1.0 to -1.0 . This is equivalent to increasing $f_{\text {esc }}$ from zero to nearly unity (Fig. 1c). As seen in Section 2.2 (Figs 3 and 4), increasing $f_{\text {esc }}$ progressively removes the outer low-ionization zones of HII regions, causing C IV/C III] (Figs $8 \mathrm{c}$ and 10c) and [O III]/[O II] (inverse abscissa of Figs 9b and $11 \mathrm{~b}$ ) to rise, while $\mathrm{C}$ III]/He II $\lambda 1640$ (Figs $8 \mathrm{~b}$ and 10b) and the equivalent widths of $\mathrm{C} \mathrm{III]} \mathrm{(Figs} 8 \mathrm{~g}$ and $10 \mathrm{~g}$ ) and $\mathrm{H} \beta$ (Figs 9e and 11e) drop sharply (at ages $t>3 \mathrm{Myr}$ for large $f_{\text {esc }}$, the effect on [O III]/[O II] would be inverted; see Fig. 5f). Also, as noted by Izotov et al. (2017), increasing $f_{\text {esc }}$ makes He I $\lambda 3889 / \mathrm{He}$ I $\lambda 6678$ larger because of the high sensitivity of the $\lambda 3889$ transition to fluorescence (which increases the line luminosity as the optical depth decreases), while the effect on He I $\lambda 7065 / \mathrm{He}$ I $\lambda 6678$ is weaker (Figs 9d and 11d). This led Izotov et al. (2017) to argue that $\mathrm{He}$ I lines could be a promising alternative to [O III $] /[\mathrm{O}$ II $]$ to constrain $f_{\text {esc }}$ in star-forming galaxies.

AGN component. The orange segments in Figs 8-11 show the effect of adding an AGN component contributing from 0 to 99 per cent of the total He II $\lambda 1640$ emission, using the prescription of Section 2.3 (this corresponds roughly to a contribution from 0 to $40-80$ per cent of the total $\mathrm{H} \beta$ emission, depending on the model). In these Seyfert 2-galaxy models, the AGN narrowline region contributes to the nebular (line and recombinationcontinuum) emission, but not the underlying ultraviolet and optical emission. Thus, the equivalent widths of He II $\lambda 1640$ (Figs 8a

4 The photoionization modelling in the comparison of $\mathrm{C} \& \mathrm{~B}$ and BPASS v2.2.1 models presented in Figs $8-11$ is fully self-consistent and includes dust physics. We note that Xiao et al. (2018) compare the Gutkin et al. (2016) models, which include dust physics, with dust-free photoionization models computed using BPASS v2.1 (Xiao et al. 2018 also inadvertently plotted C IV/He II $\lambda 1640$ in place of C III]/He II $\lambda 1640$ from the Gutkin et al. 2016 models in their fig. B1; E. Stanway, private communication). and 10a), C IV (Figs 8c and 10c), C III] (Figs 8h and 10h) and $\mathrm{H} \beta$ (Figs 9e and 11e) all rise. The much harder spectra of AGN relative to stars at high energies (e.g., fig. 1 of Feltre et al. 2016) imply larger ratios of He II-to-other lines and larger C IV/C III] (Figs $8 \mathrm{e}$ and 10e) and [O III]/[O II] (inverse abscissa of Figs 9b and 11b), the AGN component accounting for nearly all the He II $\lambda 1640$ emission in the most extreme models. We note that $\mathrm{N}$ V/He II $\lambda 1640$ drops in Figs 8d and 10d, because of the conversion of $\mathrm{N}^{4+}$ into $\mathrm{N}^{5+}$. In these figures, the Dors et al. (2014) and Mignoli et al. (2019) observations of powerful AGN hosted by massive galaxies can be reproduced by models with metallicity $Z \approx 0.008$ and high ionization parameters, $-2 \lesssim \log \langle U\rangle \lesssim-1$ (see also section 4.2 of Mignoli et al. 2019). Finally, the larger $n_{\mathrm{H}}$ of the AGN models $\left(10^{3} \mathrm{~cm}^{-3}\right)$ relative to HII-region models $\left(10^{2} \mathrm{~cm}^{-3}\right)$ makes He I $\lambda 7065 / \mathrm{He}$ I $\lambda 6678$ rise because of collisional enhancement when the AGN contribution rises, while the inclusion of microturbulence in the AGN models (Section 2.3) reduces the 13889 -line optical depth and hence the effects of fluorescence (Benjamin et al. 2002), causing He I $\lambda 3889 / \mathrm{He}$ I $\lambda 6678$ to also rise (Figs 9d and 11d).

Shock component. The series of red symbols of different darkness in Figs 8-11 show the effect of adding a radiative-shock component contributing 90 per cent of the total He II $\lambda 1640$ emission, using the prescription of Section 2.4. The symbol shape corresponds to the metallicity of the associated benchmark model (upside-down triangle: $Z=0.0005$; circle: $Z=0.002$; square: $Z=0.008$ ) and the darkness to the shock velocity (from $10^{2} \mathrm{~km} \mathrm{~s}^{-1}$ : light; to $10^{3} \mathrm{~km} \mathrm{~s}^{-1}$ : dark). The signatures of a shock component are very similar to those identified above for an AGN component, in particular very strong ratios of He II-to-other lines, the fraction of, for example, total $\mathrm{H} \beta$ luminosity shocks account for being typically less than 15 per cent in Figs 8-11. This is because collisional ionization in the high-temperature $\left(T_{\mathrm{e}}>10^{6} \mathrm{~K}\right)$ radiative zone of a shock produces $\mathrm{He}^{2+}$ along with other highly-ionized species (e.g., $\mathrm{C}^{4+}$ to $\mathrm{C}^{6+}, \mathrm{N}^{4+}$ to $\mathrm{N}^{7+}, \mathrm{O}^{4+}$ to $\mathrm{O}^{8+}$ ), whose recombination generates strong He II emission and extreme ultraviolet and soft $\mathrm{X}$-ray emission capable of producing lower-ionization species upstream and downstream of the shock (see figs. 8-11 of Allen et al. 2008). Hence, the main effect of adding a contribution by shock-ionized gas is to raise the He II $\lambda 1640$ equivalent width (Figs $8 \mathrm{~b}$ and 10b) and all ratios of He II-to-other lines, such as He II $\lambda 1640 / O$ III] (Figs $8 \mathrm{~h}$ and 10h) and He II $\lambda 4686 / \mathrm{H} \beta$ (Figs 9e and 11e), and the inverse of C III]/He II $\lambda 1640$ (Figs 8b and 10b) and C IV/He II $\lambda 1640$ (Figs 8c and 10c). The intensity and hardness of the ionizing radiation increases with shock velocity. We have checked that adopting different pre-shock densities (in the range $1 \leq n_{\mathrm{H}} \leq 10^{4} \mathrm{~cm}^{-2}$; see Section 2.4) and transverse magnetic fields $\left(10^{-4} \leq B \leq 10 \mu \mathrm{G}\right)$ has a negligible effect on the results of Figs 8-11, except for the low-ionization [N II] and [O II] lines (Figs 9a,b and 11a,b), whose fluxes tend to decrease when $n_{\mathrm{H}}$ rises and $B$ drops.

\section{CONSTRAINTS ON THE PRODUCTION AND ESCAPE OF IONIZING RADIATION}

In the previous section, we described the ultraviolet and optical emission-line signatures of a wide range of ISM, stellarpopulation, AGN and radiative-shock parameters in metal-poor 

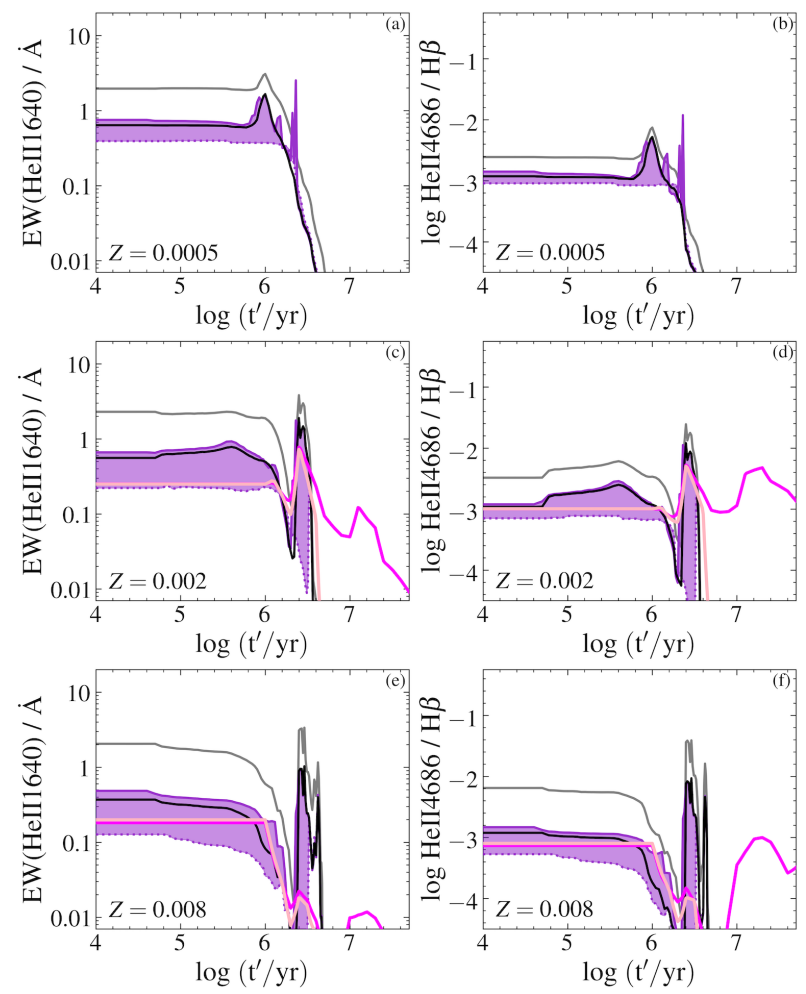

Figure 12. Evolution of the He II $\lambda 1640$ equivalent width (left) and He II $\lambda 4686 / \mathrm{H} \beta$ ratio (right) in models of ionization-bounded HII regions powered by different types of SSPs. The black curves show the C\&B-based benchmark models with $\log \langle U\rangle=-2$ of Section 4 , for $Z=0.0005$ (top), 0.002 (middle) and 0.008 (bottom). The light-purple and magenta curves show the corresponding models powered by BPASS single- and binary-star SSPs, respectively (BPASS models are not available for $Z=0.0005$ ). The dotted and solid dark-purple lines show SSP models with the same parameters as black curves, but for IMF upper mass cut-offs $m_{\mathrm{up}}=100$ and $600 \mathrm{M}_{\odot}$, respectively (the area between these two models has been shaded in purple, for clarity). The grey curves show the same models as the black curves, but for $\log \langle U\rangle=-1$.

star-forming galaxies. We now interpret these results to investigate emission-line diagnostics of the production and escape of ionizing radiation in such galaxies. Specifically, we wish to assess the hints provided by the reference observational sample of Section 3 on the sources dominating the production of ionizing photons (Section 5.1) and on LyC-photon leakage (Section 5.2) in these galaxies. We mention along the way how our findings compare to those of previous studies relying on investigations of often fewer emission lines with different models.

\subsection{Diagnostics of ionizing sources}

Among the most challenging lines to reproduce in the spectra of metal-poor star-forming galaxies are the He II recombination lines, whose strength can be much stronger than predicted by standard models (Section 1). The signatures of the wide collection of models considered in Section 4 in spectral diagnostic diagrams involving He II lines therefore provide potentially useful hints on the sources powering the ionizing radiation in such galaxies. For example, we saw that, in ionization-bounded models, the
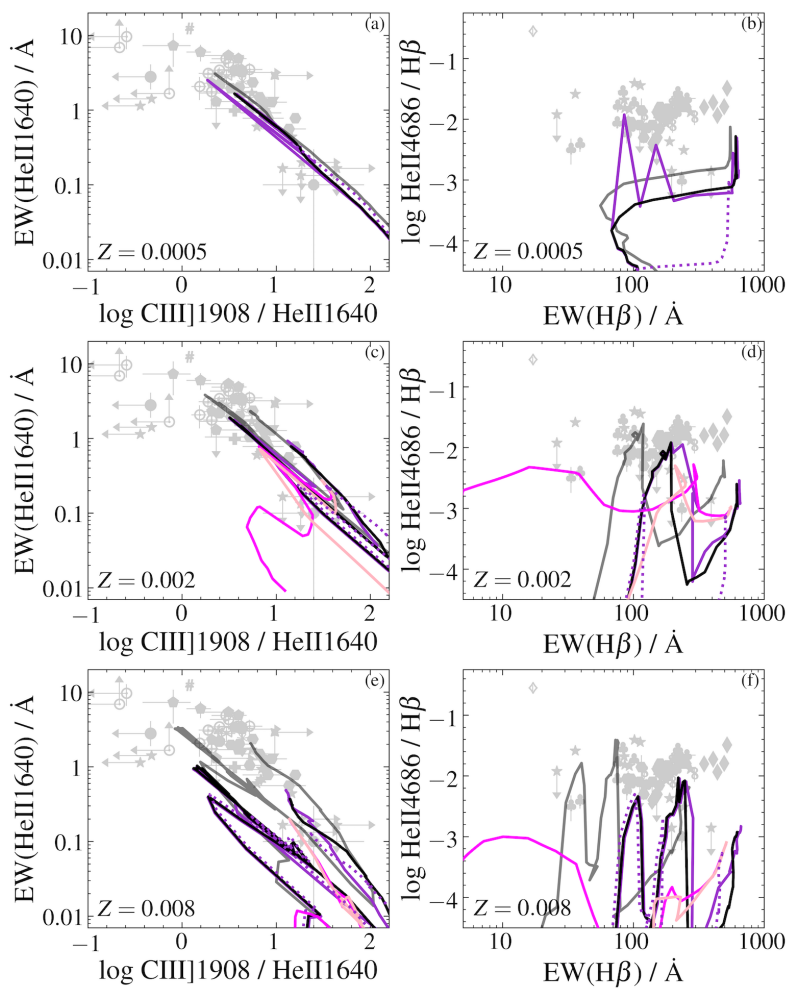

Figure 13. EW(He II $\lambda 1640$ ) plotted against C III]/He II $\lambda 1640$ (left; as in Fig. 6b) and $\mathrm{He} \mathrm{II} \lambda 4686 / \mathrm{H} \beta$ plotted against $\mathrm{EW}(\mathrm{H} \beta)$ (right; as in Fig. 7e). The observations (greyed for clarity) are the same as in Figs. $6 \mathrm{~b}$ and $7 \mathrm{e}$, while the models are the same as in Fig. 12 (without the purple shading between models for $m_{\mathrm{up}}=100$ and $600 \mathrm{M}_{\odot}$ ).

equivalent widths of He II $\lambda 1640$ and $\mathrm{H} \beta$ and the $\mathrm{He}$ II $\lambda 4686 / \mathrm{H} \beta$ ratio depend only moderately on ISM parameters other than $\langle U\rangle$, making these observables selectively sensitive to the source of ionizing radiation. Instead, the ratios of He II-to-metallic lines are also strongly affected by metallicity, the $\mathrm{C} / \mathrm{O}$ ratio (in the case of $\mathrm{C}$ III]/He II $\lambda 1640$ and C IV/He II $\lambda 1640$ ) and interstellar-line absorption (in the case of C IV/He II 21640 ). With this in mind, we investigate below the extent to which stellar populations, AGN and radiative shocks can account for the emission-line signatures of metal-poor, star-forming galaxies. We also discuss X-ray binaries as potential sources of ionizing radiation in these galaxies.

\subsubsection{Stellar populations}

If stars are the main source of ionizing radiation in the galaxies of the reference sample of Section 3, the equivalent widths of $\mathrm{He}$ II $\lambda 1640$ and $\mathrm{H} \beta$ and the $\mathrm{He}$ II $\lambda 4686 / \mathrm{H} \beta$ ratio will depend sensitively on the upper mass cut-off of the IMF, $m_{\text {up }}$, the stellar population age, $t$, the stellar population model itself and $\langle U\rangle$ (Figs 8a, 9e, 10a and 11e). To further characterise this dependence, we show in Fig. 12 the evolution of $\mathrm{EW}(\mathrm{He}$ II $\lambda 1640)$ and He II $\lambda 4686 / \mathrm{H} \beta$ for ionization-bounded HII regions powered by different types of SSPs, while Fig. 13 shows these models in the same panels as in Figs $6 \mathrm{~b}$ and $7 \mathrm{e}$ defined by $\mathrm{EW}(\mathrm{He}$ II $\lambda 1640), \mathrm{C}$ III] $\lambda 1908 / \mathrm{He}$ II $\lambda 1640$, He II $\lambda 4686 / \mathrm{H} \beta$ and $\operatorname{EW}(\mathrm{H} \beta)$. The black curves in Figs 12 and 13 show SSP 

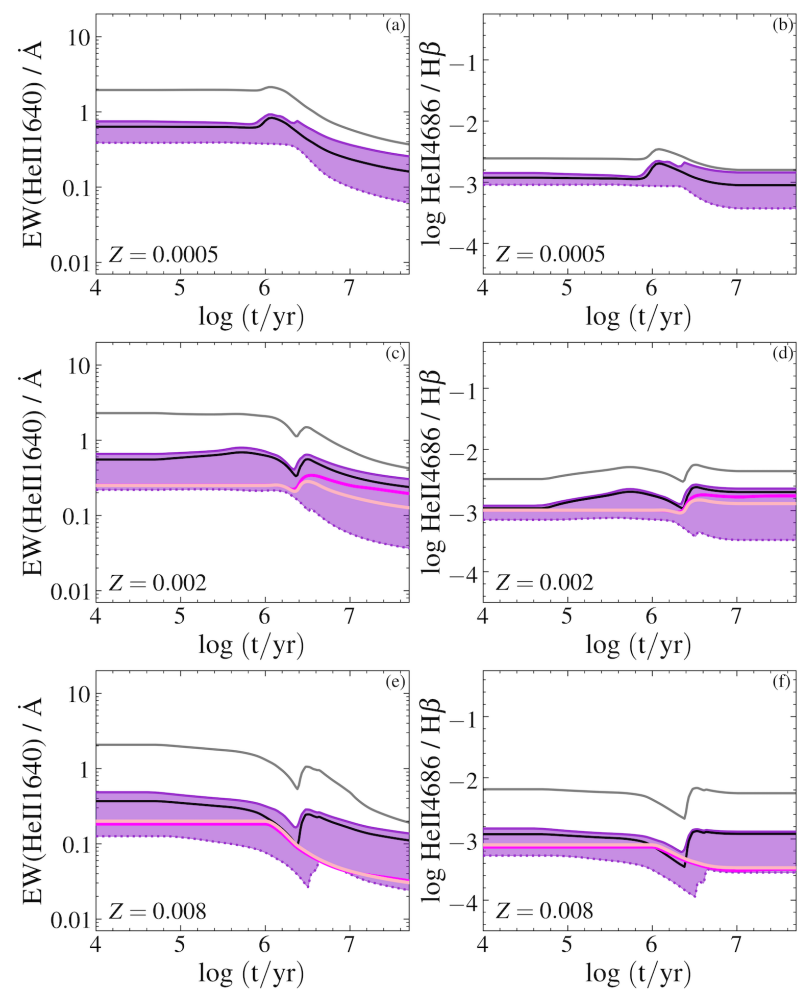

Figure 14. Same as Fig. 12, but for models with constant star formation rate.

models with the same parameters as the benchmark models with $\log \langle U\rangle=-2$ of Section 4, for $Z=0.0005$ (top panels), 0.002 (middle panels) and 0.008 (bottom panels). In Fig. 12, these models show how, as $Z$ increases, the drop in effective temperature of massive stars on the early main sequence (for $t^{\prime} \ll 1 \mathrm{Myr}$ ), the rise in mass-loss rate (a $300 \mathrm{M}_{\odot}$ star leaving the main sequence, around $t^{\prime} \approx 2 \mathrm{Myr}$, has lost 10,25 and 70 per cent of its mass for $Z=0.0005,0.002$ and 0.008 , respectively) and the development of the WR phase (around $t^{\prime} \approx 3 \mathrm{Myr}$ ) shape the evolution of $\mathrm{EW}(\mathrm{He}$ II $\lambda 1640$ ) and He II $\lambda 4686 / \mathrm{H} \beta$ (see also Section 4).

In Figs $13 \mathrm{a}, 13 \mathrm{c}$ and $13 \mathrm{e}$, the above models reach a region populated by galaxies with more extreme $\mathrm{EW}(\mathrm{He}$ II $\lambda 1640)$ and C III]/He II $\lambda 1640$ than could be attained by models with constant star formation rate in Fig. 8b. This is because, as Fig. 14 shows, continuous star formation smoothes out the evolution of the spectral features in Fig. 12. However, while the WR phase of the model with $Z=0.002$ hardly reaches the high observed $\mathrm{He} \mathrm{II} \lambda 4686 / \mathrm{H} \beta \approx 0.01$ around $\mathrm{EW}(\mathrm{H} \beta) \approx 200 \AA$ (Fig. 13d), none of the reference SSP models can account for the extreme He II $\lambda 4686 / \mathrm{H} \beta \approx 0.02$ of galaxies with $\mathrm{EW}(\mathrm{H} \beta) \approx 500 \AA$ in the Izotov et al. (2017) sample (Figs 13b, 13d and 13f). Increasing the ionization parameter from $\log \langle U\rangle=-2$ to -1 (grey curves) significantly boosts EW(He II $\lambda 1640$ ) and He II $\lambda 4686 / \mathrm{H} \beta$ in Fig. 12 (see Section 4), but this improves only moderately the discrepancy between observed and predicted $\mathrm{He}$ II $\lambda 4686 / \mathrm{H} \beta$ for galaxies with $\mathrm{EW}(\mathrm{H} \beta) \approx 500 \AA ̊$ in Fig. 13.

The light-purple and magenta curves in Figs 12-14 show the predictions of the BPASS single- and binary-star models, respectively, for SSPs with the same parameters as the C\&B ref- erence models, for $Z=0.002$ and 0.008 (BPASS models are not available for $Z=0.0005$ ). These models start at $t^{\prime}=1 \mathrm{Myr}$, hence the flat evolution of $\mathrm{EW}(\mathrm{He}$ II $\lambda 1640)$ and $\mathrm{He}$ II $\lambda 4686 / \mathrm{H} \beta$ at younger ages in Fig. 13. From 1 to $3 \mathrm{Myr}$, both BPASS models show qualitatively the same evolution as the C\&B models, albeit with a weaker WR phase implying smaller EW(He II $\lambda 1640$ ) and $\mathrm{He} \mathrm{II} \lambda 4686 / \mathrm{H} \beta$. Then, as the He II emission dies off in the single-star BPASS and C\&B models, the production of hard ionizing radiation is maintained through binary mass transfer in the binary-star BPASS model. The effect is particularly striking in the generation of strong He II $\lambda 4686 / \mathrm{H} \beta$ at small $\mathrm{EW}(\mathrm{H} \beta)$ in Figs $13 \mathrm{~d}$ and 13f. However, this has no effect on the discrepancy between models and observations, which appears to be even more severe at larger $\operatorname{EW}(\mathrm{H} \beta)$ for the BPASS than for the C\&B models. We note in passing that Eldridge et al. (2017) used observations of $[\mathrm{O} \mathrm{III}] / \mathrm{H} \beta$ versus $\mathrm{EW}(\mathrm{H} \beta)$ (Fig. 7c above) to highlight the better performance of binary- versus single-stellar population models. As the brown (age) and magenta (BPASS) segments in Figs 9c and $11 \mathrm{c}$ suggest, star-forming galaxies with low $\mathrm{EW}(\mathrm{H} \beta)$ and high $[\mathrm{O} \mathrm{III}] / \mathrm{H} \beta$ can be reached by both types of models for continuous star formation at ages $t \gg 10 \mathrm{Myr}^{5}$ In fact, we have checked for example that the model with $\log \langle U\rangle=-2$ and $Z=0.002$ in these figures reaches $\mathrm{EW}(\mathrm{H} \beta) \approx 50 \AA$ ( $40 \AA$ ) after $1 \mathrm{Gyr}(2 \mathrm{Gyr})$ of constant star formation, at nearly constant $[\mathrm{O}$ III $] / \mathrm{H} \beta$.

The other parameter strongly affecting the ionizing radiation from young stellar populations is the upper mass cut-off of the IMF. The dotted and solid dark-purple lines in Figs 12-14 show SSP models with the same parameters as the C\&B reference models, but for $m_{\text {up }}=100$ and $600 \mathrm{M}_{\odot}$, respectively. In Figs 12 and 14 , the area between these two models has been shaded in purple, for clarity. While raising $m_{\text {up }}$ hardens the ionizing radiation, the effect is modest from $m_{\text {up }}=300$ and $600 \mathrm{M}_{\odot}$ (Section 4). Fine-tuning the upper IMF therefore does not look promising to improve significantly the agreement between models and observations of $\mathrm{EW}(\mathrm{He}$ II $\lambda 1640$ ), C III]/He II $\lambda 1640, \mathrm{He}$ II $\lambda 4686 / \mathrm{H} \beta$ and $\mathrm{EW}(\mathrm{H} \beta)$ in Fig. 13. This is consistent with the conclusions reached by Stanway \& Eldridge (2019) based simply on the ratio of He II-to-Hi ionizing photons.

We note that models with LyC-photon leakage $\left(f_{\text {esc }}>0\right)$ can reach larger ratios of He II-to-low ionization lines, such as He II $\lambda 1640 / \mathrm{CIII}$ (inverse abscissa of Figs $8 \mathrm{~d}$ and 10d), He II $\lambda 1640 /(\mathrm{C}$ IV $+\mathrm{C}$ III]) (inverse abscissa of Figs 8e and 10e), He II $\lambda 1640 / \mathrm{O} \mathrm{III]}$ (Figs $8 \mathrm{~h}$ and 10h) and He II $\lambda 4686 / \mathrm{H} \beta$ (Figs 9e and $11 \mathrm{e})$. However, such models fail to account simultaneously for the large equivalent widths of low-ionization lines observed in many galaxies (see, e.g., Figs $8 \mathrm{~h}$ and $10 \mathrm{~h}$ for $\mathrm{C} \mathrm{III}]$, and Figs $9 \mathrm{e}$ and $11 \mathrm{e}$ for $\mathrm{H} \beta$ ).

\subsubsection{AGN and radiative shocks}

Figs 8-11 show how introducing either an AGN or radiativeshock component allows models to reproduce observations of

\footnotetext{
${ }^{5}$ In the version of Fig. 7c published by Eldridge et al. (2017, fig. 38 in their paper), only SSP models were presented, and the $\mathrm{H} \beta$ equivalent widths from Schenker et al. (2013) were inadvertently corrected twice for redshift (J. J. Eldridge, private communication).
} 


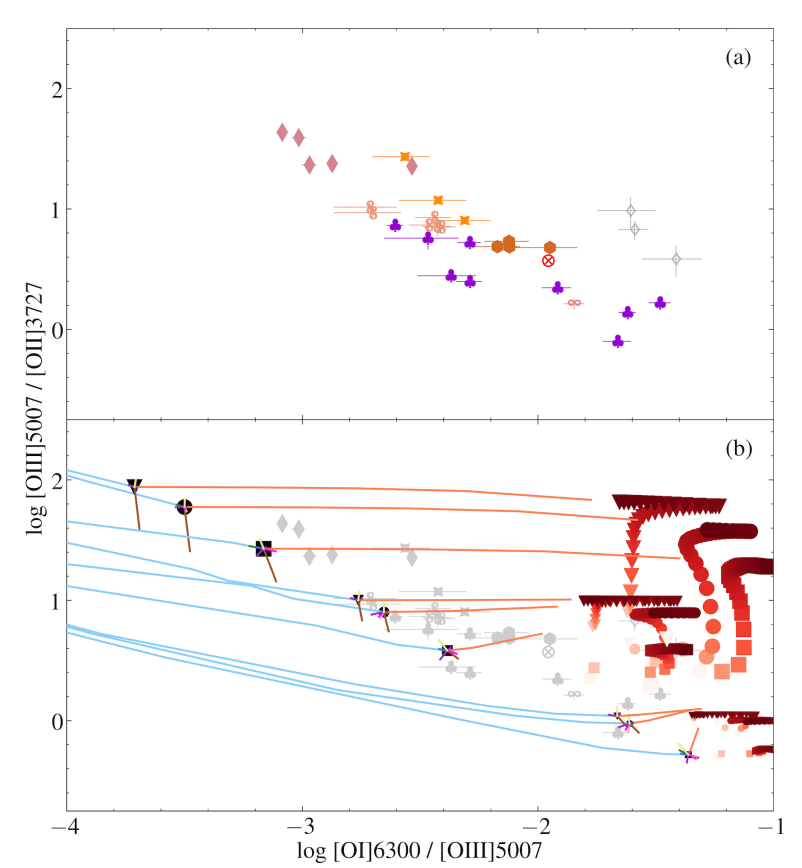

Figure 15. [O III $] /[\mathrm{O}$ II] plotted against [O I $] /[\mathrm{O}$ III] for: (a) the galaxies in the reference sample of Section 3 (available in practice only for the subsample of LyC leakers and two AGN); and (b) the complete set of models from Figs 8-11, along with observations from panel (a) greyed for clarity.

galaxies with high He II emission in nearly all ultraviolet and optical line-ratio diagrams. This is not surprising, given the strong He II emission produced by AGN and radiative shocks (Section 4), which has long made them good candidate sources of hard ionizing radiation in metal-poor star-forming galaxies (Section 1). The novelty of Figs $8-11$ is to illustrate at once, and with a self-consistent modelling of interstellar abundances, the influence of these components on a wide range of ultraviolet and optical emission lines. Also, having assembled a substantial observational reference sample (Section 3) allows us to highlight general trends and derive more robust conclusions than based on individual objects.

It is not obvious from Figs $8-11$ which of an AGN or radiative-shock component can best account for the properties of metal-poor star-forming galaxies with strong He II emission. Izotov et al. (2012) find that the production of high-ionization [Ne V] $\lambda 3426$ emission $\left(E_{\text {ion }}>97.2 \mathrm{eV}\right)$ in the spectra of 8 blue compact dwarf galaxies with $12+\log (\mathrm{O} / \mathrm{H})=7.3-7.7$ and strong He II emission (He II $\lambda 4686 / \mathrm{H} \beta \gtrsim 0.01$ ) requires a contribution of about 10 per cent of the total ionizing radiation by AGN or radiative shocks. While these authors favour supernova-driven radiative shocks with velocities around $300-500 \mathrm{~km} \mathrm{~s}^{-1}$ as the source of this emission, they cannot rule out an AGN origin. Stasińska et al. (2015) also note that shocks can naturally account for the high [O I] $\lambda 6300 /[\mathrm{O}$ III] ratios observed in the spectra of blue compact dwarf galaxies with high $[\mathrm{O}$ III $] /[\mathrm{O}$ II] ratios, as density-bounded models producing high $[\mathrm{O} \mathrm{III}] /[\mathrm{O} \mathrm{II}]$ would imply low [O I]/[O III] (see also Section 2.2).

To further investigate this issue, in Fig. 15, we plot
$[\mathrm{O}$ III $] /[\mathrm{O}$ II $]$ versus $[\mathrm{O} \mathrm{I}] /[\mathrm{O} \mathrm{III}]$ for the complete set of models from Figs 8-11, along with observations from the sample of Section 3 - available in practice only for the subsample of LyC leakers (Leitet et al. 2011; Jaskot \& Oey 2013; Izotov et al. 2016a,b, 2017, 2018a,b; Chisholm et al. 2017), a few Wolf-Rayet galaxies (López-Sánchez \& Esteban 2008) and three AGN (Dors et al. 2014). As in other line-ratio diagrams, contributions by AGN and radiative shocks to the ionizing radiation of model galaxies have roughly similar signatures in Fig. 15, increasing $[\mathrm{O} \mathrm{I}] /[\mathrm{O} \mathrm{III}]$ typically far more than $[\mathrm{O} \mathrm{III}] /[\mathrm{O} \mathrm{II}]$ (except for very low ionization parameter). Hence, these line ratios cannot either help discriminate at first glance between AGN and shock ionization in a galaxy. A more striking feature of Fig. 15 is that nearly all observations of (confirmed and candidate) LyC leakers exhibit higher [O I] /[O III] than the benchmark ionization-bounded models in the full explored ranges of $-3 \leq \log \langle U\rangle \leq-1$ and $0.0005 \leq Z \leq 0.008$ at fixed [O III $] /[\mathrm{O}$ II] . Tuning the stellar population parameters, including the star formation history, can bring the models only slightly closer to the data. In densitybounded models, the ratio of low- to high-ionization lines further drops (Section 2.2), worsening the agreement between models and observations (light-blue segments in Fig. 15). The only way to account for the observed properties of LyC leakers in Fig. 15 is to invoke a significant contribution by an AGN or radiative shocks (or X-ray binaries, but see Section 5.1.3 below) to the ionizing radiation. This is because the hard penetrating X-ray and extreme-ultraviolet radiation from such sources produces higher electronic temperatures than stellar radiation in the outskirts of HII regions, thereby enhancing $\mathrm{O}$ I collisional excitation (we note that, in young shocks which have not yet developed a cool tail, $[\mathrm{OI}] /[\mathrm{O}$ III $]$ can be significantly reduced and $\mathrm{He} \mathrm{II} / \mathrm{H} \beta$ slighly enhanced relative to the models shown in Fig. 15; see Alarie \& Morisset 2019). This can arise in the context of both densitybounded and ionization-bounded (in the picket-fence leakage scenario; see Section 2.2) models. This conclusion is consistent with that drawn by Stasińska et al. (2015) from the analysis of a sample of blue compact dwarf galaxies with very high excitation.

Stasińska et al. (2015) also pointed out the interest of the [Ar III] $\lambda 7135$ and [Ar IV] $\lambda 4740$ (hereafter simply [Ar III] and [ArIV]) lines to probe ionizing-photon energies greater than the ionization potential of $\mathrm{Ar}^{2+}(40.7 \mathrm{eV})$, which lies between the ionization potentials of $\mathrm{O}^{+}(35.1 \mathrm{eV})$ and $\mathrm{He}^{+}(54.4 \mathrm{eV})$. In Fig. 16, we plot [O III]/[O II] against [Ar IV]/[Ar III] for the same models and observations as in Fig. 15; in practice, data are available only for a few candidate LyC leakers (Jaskot \& Oey 2013; Izotov et al. 2017) and an AGN (Dors et al. 2014). The benchmark ionization-bounded models appear to overlap with the data in this diagram, as do density-bounded models, eventually combined with an AGN or radiative-shock component. Along with this smaller dispersion of models relative to Fig. 15, is worth noting that, in Fig. 16, only models with a radiative-shock component can reach $[\mathrm{Ar} I V] /[$ Ar III $] \sim 1$ around $[\mathrm{O} \mathrm{III}] /[\mathrm{O}$ II $] \sim 10$, where some extreme-excitation galaxies can be found in the Stasińska et al. (2015) sample (see their fig. 6).

Radiative shocks from expanding Hir regions and supernova blast waves are an appealing natural hypothesis for the origin of hard ionizing radiation in actively star-forming, metalpoor galaxies (e.g., Thuan \& Izotov 2005; Stasińska et al. 2015). Fig. 15 supports the idea that shocks may be intimately related to the leakage of ionizing photons from such galaxies. Interestingly, 


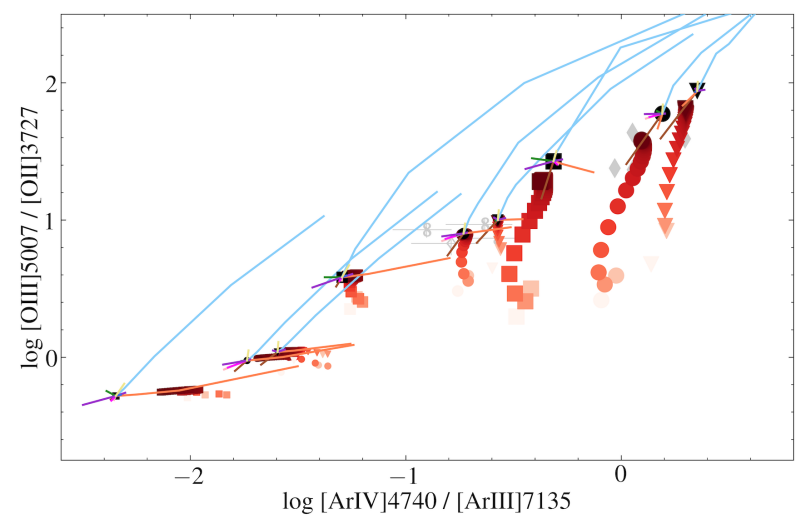

Figure 16. Same as Fig. 15b, but for $[\mathrm{O} \mathrm{III}] /[\mathrm{O} I \mathrm{II}]$ plotted against [Ar IV]/[Ar III]

the presence of shocks will increase primarily the luminosities of $\mathrm{He}$ II and very-high-ionization lines, such as [Ne V] $\lambda 3426$ (but not so much $\mathrm{N} \mathrm{V} \lambda 1240$, as $\mathrm{N}^{4+}$ is converted into $\mathrm{N}^{5+}$; see Section 4), while the luminosities of lower-ionization lines (including $\mathrm{H} \beta$ ) remain largely controlled by stellar radiation. In fact, Izotov et al. (2012) find no significant correlation between [Ne V] and $\mathrm{H} \beta$ emission in the 8 galaxies of their sample. In this context, the absence of correlation between $\mathrm{He}$ II and $\mathrm{H} \beta$ emission in the sample of extremely metal-poor galaxies studied by Senchyna \& Stark (2019, see also Senchyna et al. 2019) could be consistent with a radiative-shock origin of the He II emission.

AGN and radiative-shock components are sometimes discarded as sources of hard ionizing radiation on the basis of spectral fits. For example, Berg et al. (2018) conclude that an AGN or radiative-shock component is unlikely to account for the strong He II $\lambda 1640$ emission in the extreme star-forming galaxy SL2SJ021737-051329, as this would make C III]/O III] too small and C IV/C III] too large, based on AGN models by Groves et al. (2004) and shock models by Allen et al. (2008, see Table 1). While the HII-region, AGN and shock models used by Berg et al. (2018) were computed using different ISM prescriptions, in the framework of our models, as can be guessed from Figs 8-11, a combination of $\log \langle U\rangle \lesssim-2, \mathrm{C} / \mathrm{O} \gtrsim 0.17, \xi_{\mathrm{d}} \approx 0.1$ and an AGN (or radiative-shock) contribution of $\sim 8$ per cent of the total $\mathrm{H} \beta$ emission turns out to accommodate the observed ultraviolet and optical nebular spectrum of this galaxy (see Figs 6 and 7 to locate the galaxy in all panels, the oxygen abundance corresponding to a metallicity between $Z=0.0005$ and 0.002). ${ }^{6}$ Hence, in some cases, the assessment of the potential presence of an AGN or radiative-shock component in a galaxy may depend on the adopted model prescription, highlighting once more the importance of a physically-consistent modelling of nebular emission from different sources (Section 2). We also recall that the AGN models presented in this paper were computed for a typical ionizing-spectrum slope $\alpha=-1.7$ (Section 2.3), and that $\alpha$ vari-

\footnotetext{
6 A pure SSP of age $t^{\prime} \approx 2.5 \mathrm{Myr}$ with the same $\langle U\rangle$, C/O and $\xi_{\mathrm{d}}$ as this composite model can also approximate closely all observations of SL2SJ021737-051329 in Figs 8-11, except for the $\mathrm{H} \beta$ equivalent width $[\mathrm{EW}(\mathrm{H} \beta) \approx 200 \AA$ instead of the observed $517 \AA]$. We consider this model less likely because of the very specific age required.
}

ations could imply significant dispersion in predicted ultraviolet and optical line ratios (e.g., Feltre et al. 2016).

It is worth noting that while a radiative-shock or AGN component can readily accommodate the emission-line properties of many observed galaxies with strong He II $\lambda 1640$ emission in Figs 8-11, including those with weak C IV/C III] (see above; there is also the possibility for C IV emission to be reduced via interstellar absorption; Section 4), some outlier galaxies exhibit properties not sampled by the limited set of models presented here. We have checked that some models can account for the properties of such galaxies. For example, we find that galaxies with $\mathrm{EW}(\mathrm{He}$ II $\lambda 1640) \gtrsim 2 \AA$ and $\mathrm{C}$ III] $/ \mathrm{He}$ II $\lambda 1640 \gtrsim 4$ (Figs $8 \mathrm{~b}$ and $10 \mathrm{~b}$ ) can be reached by models with $\mathrm{C} / \mathrm{O} \gtrsim 0.17$ and a radiative-shock or AGN component. In Figs 8c and 10c, the observed $\mathrm{EW}(\mathrm{C}$ IV $) \gtrsim 20 \AA$ and $\mathrm{CIV} / \mathrm{He}$ II $\lambda 1640 \approx 10$ of the lensed double-super star cluster ID14 (Vanzella et al. 2017, whose properties approach those of the Hir galaxy of Fosbury et al. 2003) can be accommodated by young ( $t \sim 1 \mathrm{Myr})$, high-ionization $(\log \langle U\rangle \sim-1)$ models with $\mathrm{C} / \mathrm{O} \gtrsim 0.17$ and $\xi_{\mathrm{d}} \lesssim 0.3$, also compatible with the other emission-line properties of this object. Very young models with $\mathrm{C} / \mathrm{O} \gtrsim 0.17$ can also reach galaxies with high $\mathrm{EW}(\mathrm{C} \mathrm{III}])$ at small $\mathrm{C}$ IV/C III] in Figs $8 \mathrm{~g}$ and $10 \mathrm{~g}$, while the Laporte et al. (2017) galaxy, with low C III]/He II $\lambda 1640$ and high $\mathrm{N}$ V/He II $\lambda 1640$, could well be a LyC-photon leaker powered by radiative shocks or an AGN (Figs $8 \mathrm{~g}$ and $10 \mathrm{~g}$ ). The above rough exploration of the parameter space will need to be refined by more robust spectral fits of each galaxy in the sample, using tools such as BEAGLE (Chevallard \& Charlot 2016), extended to incorporate AGN and radiative-shock prescriptions.

\subsubsection{X-ray binaries}

$\mathrm{X}$-ray binaries, in which a compact object (neutron star or stellarmass black hole) accretes material from a massive O/B companion, have been proposed as natural sources of hard ionizing photons in metal-poor star-forming galaxies (e.g., Garnett et al. 1991). An argument supporting this hypothesis is the observed increase in hard X-ray luminosity with decreasing oxygen abundance (at a fixed star formation rate) in nearby metal-poor star-forming galaxies (Brorby et al. 2016, and references therein), which goes in the same sense as the increase in $\mathrm{EW}$ (He II $\lambda 1640$ ) (Fig. 6a) and He II $\lambda 4686 / \mathrm{H} \beta$ (Fig. 7f). Also, the non-correlation of the equivalent width of $\mathrm{He}$ II $\lambda 4686$ with that of $\mathrm{H} \beta$ and other emission lines in the sample of extremely metal-poor galaxies studied by Senchyna \& Stark (2019) suggests that $\mathrm{He}^{+}$-ionizing photons are produced by sources with timescales greater than massive O/B stars, such as stripped stars produced by close binary evolution and X-ray binaries. The accretion physics of X-ray binaries presents similarities to that of AGN (see the review by Gilfanov \& Merloni 2014), which are in fact often considered as scaled-up versions of X-ray binaries (e.g., McHardy et al. 2006). Hence, X-ray binaries are expected to produce ionizing spectra similar to those of AGN (see also fig. C5 of Stasińska et al. 2015), implying effects on emission-line ratios and equivalent widths similar to those found for an AGN component in Figs 8-11.

Recently, Schaerer et al. (2019) computed the time evolu- 
tion of He II $\lambda 4686 / \mathrm{H} \beta$ and $\mathrm{EW}(\mathrm{H} \beta)$ for SSPs including X-ray binaries at different metallicities, by combining the Fragos et al. (2013, see also Madau \& Fragos 2017) stellar population synthesis models of X-ray binaries with BPASS v2.1, and adopting an approximate conversion between X-ray luminosity and rate of $\mathrm{He}^{+}$-ionizing photons. This model reproduces roughly the trend of increasing He II $\lambda 4686 / \mathrm{H} \beta$ with decreasing $12+\log (\mathrm{O} / \mathrm{H})$ in nearby metal-poor star-forming galaxies (fig. 1 of Schaerer et al. 2019). However, it fails to account for the high $\mathrm{He}$ II $\lambda 4686 / \mathrm{H} \beta$ ratios of galaxies with large $\mathrm{EW}(\mathrm{H} \beta)$, just as the other stellar population synthesis models considered in Fig. 13 above (see fig. 3 of Schaerer et al. 2019). This is consistent with the finding that X-ray binaries have spectra too soft to account for the very hard ionizing radiation of some metal-poor star-forming galaxies (e.g., Thuan \& Izotov 2005; Izotov et al. 2012; Stasińska et al. 2015), that they appear on too-long timescales to account for the emission-line properties of Green-Pea galaxies (Jaskot \& Oey 2013) and with the stringent observational upper limit from Chandra on the presence of X-ray binaries in the most extreme He II-emitter observed by Senchyna et al. (2017). We conclude that, while X-ray binaries may provide a natural source of hard ionizing photons in metal-poor star-forming galaxies, they cannot account for the entire emission observed in the most extreme, highest-ionization cases.

\subsection{Diagnostics of LyC-photon leakage}

We now focus on the models of density-bounded Hir regions in Figs 8-11, to assess whether the emission-line properties of metal-poor star-forming galaxies can provide useful constraints on the fraction of escaping LyC photons, $f_{\text {esc }}$. As seen in Section 2.2 (and references therein), increasing $f_{\text {esc }}$ removes the outer low-ionization zones of Hı regions, making ratios of highto low-ionization lines (e.g. $[\mathrm{OIII}] /[\mathrm{OII}]$ ) rise and the equivalent widths of lines with low ionization potential (e.g. C III]) drop. The interpretation of these signatures in galaxy spectra is unfortunately complicated by the competing effects of other galaxy physical parameters, in particular the nature of the ionizing source, the ionization parameter, $\langle U\rangle$, metallicity, $Z$, and to a lesser extent the gas density, $n_{\mathrm{H}}$, dust-to-metal mass ratio, $\xi_{\mathrm{d}}$, and $\mathrm{C} / \mathrm{O}$ ratio (Figs 8-11; see also Jaskot \& Oey 2013; Nakajima \& Ouchi 2014; Stasińska et al. 2015; Jaskot \& Ravindranath 2016; Izotov et al. 2017). These degeneracies between the spectral signatures of $f_{\text {esc }}$ and other parameters are the reason why LyC leakers appear to overlap with the rest of the population of actively star-forming galaxies in Figs 6 and 7 (see Section 3.4).

Several diagnostics must therefore be combined to potentially discriminate the effects of $f_{\text {esc }}$ from those of other parameters on emission-line ratios. That $[\mathrm{O} \mathrm{III}] /[\mathrm{O}$ II $]$ alone is not a sufficient condition for LyC leakage is also illustrated by the fact that, as seen in Section 2.2 (Fig. 5f), this ratio for a densitybounded galaxy with constant star formation can actually be smaller than that of an ionization-bounded one for large $f_{\text {esc }}$ and $\langle U\rangle$ (see age effect on model with $\log \langle U\rangle=-1$ in Fig. 11b). Jaskot \& Ravindranath (2016) suggest that, for example, high $[\mathrm{O} \mathrm{III}] /[\mathrm{O} \mathrm{II}](\gtrsim 10)$ and low $\mathrm{EW}(\mathrm{C} \mathrm{III}])(\lesssim 4 \AA)$ will tend to select density-bounded galaxies, although they do acknowledge that the scaling of $f_{\text {esc }}$ with $\mathrm{EW}(\mathrm{C}$ III] $)$ will depend sensitively on metal abundances and stellar population age, as Figs $8 \mathrm{~g}$ and $9 \mathrm{~b}$ show. We note in this context that the He I-based $f_{\text {esc }}$ diagnostic proposed by Izotov et al. (2017, see Figs 9d and 11d) requires independent constraints on $n_{\mathrm{H}},\langle U\rangle$ and $Z$. In practice, Figs 8-11 reveal that few observations fall in regions of diagrams populated purely by density-bounded models (in Figs $13 \mathrm{~d}$ and $13 \mathrm{f}$, galaxies with low $\mathrm{H} \beta$ equivalent width and high $[\mathrm{O}$ III] $/ \mathrm{H} \beta$ can be accounted for by ionization-bounded models with ages greater than $10 \mathrm{Myr}$; see Section 5.1.1).

It is also interesting to note that, for the low-mass starforming galaxy $\mathrm{BX} 418$ with low $\mathrm{EW}(\mathrm{H} \beta) \approx 44 \AA$ and high $[\mathrm{O}$ III $] /[\mathrm{O}$ II $]>26$ (using the $1 \sigma$ limit on the [O II] flux), Erb et al. (2010) constrain an age less than $100 \mathrm{Myr}$ from ultraviolet and $\mathrm{H} \alpha$ observations as well as dynamical arguments. This young age, despite the location of $\mathrm{BX} 418$ at low $\mathrm{EW}(\mathrm{H} \beta)$ and high $[\mathrm{O} \mathrm{III}] / \mathrm{H} \beta$ in Figs $9 \mathrm{c}$ and $11 \mathrm{c}$, is suggestive of the fact that the galaxy might be leaking LyC photons, which would be compatible with the other properties of the galaxy in Figs 8$11 .^{7}$ In comparison, the confirmed, per-cent level LyC leakers Haro 11, Tol-0440-381 and Tol-1247-232 (Leitet et al. 2011; Chisholm et al. 2017, see Table 2) also exhibit somewhat low $\mathrm{EW}(\mathrm{H} \beta) \sim 40-100 \AA$ and high $[\mathrm{O} \mathrm{III}] / \mathrm{H} \beta$ in these figures, but with more modest [O III]/[O II] around 1.5-4.0 (Figs $9 \mathrm{~b}$ and $11 \mathrm{~b}$ ), consistent with a picket-fence leakage scenario (Section 2.2; see also Leitet et al. 2011), in addition to density-bounded HII regions.

Hence, assessing whether a galaxy is leaking LyC photons based on the emission-line diagrams in Figs 8-11 is not straightforward at first glance. Several diagnostics must be examined simultaneously to discriminate the signatures of $f_{\text {esc }}$ from those of other physical parameters, which can be best achieved with a full spectral analysis tool incorporating density-bounded models.

\section{CONCLUSIONS}

We have explored the constraints on the production and escape of ionizing photons in young galaxies by investigating the ultraviolet and optical emission-line properties of a broad collection of models relative to the observations of a reference sample of metal-poor star-forming galaxies and $\mathrm{LyC}$ leakers at various redshifts. A main feature of our study is the adoption of models of HII regions, AGN narrow-line regions and radiative shocks computed all using the same physically-consistent description of element abundances and depletion on to dust grains down to metallicities of a few per cent of solar (from Gutkin et al. 2016). We computed ionizing spectra of single- and binary-star populations using the most recent versions of the Bruzual \& Charlot (2003) and Eldridge et al. (2017) stellar population synthesis codes and explored models of ionization-bounded as well as density-bounded (i.e., optically thin to LyC photons) Hiı regions. To compute emission-line spectra of AGN narrow-line regions, we appealed to an updated version of the Feltre et al.

\footnotetext{
7 An ionization-bounded model with $\log \langle U\rangle=-1$ can reach $\mathrm{EW}(\mathrm{H} \beta) \approx 44 \AA$ after about 100 Myr of constant star formation, although the corresponding $\mathrm{He}$ II $\lambda 1640 / \mathrm{O}$ III] is too small relative to the observed one in Fig. 10h (which pertains to the 25-per-cent nebular contribution to the total He II emission of this object; see Erb et al. 2010).)
} 
(2016) models, while for radiative shocks we adopted the recent computations of Alarie \& Morisset (2019).

The observational sample assembled to constrain these models incorporates data from 13 subsamples of metalpoor star-forming galaxies (Table 1), 9 subsamples of confirmed and candidate LyC leakers (Table 2), as well as a few more quiescent star-forming galaxies and AGN at redshifts out to $z=7.1$. The combined sample of closest known analogues to reionization-era galaxies in Tables 1 and 2 allows the simultaneous exploration of diagnostic diagrams involving the $\mathrm{NV} \lambda 1240$, CIV $\lambda \lambda 1548,1551$, He II $\lambda 1640$, O III $] \lambda \lambda 1661,1666, \quad$ [C III $] \lambda 1907+C$ III $] \lambda 1909$, [O II] $\lambda \lambda 3726,3729$, Не I $\lambda 3889$, Не II $\lambda 4686, \mathrm{H} \beta$, [O III] $\lambda 5007$, $\mathrm{H} \alpha$, [N II] $\lambda 6584$, He I $\lambda 6678$ and $\mathrm{He}$ I $\lambda 7065$ emission lines, of which only a few are typically available at once for individual subsamples. This sample shows that, overall, metal-poor starforming galaxies in wide ranges of redshift populate similar regions of the diagrams (but see Senchyna et al. 2019), while LyC leakers tend to overlap with the most extreme star-forming galaxies (Figs 6 and 7).

In agreement with many previous studies, we find that current single- and binary-star population synthesis models do not produce hard-enough ionizing radiation to account for the strong He II emission observed in the most metal-poor star-forming galaxies, even when tuning the IMF. Interestingly, the updated C\&B version of the Bruzual \& Charlot (2003) single-star models used here, which differs from that described by Gutkin et al. (2016, see also Vidal-García et al. 2017) in the inclusion of updated spectra for hot massive stars, produces altogether more He II-ionizing radiation than the binary-star BPASS v2.2.1 models, providing slightly better agreement with the observations (Section 5.1.1 and Figs 12-14). Since a majority of massive stars are expected to undergo binary interactions (e.g., Sana et al. 2012), we consider the He II luminosity predicted by the singlestar C\&B models as a lower limit, which binary-star models (currently under development) will likely exceed. Also, for completeness, since the [O IV] $25.9 \mu \mathrm{m}$ line is often discussed in the same context as the He II line (e.g. Schaerer \& Stasińska 1999), we checked that the [O IV] $25.9 \mu \mathrm{m} /[\mathrm{O}$ III $] \lambda 5007$ ratio in our models behaves similarly to the $\mathrm{He} \mathrm{II} \lambda 4686 / \mathrm{H} \beta$ ratio (this is even more true for the [O IV] $25.9 \mu \mathrm{m} /[\mathrm{O}$ III] $51.8 \mu \mathrm{m}$ ratio, which is less sensitive to electronic temperature).

Introducing hard ionizing radiation from either an AGN or radiative-shock component allows models to overlap with observations of galaxies with high He II emission in nearly all the ultraviolet and optical line-ratio diagrams we investigated. On an object-by-object basis, we find that the conclusion drawn about the potential presence of such ionizing sources using our models can differ from those derived previously using libraries of AGN and radiative-shock models computed with inconsistent descriptions of element abundances. Both AGN and radiativeshock components have very similar signatures in all diagrams, which prevents a simple discrimination between the two at first glance. Similarly, no diagram provides a simple discrimination between LyC-leaking and ionization-bounded galaxies, because of degeneracies in the signatures of $f_{\text {esc }}$ and other galaxy physical parameters. This is the case also in the [O III] $\lambda 5007 /[\mathrm{O}$ II $] \lambda 3727$ versus $[\mathrm{O}$ I] $\lambda 6300 /[\mathrm{O}$ III $] \lambda 5007$ diagram, in which all observations of (confirmed and candidate) LyC leakers exhibit higher
$[\mathrm{OI}] /[\mathrm{O}$ III $]$ than benchmark ionization-bounded models. This is surprising, because density-bounded models produce lower $[\mathrm{O} \mathrm{I}] /[\mathrm{O} \mathrm{III}]$ than ionization-bounded ones at fixed [O III $] /[\mathrm{O} \mathrm{II}]$ (Section 2.2 and Fig. 15; see also Stasińska et al. 2015). The only way to account for the observed properties of LyC leakers in this diagram is to invoke a systematic significant contribution by a source of hard ionizing radiation.

Another potential source of hard ionizing radiation is X-ray binaries, the predicted growing importance of these systems toward low metallicity being supported by the observed increase in hard X-ray luminosity with decreasing oxygen abundance (at fixed star formation rate) in nearby metal-poor star-forming galaxies (Fragos et al. 2013; Brorby et al. 2016). Adopting an approximate conversion of X-ray luminosity into rate of $\mathrm{He}^{+}$ionizing photons allows one to reproduce roughly the observed rise in He II $\lambda 4686 / \mathrm{H} \beta$ ratio with decreasing oxygen abundance in such galaxies (Schaerer et al. 2019). However, like other stellar population synthesis models, this fails to account for the high observed $\mathrm{He}$ II $\lambda 4686 / \mathrm{H} \beta$ ratios of galaxies with large $\mathrm{EW}(\mathrm{H} \beta)$. A source of harder ionizing radiation must be invoked in these extreme objects, such as an AGN or radiative-shock component.

So far, no predictive model has been proposed to link shocks to other galaxy properties and account for, notably, the increase in He II-emission strength with decreasing metallicity. Potential avenues to be explored might be an IMF bias toward massive stars at low metallicities (e.g., Marks et al. 2012) or the higher specific star formation rates of metal-poor dwarf galaxies relative to their more metal-rich, massive counterparts (e.g., Kauffmann et al. 2006; Yates et al. 2012). Both effects would tend to enhance the incidence of radiative shocks from massive stars and supernova blast waves in metal-poor relative to metal-rich galaxies. We also note that gas compression associated with radiative shocks will generate high densities (e.g., Allen et al. 2008). In this context, the high gas densities $\left(n_{\mathrm{H}} \gtrsim 10^{4} \mathrm{~cm}^{-3}\right)$ measured from the [C III] $\lambda 1907+$ C III $] \lambda 1909$ doublet in some distant, lowmetallicity, actively star-forming galaxies (e.g., Maseda et al. 2017; James et al. 2018) could be suggestive of the presence of radiative shocks. The possibility that fast radiative shocks provide the hard radiation necessary to power strong He II emission in metal-poor star-forming galaxies may be tested using high-quality observations of nearby galaxies. In a related paper (Chevallard et al., in preparation), we appeal to spatiallyresolved observations of the extremely metal-poor compact dwarf galaxy SBS0335-052E to quantify the relative contributions from supernova-driven radiative shocks and massive stars to the total He II-ionizing emission from this galaxy.

While the ultraviolet and optical emission-line diagrams of Figs 6 and 7 do not allow simple by-eye diagnostics of the nature of ionizing sources and the escape of $\mathrm{LyC}$ photons in metal-poor star-forming galaxies, differences exist in the spectral signatures of these parameters, which should enable more stringent constraints from simultaneous fits of several lines. This can be best achieved in a Bayesian framework using versatile spectral analysis tools incorporating a physically-consistent description of the sources and transfer of radiation in a galaxy, such as the BEAGLE tool (Chevallard \& Charlot 2016). Although this tool was already shown to reproduce remarkably well the fluxes of 20 ultraviolet and optical (not including He II) emission lines in 10 extreme nearby star-forming regions (Chevallard et al. 2018), 
the current version of the code does not incorporate models for density-bounded HII-regions, narrow-line regions of AGN and radiative shocks. The implementation of these components, in progress, should enable valuable constraints on the production and escape of ionizing radiation from the emission-line spectra of metal-poor star-forming galaxies, and soon of reionization-era galaxies observed by JWST.

\section{ACKNOWLEDGEMENTS}

We are grateful to D. Erb, M. Hirschmann, P. Petitjean, P. Senchyna, D. Stark and A. Wofford for helpful discussions. We also thank M. Mignoli for providing us with line-flux measurement in the average spectrum of type-2 AGN from Mignoli et al. (2019). AF, SC, GB, AF and AVG acknowledge financial support from the European Research Council (ERC) via an Advanced Grant under grant agreement no. 321323-NEOGAL. AF acknowledges support from the ERC via an Advanced Grant under grant agreement no. 339659-MUSICOS. GB acknowledges financial support from DGAPA-UNAM through PAPIIT project IG100319. CM acknowledges financial support through grant CONACyT-CB2015-254132.

\section{REFERENCES}

Alarie A., Morisset C., 2019, arXiv e-prints, p. arXiv:1908.08579 Alexandroff R. M., Heckman T. M., Borthakur S., Overzier R., Leitherer C., 2015, ApJ, 810, 104

Allen M. G., Groves B. A., Dopita M. A., Sutherland R. S., Kewley L. J., 2008, ApJS, 178, 20

Amorín R., et al., 2017, Nature Astronomy, 1, 0052

Baldwin J. A., Phillips M. M., Terlevich R., 1981, PASP, 93, 5

Benjamin R. A., Skillman E. D., Smits D. P., 2002, ApJ, 569, 288

Berg D. A., Skillman E. D., Henry R. B. C., Erb D. K., Carigi L., 2016, ApJ, 827, 126

Berg D. A., Erb D. K., Auger M. W., Pettini M., Brammer G. B., 2018, preprint, (arXiv: 1803.02340)

Berg D. A., Erb D. K., Henry R. B. C., Skillman E. D., McQuinn K. B. W., 2019, ApJ, 874, 93

Binette L., Magris C. G., Stasińska G., Bruzual A. G., 1994, A\&A, 292, 13

Bottorff M., Lamothe J., Momjian E., Verner E., Vinković D., Ferland G., 1998, PASP, 110, 1040

Bressan A., Marigo P., Girardi L., Salasnich B., Dal Cero C., Rubele S., Nanni A., 2012, MNRAS, 427, 127

Brorby M., Kaaret P., Prestwich A., Mirabel I. F., 2016, MNRAS, 457, 4081

Bruzual G., Charlot S., 2003, MNRAS, 344, 1000

Byler N., Dalcanton J. J., Conroy C., Johnson B. D., Levesque E. M., Berg D. A., 2018, ApJ, 863, 14

Caffau E., Ludwig H.-G., Steffen M., Freytag B., Bonifacio P., 2011, Sol. Phys., 268, 255

Chabrier G., 2003, PASP, 115, 763

Charlot S., Fall S. M., 1993, ApJ, 415, 580

Charlot S., Fall S. M., 2000, ApJ, 539, 718

Charlot S., Longhetti M., 2001, MNRAS, 323, 887

Chen Y., Bressan A., Girardi L., Marigo P., Kong X., Lanza A., 2015, MNRAS, 452, 1068

Chevallard J., Charlot S., 2016, MNRAS, 462, 1415

Chevallard J., et al., 2018, MNRAS, 479, 3264
Chisholm J., Orlitová I., Schaerer D., Verhamme A., Worseck G., Izotov Y. I., Thuan T. X., Guseva N. G., 2017, A\&A, 605, A67

Christensen L., et al., 2012, MNRAS, 427, 1953

Conroy C., Kratter K. M., 2012, ApJ, 755, 123

Crowther P. A., 2006, in Lamers H. J. G. L. M., Langer N., Nugis T., Annuk K., eds, Astronomical Society of the Pacific Conference Series Vol. 353, Stellar Evolution at Low Metallicity: Mass Loss, Explosions, Cosmology. p. 157 (arXiv:astro-ph/0510063)

Crowther P. A., et al., 2016, MNRAS, 458, 624

D’Agostino J. J., Kewley L. J., Groves B., Byler N., Sutherland R. S., Nicholls D., Leitherer C., Stanway E. R., 2019, arXiv e-prints, p. arXiv: 1905.09528

Diaz A. I., Prieto M. A., Wamsteker W., 1988, A\&A, 195, 53

Dors O. L., Cardaci M. V., Hägele G. F., Krabbe Â. C., 2014, MNRAS, 443,1291

Eldridge J. J., Stanway E. R., Xiao L., McClelland L. A. S., Taylor G., Ng M., Greis S. M. L., Bray J. C., 2017, Publ. Astron. Soc. Australia, 34, e058

Erb D. K., Pettini M., Shapley A. E., Steidel C. C., Law D. R., Reddy N. A., 2010, ApJ, 719, 1168

Feltre A., Charlot S., Gutkin J., 2016, MNRAS, 456, 3354

Ferland G. J., et al., 2017, Rev. Mex. Astron. Astrofis., 53, 385

Fosbury R. A. E., et al., 2003, ApJ, 596, 797

Fragos T., et al., 2013, ApJ, 764, 41

Garnett D. R., Kennicutt Jr. R. C., Chu Y.-H., Skillman E. D., 1991, ApJ, 373,458

Giammanco C., Beckman J. E., Cedrés B., 2005, A\&A, 438, 599

Giavalisco M., Koratkar A., Calzetti D., 1996, ApJ, 466, 831

Gilfanov M., Merloni A., 2014, Space Sci. Rev., 183, 121

Gräfener G., Vink J. S., 2015, A\&A, 578, L2

Gräfener G., Koesterke L., Hamann W.-R., 2002, A\&A, 387, 244

Grevesse N., Sauval A. J., 1998, Space Sci. Rev., 85, 161

Groves B. A., Dopita M. A., Sutherland R. S., 2004, ApJS, 153, 75

Gutkin J., Charlot S., Bruzual G., 2016, Monthly Notices of the Royal Astronomical Society, 462, 1757

Hainich R., et al., 2014, A\&A, 565, A27

Hainich R., Pasemann D., Todt H., Shenar T., Sand er A., Hamann W. R., 2015, A\&A, 581, A21

Hamann W.-R., Gräfener G., 2003, A\&A, 410, 993

Hamann W.-R., Gräfener G., 2004, A\&A, 427, 697

Heap S. R., Hubeny I., Lanz T. M., 2001, Astrophysics and Space Science Supplement, 277, 263

Heckman T. M., et al., 2011, ApJ, 730, 5

Hirschmann M., Charlot S., Feltre A., Naab T., Choi E., Ostriker J. P., Somerville R. S., 2017, MNRAS, 472, 2468

Hirschmann M., Charlot S., Feltre A., Naab T., Somerville R. S., Choi E., 2019, MNRAS, 487, 333

Holden B. P., et al., 2016, ApJ, 820, 73

Hubeny I., Lanz T., 2011, Synspec: General Spectrum Synthesis Program, Astrophysics Source Code Library (ascl:1109.022)

Hubeny I., Heap S. R., Lanz T. M., 2000, in American Astronomical Society Meeting Abstracts \#196. p. 718

Izotov Y. I., Thuan T. X., 1998, ApJ, 500, 188

Izotov Y. I., Thuan T. X., Privon G., 2012, MNRAS, 427, 1229

Izotov Y. I., Schaerer D., Thuan T. X., Worseck G., Guseva N. G., Orlitová I., Verhamme A., 2016a, MNRAS, 461, 3683

Izotov Y. I., Orlitová I., Schaerer D., Thuan T. X., Verhamme A., Guseva N. G., Worseck G., 2016b, Nature, 529, 178

Izotov Y. I., Thuan T. X., Guseva N. G., 2017, MNRAS, 471, 548

Izotov Y. I., Schaerer D., Worseck G., Guseva N. G., Thuan T. X., Verhamme A., Orlitová I., Fricke K. J., 2018a, MNRAS, 474, 4514

Izotov Y. I., Worseck G., Schaerer D., Guseva N. G., Thuan T. X., Fricke Verhamme A., Orlitová I., 2018b, MNRAS, 478, 4851

James B. L., Auger M., Pettini M., Stark D. P., Belokurov V., Carniani S., 2018, MNRAS, 476, 1726

Jaskot A. E., Oey M. S., 2013, ApJ, 766, 91

Jaskot A. E., Ravindranath S., 2016, ApJ, 833, 136

Kauffmann G., et al., 2003, MNRAS, 346, 1055 
Kauffmann G., Heckman T. M., De Lucia G., Brinchmann J., Charlot S., Tremonti C., White S. D. M., Brinkmann J., 2006, MNRAS, 367, 1394

Kehrig C., Vílchez J. M., Guerrero M. A., Iglesias-Páramo J., Hunt L. K., Duarte-Puertas S., Ramos-Larios G., 2018, MNRAS, 480, 1081

Kewley L. J., Dopita M. A., Sutherland R. S., Heisler C. A., Trevena J., 2001, ApJ, 556, 121

Kraemer S. B., Wu C.-C., Crenshaw D. M., Harrington J. P., 1994, ApJ, 435,171

Kriek M., van Dokkum P. G., Labbé I., Franx M., Illingworth G. D., Marchesini D., Quadri R. F., 2009, ApJ, 700, 221

Laporte N., Nakajima K., Ellis R. S., Zitrin A., Stark D. P., Mainali R., Roberts-Borsani G. W., 2017, ApJ, 851, 40

Le Fèvre O., et al., 2019, A\&A, 625, A51

Leitet E., Bergvall N., Piskunov N., Andersson B.-G., 2011, A\&A, 532, A107

Leitherer C., et al., 1999, ApJS, 123, 3

Leitherer C., Tremonti C. A., Heckman T. M., Calzetti D., 2011, AJ, 141, 37

Leitherer C., Ekström S., Meynet G., Schaerer D., Agienko K. B., Levesque E. M., 2014, ApJS, 212, 14

Levesque E. M., Leitherer C., 2013, ApJ, 779, 170

Lilly S. J., et al., 2007, ApJS, 172, 70

López-Sánchez Á. R., Esteban C., 2008, A\&A, 491, 131

López-Sánchez Á. R., Esteban C., 2010, A\&A, 516, A104

Lusso E., Worseck G., Hennawi J. F., Prochaska J. X., Vignali C., Stern J., O'Meara J. M., 2015, MNRAS, 449, 4204

Madau P., Fragos T., 2017, ApJ, 840, 39

Mainali R., Kollmeier J. A., Stark D. P., Simcoe R. A., Walth G., Newman A. B., Miller D. R., 2017, ApJ, 836, L14

Marks M., Kroupa P., Dabringhausen J., Pawlowski M. S., 2012, MNRAS, 422, 2246

Maseda M. V., et al., 2017, A\&A, 608, A4

McHardy I., Uttley P., Taylor R., Papadakis I., 2006, in Gaskell C. M., McHardy I. M., Peterson B. M., Sergeev S. G., eds, Astronomical Society of the Pacific Conference Series Vol. 360, AGN Variability from X-Rays to Radio Waves. p. 85

Mignoli M., et al., 2019, arXiv e-prints,

Mollá M., García-Vargas M. L., Bressan A., 2009, MNRAS, 398, 451

Morales-Luis A. B., Sánchez Almeida J., Aguerri J. A. L., Muñoz-Tuñón C., 2011, ApJ, 743, 77

Murray N., 2011, ApJ, 729, 133

Murray N., Quataert E., Thompson T. A., 2010, ApJ, 709, 191

Naidu R. P., et al., 2017, ApJ, 847, 12

Nakajima K., Ouchi M., 2014, MNRAS, 442, 900

Nakajima K., Ellis R. S., Iwata I., Inoue A. K., Kusakabe H., Ouchi M., Robertson B. E., 2016, ApJ, 831, L9

Nakajima K., et al., 2018, A\&A, 612, A94

Nanayakkara T., et al., 2019, A\&A, 624, A89

Nicholls D. C., Dopita M. A., Sutherland R. S., Jerjen H., Kewley L. J., 2014, ApJ, 790, 75

Oey M. S., Kennicutt Jr. R. C., 1993, ApJ, 411, 137

Osterbrock D. E., Ferland G. J., 2006, Astrophysics of gaseous nebulae and active galactic nuclei

Panuzzo P., Bressan A., Granato G. L., Silva L., Danese L., 2003, A\&A, 409, 99

Patrício V., et al., 2016, MNRAS, 456, 4191

Pellegrini E. W., Oey M. S., Winkler P. F., Points S. D., Smith R. C., Jaskot A. E., Zastrow J., 2012, ApJ, 755, 40

Raiter A., Schaerer D., Fosbury R. A. E., 2010, A\&A, 523, A64

Rigby J. R., Bayliss M. B., Gladders M. D., Sharon K., Wuyts E., Dahle H., Johnson T., Peña-Guerrero M., 2015, ApJ, 814, L6

Sana H., et al., 2012, Science, 337, 444

Sander A., Hamann W.-R., Todt H., 2012, A\&A, 540, A144

Sander A., Shenar T., Hainich R., Gímenez-García A., Todt H., Hamann W. R., 2015, A\&A, 577, A13

Schaerer D., Stasińska G., 1999, A\&A, 345, L17

Schaerer D., Fragos T., Izotov Y. I., 2019, A\&A, 622, L10
Schenker M. A., Ellis R. S., Konidaris N. P., Stark D. P., 2013, ApJ, 777, 67

Schmidt K. B., et al., 2017, ApJ, 839, 17

Schmutz W., Hamann W. R., Wessolowski U., 1989, A\&A, 210, 236

Senchyna P., Stark D. P., 2019, MNRAS, 484, 1270

Senchyna P., et al., 2017, MNRAS, 472, 2608

Senchyna P., Stark D. P., Chevallard J., Charlot S., Jones T., Vidal García A., 2019, arXiv e-prints,

Shirazi M., Brinchmann J., 2012, MNRAS, 421, 1043

Smith L. J., Crowther P. A., Calzetti D., Sidoli F., 2016, ApJ, 823, 38

Spitzer L., 1978, Physical processes in the interstellar medium, doi:10.1002/9783527617722.

Stanway E. R., Eldridge J. J., 2018, MNRAS, 479, 75

Stanway E. R., Eldridge J. J., 2019, A\&A, 621, A105

Stanway E. R., Eldridge J. J., Becker G. D., 2016, MNRAS, 456, 485

Stark D. P., 2016, ARA\&A, 54, 761

Stark D. P., et al., 2014, MNRAS, 445, 3200

Stark D. P., et al., 2015, MNRAS, 454, 1393

Stasińska G., 1980, A\&A, 85, 359

Stasińska G., Izotov Y., Morisset C., Guseva N., 2015, A\&A, 576, A83

Steidel C. C., Strom A. L., Pettini M., Rudie G. C., Reddy N. A., Trainor R. F., 2016, ApJ, 826, 159

Sutherland R. S., Dopita M. A., 2017, ApJS, 229, 34

Tang M., Stark D. P., Chevallard J., Charlot S., 2019, MNRAS, p. 2159

Thuan T. X., Izotov Y. I., 2005, ApJS, 161, 240

Todt H., Sander A., Hainich R., Hamann W. R., Quade M., Shenar T., 2015, A\&A, 579, A75

Vanzella E., et al., 2016, ApJ, 821, L27

Vanzella E., et al., 2017, ApJ, 842, 47

Vanzella E., et al., 2019, arXiv e-prints,

Vidal-García A., Charlot S., Bruzual G., Hubeny I., 2017, MNRAS, 470, 3532

Vink J. S., de Koter A., Lamers H. J. G. L. M., 2001, A\&A, 369, 574

Vink J. S., Muijres L. E., Anthonisse B., de Koter A., Gräfener G., Langer N., 2011, A\&A, 531, A132

Xiao L., Stanway E. R., Eldridge J. J., 2018, MNRAS, 477, 904

Yates R. M., Kauffmann G., Guo Q., 2012, MNRAS, 422, 215

Zackrisson E., Inoue A. K., Jensen H., 2013, ApJ, 777, 39

Zackrisson E., et al., 2017, ApJ, 836, 78

Zheng W., Kriss G. A., Telfer R. C., Grimes J. P., Davidsen A. F., 1997, ApJ, 475, 469

de Barros S., et al., 2016, A\&A, 585, A51

\section{APPENDIX A: LIBRARY OF WR-STAR SPECTRA}

The C\&B stellar population model used in this paper incorporates stellar evolutionary tracks computed with the PARSEC code of Bressan et al. (2012) for stars with initial masses up to $600 \mathrm{M}_{\odot}$, which include the evolution through the WR phase (Chen et al. 2015, see also Gutkin et al. 2016). To compute the emission from WR stars, we appeal to the library of high-resolution synthetic spectra from the Potsdam Wolf-Rayet (PoWR) group (Gräfener et al. 2002; Hamann \& Gräfener 2003, 2004; Sander et al. 2012; Hainich et al. 2014, 2015; Sander et al. 2015; Todt et al. 2015). These line-blanketed, non-LTE, spherically expanding models are available for WNE-, WNL-, WC- and WO-type stars in various ranges of stellar effective temperatures, $T_{\star}$, and 'transformed radii', $R_{\mathrm{t}}$, at 4 metallicities, $Z \approx 0.001,0.003,0.006$ and 0.014 (see Table 1). The transformed radius is a convenient, luminosityindependent parametrization of the stellar wind in terms of the stellar radius, $R_{\star}$, terminal wind velocity, $v_{\infty}$, mass-loss rate, $\dot{M}$, and 'density contrast', $D$ (defined as the factor by which the density in the clumps of an inhomogeneous wind is enhanced 
Table 1. PoWR models used in the present paper. $^{a}$

\begin{tabular}{llccccl}
\hline Metallicity & Type & $\log \left(T_{\star} / \mathrm{K}\right)^{b}$ & $\log \left(R_{\mathrm{t}} / \mathrm{R}_{\odot}\right)^{c}$ & $v_{\infty} / \mathrm{km} \mathrm{s}^{-1}$ & $D$ & PARSEC-track metallicity \\
\hline Galaxy & WNL-H20 & {$[4.40,5.00]$} & {$[0.0,1.7]$} & 1000 & 4 & \\
$(Z \approx 0.014)$ & WNE & {$[4.50,5.30]$} & {$[0.0,1.7]$} & 1600 & 4 & $Z \geq 0.014$ \\
& WC & {$[4.60,5.30]$} & {$[-0.5,+1.6]$} & 2000 & 10 & \\
& WO & 5.30 & 0.3 & 1000 & 4 & \\
\hline LMC & WNL-H20 & {$[4.40,5.05]$} & {$[0.0,1.9]$} & 1000 & 10 & \\
$(Z \approx 0.006)$ & WNE & {$[4.60,5.25]$} & {$[-0.2,+1.9]$} & 1600 & 10 & $0.006 \leq Z \leq 0.010$ \\
& WC & {$[4.65,5.30]$} & {$[-0.8,+1.6]$} & 2000 & 10 & \\
\hline SMC & WNL-H20 & {$[4.60,5.05]$} & {$[0.6,1.7]$} & 1600 & 10 & \\
$(Z \approx 0.003)$ & WNE & {$[4.60,5.15]$} & {$[0.2,1.7]$} & 1600 & 10 & $0.002 \leq Z \leq 0.004$ \\
\hline SubSMC & WNL-H20 & {$[4.50,5.05]$} & {$[0.6,1.9]$} & 1600 & 10 & \\
$(Z \approx 0.001)$ & WNE & {$[4.50,5.10]$} & {$[0.0,1.9]$} & 1600 & 10 & $Z \leq 0.001$ \\
\hline
\end{tabular}

${ }^{a}$ Available from http://www.astro.physik.uni-potsdam.de/ PoWR.

${ }^{b}$ Models available in steps of $\Delta\left(\log T_{\star}\right)=0.05$ over the quoted range. The effective temperature $T_{\star}$ is defined at the stellar radius $R_{\star}$ (corresponding to a radial Rosseland continuum optical depth of 20).

${ }^{c}$ Models available in steps of $\Delta\left(\log R_{\mathrm{t}}\right)=0.10$ over the quoted range.

${ }^{d}$ Adopted for all WNL stars in the models (conservatively identified as stars with $T_{\star}>25,000 \mathrm{~K}$ and $\mathrm{H}$-surface abundance by mass $X<0.3$ at all metallicities in the PARSEC tracks).

relative to that of a homogeneous wind of same $\dot{M}$ ), through the formula

$R_{\mathrm{t}}=R_{\star}\left(\frac{v_{\infty}}{2500 \mathrm{~km} \mathrm{~s}^{-1}} / \frac{\dot{M} \sqrt{D}}{10^{-4} \mathrm{M}_{\odot} \mathrm{yr}^{-1}}\right)^{2 / 3}$.

At fixed $R_{\mathrm{t}}$, models with same chemical composition and effective temperature $T_{\star}$ have similar emission-line equivalent widths (see, e.g., Schmutz et al. 1989; Hamann \& Gräfener 2004).

In the C\&B model, the quantities $R_{\star}, T_{\star}$ and $\dot{M}$ for a WR star of metallicity $Z$ are taken from the PARSEC tracks, and the spectrum assigned to that star is taken to be the PoWR model with closest $T_{\star}, \dot{M}$ and $Z$, as indicated in Table 1 (rightmost column). Each PoWR model consists of a high-resolution spectrum (resolving power of $\sim 10,000$ ) over the wavelength range from $200 \AA$ to $8 \mu \mathrm{m}$ and a low-resolution spectrum (resolving power $\lesssim 300$ ) covering from 5 to $200 \AA$. The rates of HI-, He Iand He II-ionizing photons (with wavelengths $\lambda \leq 911.33,504.3$ and $227.85 \AA$, respectively) integrated from the combined spectra do not always match the reference values (computed in an independent way) tabulated for each model on the PoWR website, because of numerical errors in the spectra. In particular, for He II-ionizing photons, whose rate is typically several orders of magnitude lower than those of $\mathrm{He} \mathrm{I}-$ and $\mathrm{HI}$-ionizing photons, the tabulated reference value can often be zero, while spectral integration gives non-zero values. Setting the He II-ionizing flux to zero in the corresponding model spectra, the agreement between integrated and tabulated values for all other spectra used in the $\mathrm{C} \& \mathrm{~B}$ models is always better than 10 per cent. 